\title{
Approximations of Shape Metrics and Application to Shape Warping and Empirical Shape Statistics
}

\author{
Guillaume Charpiat, ${ }^{1}$ Olivier Faugeras, ${ }^{2}$ and Renaud Keriven ${ }^{1,3}$ \\ ${ }^{1}$ Odyssée Laboratory \\ ENS \\ 45 rue d'Ulm \\ 75005 Paris, France \\ guillaume.charpiat@ens.fr \\ ${ }^{2}$ Odyssée Laboratory \\ INRIA Sophia Antipolis \\ 2004 route des Lucioles, BP 93 \\ 06902 Sophia-Antipolis Cedex, France \\ faugeras@sophia.inria.fr \\ ${ }^{3}$ Odyssée Laboratory \\ ENPC \\ 6 av Blaise Pascal \\ 77455 Marne la Vallée, France \\ renaud.keriven@ens.fr
}

\begin{abstract}
This paper proposes a framework for dealing with several problems related to the analysis of shapes. Two related such problems are the definition of the relevant set of shapes and that of defining a metric on it. Following a recent research monograph by Delfour and Zolésio [11], we consider the characteristic functions of the subsets of $\mathbb{R}^{2}$ and their distance functions. The $L^{2}$ norm of the difference of characteristic functions, the $L^{\infty}$ and the $W^{1,2}$ norms of the difference of distance functions define interesting topologies, in particular the well-known Hausdorff distance. Because of practical considerations arising from the fact that we deal with
\end{abstract}

Date received: May 7, 2003. Final version received: March 18, 2004. Date accepted: March 24, 2004. Communicated by Peter Olver. Online publication: July 6, 2004.

AMS classification: 35Q80, 49Q10, 60D05, 62P30, $68 \mathrm{~T} 45$.

Key words and phrases: Shape metrics, Characteristic functions, Distance functions, Deformation flows, Lower semicontinuous envelope, Shape warping, Empirical mean shape, Empirical covariance operator, Principal modes of variation. 


\begin{abstract}
image shapes defined on finite grids of pixels, we restrict our attention to subsets of $\mathbb{R}^{2}$ of positive reach in the sense of Federer [16], with smooth boundaries of bounded curvature. For this particular set of shapes we show that the three previous topologies are equivalent. The next problem we consider is that of warping a shape onto another by infinitesimal gradient descent, minimizing the corresponding distance. Because the distance function involves an inf, it is not differentiable with respect to the shape. We propose a family of smooth approximations of the distance function which are continuous with respect to the Hausdorff topology, and hence with respect to the other two topologies. We compute the corresponding Gâteaux derivatives. They define deformation flows that can be used to warp a shape onto another by solving an initial value problem. We show several examples of this warping and prove properties of our approximations that relate to the existence of local minima. We then use this tool to produce computational definitions of the empirical mean and covariance of a set of shape examples. They yield an analog of the notion of principal modes of variation. We illustrate them on a variety of examples.
\end{abstract}

\title{
1. Introduction
}

Learning shape models from examples, using them to recognize new instances of the same class of shapes, are fascinating problems that have attracted the attention of many scientists for many years. Central to this problem is the notion of a random shape which in itself has occupied researchers for decades. Fréchet [19] is probably one of the first mathematicians to develop some interest in the analysis of random shapes, i.e., curves. He was followed by Matheron [33] who founded, with Serra, the French School of Mathematical Morphology and by D. Kendall [24], [26], [27] and his colleagues, e.g., Small [42]. In addition, and independently, a rich body of theory and practice for the statistical analysis of shapes has been developed by Bookstein [4], Dryden and Mardia [13], Carne [5], and Cootes et al. [8]. Except for the mostly theoretical work of Fréchet and Matheron, the tools developed by these authors are very much tied to the point-wise representation of the shapes they study: objects are represented by a finite number of salient points or landmarks. This is an important difference with our work which deals explicitly with curves as such, independently of their sampling or even parametrization.

In effect, our work bears more resemblance to that of several other authors. As in Grenander's theory of patterns [21], [22], we consider shapes as points of an infinite-dimensional manifold, but we do not model the variations of the shapes by the action of Lie groups on this manifold, except in the case of such finite-dimensional Lie groups as rigid displacements (translation and rotation) or affine transformations (including scaling). For infinite-dimensional groups, such as diffeomorphisms [14], [49] which smoothly change the objects' shapes, previous authors have been dependent upon the choice of parametrizations and origins of coordinates [50], [51], [48], [47], [34], [23]. For them, warping a shape onto another requires the construction of families of diffeomorphisms that use these parametrizations. Our approach, based upon the use of the distance functions, does 
not require the arbitrary choice of parametrizations and origins. From our viewpoint this is already very suitable in two dimensions but becomes even more suitable in three dimensions and higher, where finding parametrizations and tracking origins of coordinates can be a real problem: this is not required in our case. Another piece of related work is that of Soatto and Yezzi [43] who tackle the problem of jointly extracting and characterizing the motion of a shape and its deformation. In order to do this they find inspiration in the above work on the use of diffeomorphisms and propose the use of a distance between shapes (based on the set-symmetric difference described in Section 2.2). This distance poses a number of problems that we address in the same section, where we propose two other distances which we believe to be more suitable. They also use a signed distance score but it is nonsymmetric with respect to the two regions and is not an approximation to a distance.

Some of these authors have also tried to build a Riemannian structure on the set of shapes, i.e., to go from an infinitesimal metric structure to a global one. The infinitesimal structure is defined by an inner product in the tangent space (the set of normal deformation fields) and has to vary continuously from point to point, i.e., from shape to shape. The Riemannian metric is then used to compute geodesic curves between two shapes: these geodesics define a way of warping either shape onto the other. This is dealt with in the work of Trouvé and Younes [50], [51], [49], [48], [47], [52] and, more recently, in the work of Klassen et al. [29], again at the cost of working with parametrizations. The problem with these approaches, beside that of having to deal with parametrizations of the shapes, is that there exist global metric structures on the set of shapes (see Section 2.2) which are useful and relevant to the problem of the comparison of shapes but that do not derive from an infinitesimal structure. Our approach can be seen as taking the problem from exactly the opposite viewpoint from the previous one: we start with a global metric on the set of shapes and build smooth functions (in effect smooth approximations of these metrics) that are dissimilarity measures, or energy functions; we then minimize these functions using techniques of the calculus of variation by computing their gradient and performing infinitesimal gradient descent: this minimization defines another way of warping either shape onto the other. In this endeavor we build on the seminal work of Delfour and Zolésio who have introduced new families of sets, complete metric topologies, and compactness theorems. This work is now available in book form [11]. The book provides a fairly broad coverage and a synthetic treatment of the field along with many new important results, examples, and constructions which have not been published elsewhere. Its full impact on image processing and robotics has yet to be fully assessed.

In this paper we also revisit the problem of computing empirical statistics on sets of two-dimensional shapes and propose a new approach by combining several notions such as topologies on sets of shapes, calculus of variations, and some measure theory. Section 2 sets the stage and introduces some notations and tools. In particular, in Section 2.2 we discuss three of the main topologies that can be defined on sets of shapes and motivate the choice of two of them. In Section 3 
we introduce the particular set of shapes we work with in this paper, show that it has nice compactness properties and that the three topologies defined in the previous section are in fact equivalent on this set of shapes. In Section 4 we introduce one of the basic tools we use for computing shape statistics, i.e., given a measure of the dissimilarity between two shapes, the curve gradient flow that is used to deform a shape into another. Having motivated the introduction of the measures of dissimilarity, we proceed in Section 5 with the construction of classes of such measures which are based on the idea of approximating some of the shape distances that have been presented in Section 2.2; we also prove the continuity of our approximations with respect to these distances and compute the corresponding curve gradient flows. This being settled, we are in a position to warp any given shape onto another by solving the partial differential equation (PDE) attached to the particular curve gradient flow. This problem is studied in Section 6 where examples are also presented. In Section 7.1 we use all these tools to define a meanshape and to provide algorithms for computing it from sample shape examples. In Section 7.2 we extend the notion of the covariance matrix of a set of samples to that of a covariance operator of a set of sample shape examples from which the notion of principal modes of variation follows naturally. We discuss some details of our implementation of these algorithms in Section 8 and conclude in Section 9. This paper is a bit expository in nature in order to make it self-contained. The reader who is familiar with shape topologies can skip Section 2 and go straight to Section 3.1 to read about the definition of our set of shapes from where he/she can jump to Section 4 and the following sections that contain the core of our contributions.

\section{Shapes and Shape Topologies}

To define fully the notion of a shape is beyond the scope of this paper in which we use a limited, i.e., purely geometric, definition. It could be argued that the perceptual shape of an object also depends upon the distribution of illumination, the reflectance and texture of its surface; these aspects are not discussed in this paper. In our context we define a shape to be a measurable subset of $\mathbb{R}^{2}$. Since we are driven by image applications we also assume that all our shapes are contained in a hold-all open bounded subset of $\mathbb{R}^{2}$ which we denote by $D$. The reader can think of $D$ as the "image."

In the next section we will restrict our interest to a more limited set of shapes but, presently, this is sufficient to allow us to introduce some methods for representing shapes.

\subsection{Definitions}

Since, as mentioned in the Introduction, we want to be independent of any particular parametrization of the shape, we use two main ingredients, the characteristic 
function of a shape $\Omega$,

$$
\chi_{\Omega}(x)=1 \quad \text { if } \quad x \in \Omega \quad \text { and } \quad 0 \quad \text { if } \quad x \notin \Omega,
$$

and the distance function to a shape $\Omega$,

$d_{\Omega}(x)=\inf _{y \in \Omega}|y-x|=\inf _{y \in \Omega} d(x, y) \quad$ if $\quad \Omega \neq \emptyset \quad$ and $\quad+\infty \quad$ if $\quad \Omega=\emptyset$.

Note the important property [11, Chap. 4, Theorem 2.1]:

$$
d_{\Omega_{1}}=d_{\Omega_{2}} \quad \Leftrightarrow \quad \bar{\Omega}_{1}=\bar{\Omega}_{2} .
$$

Also of interest is the distance function to the complement of the shape, $d_{\mathrm{C} \Omega}$, and the distance function to its boundary, $d_{\partial \Omega}$. In the case where $\Omega=\partial \Omega$ and $\Omega$ is closed, we have

$$
d_{\Omega}=d_{\partial \Omega}, \quad d_{\mathrm{C} \Omega}=0 .
$$

We note by $C_{d}(D)$ the set of distance functions of nonempty sets of $D$. Similarly, we note by $C_{d}^{c}(D)$ the set of distance functions to the complements of open subsets of $D$ (for technical reasons, which are irrelevant here, it is sufficient to consider open sets).

Another function of great interest is the oriented distance function $b_{\Omega}$ defined as

$$
b_{\Omega}=d_{\Omega}-d_{\mathrm{C} \Omega} .
$$

Note that for closed sets, such that $\Omega=\partial \Omega$, one has $b_{\Omega}=d_{\Omega}$.

We briefly recall some well-known results about these two functions. The integral of the characteristic function is equal to the measure (area) $m(\Omega)$ of $\Omega$ :

$$
\int_{\Omega} \chi_{\Omega}(x) d x=m(\Omega) .
$$

Note that this integral does not change if we add to or subtract from $\Omega$ a measurable set of Lebesgue measure 0 (also called a negligible set).

Concerning the distance functions, they are continuous, in effect, Lipschitz continuous with a Lipschitz constant equal to 1 [9], [11]:

$$
\left|d_{\Omega}(x)-d_{\Omega}(y)\right| \leq|x-y|, \quad \text { for all } x, y \in D .
$$

Thanks to the Rademacher theorem [15], this implies that $d_{\Omega}$ is differentiable almost everywhere (a.e.) in $D$, i.e., outside of a negligible set, and that the magnitude of its gradient, when it exists, is less than or equal to 1 ,

$$
\left|\nabla d_{\Omega}(x)\right| \leq 1 \quad \text { a.e. }
$$

The same is true of $d_{\mathrm{C} \Omega}$ and $b_{\Omega}$ (if $\partial \Omega \neq \emptyset$ for the second) [11, Chap. 5, Theorem 2.1].

Closely related to the various distance functions (more precisely to their gradients) are the projections associated with $\bar{\Omega}$ and $\overline{\mathrm{C} \Omega}$. These are also related to the notion of skeleton. We recall some definitions. The first one is adapted from [11, Chap. 4, Def. 3.1]. 
Definition 1 (Projections and Skeletons).

- Given $\Omega \subset D, \Omega \neq \emptyset$ (resp., $C \Omega \neq \emptyset$ ), the set of projections of $x \in D$ on $\Omega$ (resp., on $C \Omega$ ) is given by

$$
\begin{gathered}
\Pi_{\Omega}(x) \stackrel{\text { def }}{=}\left\{p \in \bar{\Omega}:|p-x|=d_{\Omega}(x)\right\}, \\
\text { (resp., } \left.\Pi_{\mathrm{C} \Omega}(x) \stackrel{\text { def }}{=}\left\{p \in \overline{\mathrm{C} \Omega}:|p-x|=d_{\mathrm{C} \Omega}(x)\right\}\right) .
\end{gathered}
$$

The elements of $\Pi_{\Omega}(x)$ (resp., $\left.\Pi_{\mathrm{C} \Omega}(x)\right)$ are called projections onto $\bar{\Omega}$ (resp., $\overline{\mathrm{C} \Omega})$.

- Given $\Omega \subset D, \Omega \neq \emptyset$ (resp., $\mathrm{C} \Omega \neq \emptyset$ ), the set of points where the projection on $\Omega$ (resp., $C \Omega$ ) is not unique is called the exterior (resp., interior) skeleton $\operatorname{Sk}_{\text {ext }}(\Omega)\left(\right.$ resp., $\operatorname{Sk}_{\text {int }}(\Omega)$ ). We define $\operatorname{Sk}(\Omega)=\operatorname{Sk}_{\text {ext }}(\Omega) \cup \operatorname{Sk}_{\text {int }}(\Omega)$.

The following properties of the skeletons can be found, e.g., in [11, Chap. 4, Theorems 3.1 and 3.2]:

Proposition 2. The exterior (resp., interior) skeleton is exactly the subset of $\mathrm{C} \bar{\Omega}$ (resp., of $\operatorname{int}(\Omega)$ ) where the function $d_{\Omega}\left(r e s p ., d_{\mathrm{C} \Omega}\right)$ is not differentiable. Moreover, the exterior and interior skeletons and the boundary $\partial \Omega$ is exactly the subset of $D$ where $d_{\partial \Omega}$ is not differentiable.

At each $x$ of $C \Omega \backslash \operatorname{Sk}_{\text {ext }}(\Omega)$, the gradient of the distance function $d_{\partial \Omega}$ is welldefined, of unit norm, and points away from the projection $y=\Pi_{\Omega}(x)$ of $x$ onto $\Omega$, see Figure 1. Similar considerations apply to the case where $x \in \Omega$.

We introduce an additional definition that will be useful in the sequel.

Definition 3. Given $\Omega \subset D, \Omega \neq \emptyset$, and a real number $h>0$, the $h$-tubular neighborhood of $\Omega$ is defined as

$$
U_{h}(\Omega) \stackrel{\text { def }}{=}\left\{y \in D: d_{\Omega}(y)<h\right\} .
$$

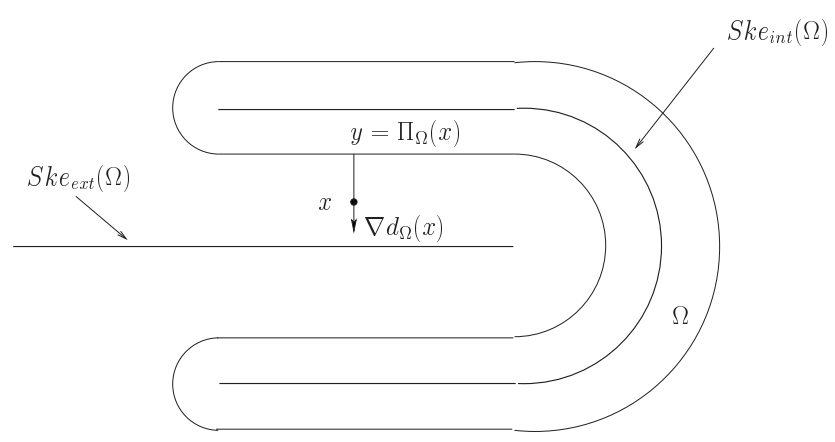

Fig. 1. An example of skeletons. 



$$
\Omega_{1}=\Omega_{2} \backslash\left\{C_{1}, C_{2}, C_{3}\right\}
$$

Fig. 2. Two shapes whose distance $\rho_{2}$ is equal to $0 ; \Omega_{1}$ is obtained by removing, from the disk $\Omega_{2}$, the three curves $C_{1}, C_{2}, C_{3}: \rho_{2}\left(\Omega_{1}, \Omega_{2}\right)=0$.

\subsection{Some Shape Topologies}

The next question we want to address is that of the definition of the similarity between two shapes. This question of similarity is closely connected to that of metrics on sets of shapes which in turn touches that of what is known as shape topologies. We now briefly review three main similarity measures between shapes which turn out to define three distances.

2.2.1. Characteristic Functions. The similarity measure we are about to define is based upon the characteristic functions of the two shapes we want to compare. We denote by $X(D)$ the set of characteristic functions of measurable subsets of $D$.

Given two such sets $\Omega_{1}$ and $\Omega_{2}$, we define their distance

$$
\rho_{2}\left(\Omega_{1}, \Omega_{2}\right)=\left\|\chi_{\Omega_{1}}-\chi_{\Omega_{2}}\right\|_{L^{2}}=\left(\int_{D}\left(\chi_{\Omega_{1}}(x)-\chi_{\Omega_{2}}(x)\right)^{2} d x\right)^{1 / 2} .
$$

This definition also shows that this measure does not "see" differences between two shapes that are of measure 0 (see Figure 2 adapted from [11, Chap. 3, Fig. 3.1]), since the integral does not change if we modify the values of $\chi_{\Omega_{1}}$ or $\chi_{\Omega_{2}}$ over negligible sets. In other words, this is not a distance between the two shapes $\Omega_{1}$ and $\Omega_{2}$ but between their equivalence classes $\left[\Omega_{1}\right]_{m}$ and $\left[\Omega_{2}\right]_{m}$ of measurable sets. Given a measurable subset $\Omega$ of $D$, we define its equivalence class $[\Omega]_{m}$ as $[\Omega]_{m}=\left\{\Omega^{\prime} \mid \Omega^{\prime}\right.$ is measurable and $\Omega \Delta \Omega^{\prime}$ is negligible $\}$, where $\Omega \Delta \Omega^{\prime}$ is the symmetric difference

$$
\Omega \Delta \Omega^{\prime}=\mathrm{C}_{\Omega} \Omega^{\prime} \cup \mathrm{C}_{\Omega^{\prime}} \Omega .
$$

The proof that this defines a distance follows from the fact that the $L^{2}$ norm defines a distance over the set of equivalence classes of square integrable functions (see, e.g., [39], [15]).

This is nice and one has even more [11, Chap. 3, Theorem 2.1]: the set $X(D)$ is closed and bounded in $L^{2}(D)$, and $\rho_{2}(\cdot, \cdot)$ defines a complete metric structure on 
the set of equivalence classes of measurable subsets of $D$. Note that $\rho_{2}$ is closely related to the symmetric difference

$$
\rho_{2}\left(\Omega_{1}, \Omega_{2}\right)=m\left(\Omega_{1} \Delta \Omega_{2}\right)^{1 / 2} .
$$

The completeness is important in applications: any Cauchy sequence of characteristic functions $\left\{\chi_{\Omega_{n}}\right\}$ converges for this distance to a characteristic function $\chi_{\Omega}$ of a limit set $\Omega$. Unfortunately, in applications, not all sequences are Cauchy sequences, for example, the minimizing sequences of the energy functions defined in Section 5, and one often requires more, i.e., that any sequence of characteristic functions contains a subsequence that converges to a characteristic function. This stronger property, called compactness, is not satisfied by $X(D)$ (see [11, Chap. 3]).

2.2.2. Distance Functions. We therefore turn our attention toward a different similarity measure which is based upon the distance function to a shape. As in the case of characteristic functions, we define equivalent sets and say that two subsets $\Omega_{1}$ and $\Omega_{2}$ of $D$ are equivalent iff $\bar{\Omega}_{1}=\bar{\Omega}_{2}$. We note by $[\Omega]_{d}$ the corresponding equivalence class of $\Omega$. Let $\mathcal{T}(D)$ be the set of these equivalence classes. The application

$$
[\Omega]_{d} \rightarrow d_{\Omega}: \quad \mathcal{T}(D) \rightarrow C_{d}(D) \subset C(\bar{D})
$$

is injective according to (1). We can therefore identify the set $C_{d}(D)$ of distance functions to sets of $D$ with the just-defined set of equivalence classes of sets.

Since $C_{d}(D)$ is a subset of the set $C(\bar{D})$ of continuous functions on $\bar{D}$, a Banach space $^{1}$ when endowed with the norm

$$
\|f\|_{C(D)}=\sup _{x \in D}|f(x)|,
$$

it can be shown (e.g., [11]) that the similarity measure

$$
\rho\left(\left[\Omega_{1}\right]_{d},\left[\Omega_{2}\right]_{d}\right)=\left\|d_{\Omega_{1}}-d_{\Omega_{2}}\right\|_{C(D)}=\sup _{x \in D}\left|d_{\Omega_{1}}(x)-d_{\Omega_{2}}(x)\right|
$$

is a distance on the set of equivalence classes of sets which induces on this set a complete metric. Moreover, because we have assumed $D$ bounded, the corresponding topology is identical to the one induced by the well-known Hausdorff metric (see [33], [40], [11])

$$
\rho_{H}\left(\left[\Omega_{1}\right]_{d},\left[\Omega_{2}\right]_{d}\right)=\max \left\{\sup _{x \in \Omega_{2}} d_{\Omega_{1}}(x), \sup _{x \in \Omega_{1}} d_{\Omega_{2}}(x)\right\} .
$$

In fact, we have even more than the identity of the two topologies, see [11, Chap. 4, Theorem 2.2].

\footnotetext{
${ }^{1}$ A Banach space is a complete normed vector space.
} 
Proposition 4. If the hold-all set $D$ is bounded $\rho=\rho_{H}$.

An important improvement with respect to the situation in the previous section is (see [11, Chap. 4, Theorem 2.2]):

Theorem 5. The set $C_{d}(D)$ is compact in the set $C(\bar{D})$ for the topology defined by the Hausdorff distance.

In particular, from any sequence $\left\{d_{\Omega_{n}}\right\}$ of distance functions to sets $\Omega_{n}$, one can extract a sequence converging toward the distance function $d_{\Omega}$ to a subset $\Omega$ of $D$.

It would appear that we have reached an interesting stage and that the Hausdorff distance is what we want to measure shape similarities. Unfortunately, this is not so because the convergence of areas and perimeters is lost in the Hausdorff metric, as shown in the following example taken from [11, Chap. 4, Example 4.1 and Fig. 4.3].

Consider the sequence $\left\{\Omega_{n}\right\}$ of sets in the open square $]-1,2\left[^{2}\right.$ :

$$
\Omega_{n}=\left\{(x, y) \in D: \frac{2 k}{2 n} \leq x \leq \frac{2 k+1}{2 n}, 0 \leq k<n\right\} .
$$

Figure 3 shows the sets $\Omega_{4}$ and $\Omega_{8}$. This defines $n$ vertical stripes of equal width $1 / 2 n$ each distant of $1 / 2 n$. It is easy to verify that, for all $n \geq 1, m\left(\Omega_{n}\right)=\frac{1}{2}$ and $\left|\partial \Omega_{n}\right|=2 n+1$. Moreover, if $S$ is the unit square $[0,1]^{2}$, for all $x \in S$, $d_{\Omega_{n}}(x) \leq 1 / 4 n$, hence $d_{\Omega_{n}} \rightarrow d_{S}$ in $C(\bar{D})$. The sequence $\left\{\Omega_{n}\right\}$ converges to $S$ for the Hausdorff distance but since $m\left(\bar{\Omega}_{n}\right)=m\left(\Omega_{n}\right)=\frac{1}{2} \nrightarrow 1=m(S), \chi_{\Omega_{n}} \nrightarrow \chi_{S}$ in $L^{2}(D)$ and hence we do not have convergence for the $\rho_{2}$ topology. Note also that $\left|\partial \Omega_{n}\right|=2 n+1 \nrightarrow|\partial S|=4$.

2.2.3. Distance Functions and Their Gradients. In order to recover continuity of the area one can proceed as follows. If we recall that the gradient of a distance function is of magnitude equal to 1 except on a subset of measure 0 of $D$, one

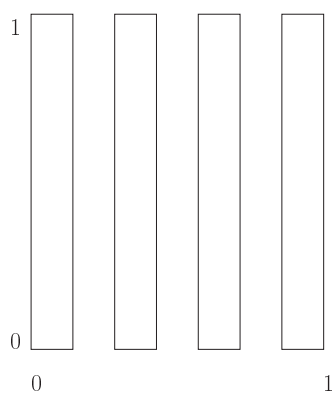

(a)

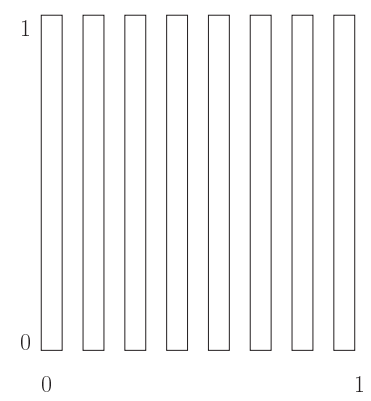

(b)

Fig. 3. Two shapes in the sequence $\left\{\Omega_{n}\right\}$, see text: (a) $\Omega_{4}$ and (b) $\Omega_{8}$. 
concludes that it is square integrable on $D$. Hence the distance functions and their gradients are square-integrable, they belong to the Sobolev space $W^{1,2}(D)$, a Banach space for the vector norm

$$
\|f-g\|_{W^{1,2}(D)}=\|f-g\|_{L^{2}(D)}+\|\nabla f-\nabla g\|_{\mathbf{L}^{2}(D)},
$$

where $\mathbf{L}^{2}(D)=L^{2}(D) \times L^{2}(D)$. This defines a similarity measure for two shapes

$$
\rho_{D}\left(\left[\Omega_{1}\right]_{d},\left[\Omega_{2}\right]_{d}\right)=\left\|d_{\Omega_{1}}-d_{\Omega_{2}}\right\|_{W^{1,2}(D)},
$$

which turns out to define a complete metric structure on $\mathcal{T}(D)$. The corresponding topology is called the $W^{1,2}$-topology. For this metric, the set $C_{d}(D)$ of distance functions is closed in $W^{1,2}(D)$, and the mapping

$$
d_{\Omega} \rightarrow \chi_{\bar{\Omega}}=1-\left|\nabla d_{\Omega}\right|: C_{d}(D) \subset W^{1,2}(D) \rightarrow L^{2}(D)
$$

is "Lipschitz continuous":

$$
\left\|\chi_{\bar{\Omega}_{1}}-\chi_{\bar{\Omega}_{2}}\right\|_{L^{2}(D)} \leq\left\|\nabla d_{\Omega_{1}}-\nabla d_{\Omega_{2}}\right\|_{\mathbf{L}^{2}(D)} \leq\left\|d_{\Omega_{1}}-d_{\Omega_{2}}\right\|_{W^{1,2}(D)},
$$

which indeed shows that areas are continuous for the $W^{1,2}$-topology, see [11, Chap. 4, Theorem 4.1].

$C_{d}(D)$ is not compact for this topology but a subset of it of great practical interest is, see Section 3.

\section{The Set $\mathcal{S}$ of All Shapes and its Properties}

We now have all the necessary ingredients to be more precise in the definition of shapes.

\subsection{The Set of All Shapes}

We restrict ourselves to sets of $D$ with compact boundary and consider three different sets of shapes. The first one is adapted from [11, Chap. 4, Def. 5.1]:

Definition 6 (Set $\mathcal{D Z}$ of Sets of Bounded Curvature). The set $\mathcal{D Z}$ of sets of bounded curvature contains those subsets $\Omega$ of $\bar{D}, \Omega, \mathrm{C} \Omega \neq \emptyset$ such that $\nabla d_{\Omega}$ and $\nabla d_{\mathrm{C} \Omega}$ are in $\mathrm{BV}(D)^{2}$, where $\mathrm{BV}(D)$ is the set of functions of bounded variations.

This is a large set (too large for our applications) which we use as a "frame of reference." $\mathcal{D Z}$ was introduced by Delfour and Zolésio [9], [10] and contains the sets $\mathcal{F}$ and $\mathcal{C}_{2}$ introduced below. For technical reasons related to compactness properties (see Section 3.2) we consider the following subset of $\mathcal{D Z}$ : 
Definition 7 (Set $\mathcal{D} \mathcal{Z}_{0}$ ). The set $\mathcal{D} \mathcal{Z}_{0}$ is the subset of $\mathcal{D} \mathcal{Z}$ such that there exists $c_{0}>0$ such that, for all $\Omega \in \mathcal{D} \mathcal{Z}_{0}$,

$$
\left\|D^{2} d_{\Omega}\right\|_{M^{1}(D)} \leq c_{0} \quad \text { and } \quad\left\|D^{2} d_{\mathrm{C} \Omega}\right\|_{M^{1}(D)} \leq c_{0},
$$

where $M^{1}(D)$ is the set of bounded measures on $D$ and $\left\|D^{2} d_{\Omega}\right\|_{M^{1}(D)}$ is defined as follows. Let $\Phi$ be a $2 \times 2$ matrix of functions in $C^{1}(D)$, we have

$$
\left\|D^{2} d_{\Omega}\right\|_{M^{1}(D)}=\sup _{\boldsymbol{\Phi} \in C^{1}(D)^{2 \times 2},\|\boldsymbol{\Phi}\|_{C} \leq 1}\left|\int_{D} \nabla d_{\Omega} \cdot \operatorname{div} \boldsymbol{\Phi} d x\right|,
$$

where

$$
\|\boldsymbol{\Phi}\|_{C}=\sup _{x \in D}|\boldsymbol{\Phi}(x)|_{\mathbb{R}^{2 \times 2}}
$$

and

$$
\operatorname{div} \Phi=\left[\operatorname{div} \Phi_{1}, \operatorname{div} \Phi_{2}\right]
$$

where $\Phi_{i}, i=1,2$, are the row vectors of the matrix $\Phi$.

The set $\mathcal{D} \mathcal{Z}_{0}$ has the following property (see [11, Chap. 4, Theorem 5.2]):

Proposition 8. Any $\Omega \in \mathcal{D} \mathcal{Z}_{0}$ has a finite perimeter upper-bounded by $2 c_{0}$.

We next introduce three related sets of shapes.

Definition 9 (Sets of Smooth Shapes). The set $\mathcal{C}_{0}$ (resp., $\mathcal{C}_{1}, \mathcal{C}_{2}$ ) of smooth shapes is the set of subsets of $D$ whose boundary is nonempty and can be locally represented as the graph of a $C^{0}$ (resp., $\left.C^{1}, C^{2}\right)$ function. One further distinguishes the sets $\mathcal{C}_{i}^{c}$ and $\mathcal{C}_{i}^{o}, i=0,1,2$, of subsets whose boundary is closed and open, ${ }^{2}$ respectively.

Note that this implies that the boundary is a simple regular curve (hence compact) since otherwise it cannot be represented as the graph of a $C^{0}$ (resp., $C^{1}, C^{2}$ ) function in the vicinity of a multiple point. Also note that when $\Omega \in \mathcal{C}_{i}^{o}$, the set is identical to its boundary: $\Omega=\partial \Omega=\Gamma$. Another consequence of this definition is that the shape $\Omega_{1}$ on the left-hand side of Figure 2 is not in $\mathcal{C}_{i}, i=0,1,2$, because the curves $C_{1}, C_{2}, C_{3}$, parts of $\partial \Omega_{1}$, cannot be represented as graphs of a $C^{i}, i=0,1,2$, function (see, e.g., [9, Chap. 2, Def. 3.1]). Also note that $\mathcal{C}_{2} \subset \mathcal{C}_{1} \subset \mathcal{D Z}[\mathbf{9}],[\mathbf{1 0}]$.

The third set has been introduced by Federer [16].

\footnotetext{
${ }^{2}$ Meaning here, without and with endpoints, respectively.
} 
Definition 10 (Set $\mathcal{F}$ of Shapes of Positive Reach). A nonempty subset $\Omega$ of $D$ is said to have positive reach if there exists $h>0$ such that $\Pi_{\Omega}(x)$ is a singleton for every $x \in U_{h}(\Omega)$. The maximum $h$ for which the property holds is called the reach of $\Omega$ and is noted reach $(\Omega)$.

We will also be interested in the subsets, called $h_{0}$-Federer's sets and noted $\mathcal{F}_{h_{0}}, h_{0}>0$, of $\mathcal{F}$ which contain all Federer's sets $\Omega$ such that $\operatorname{reach}(\Omega) \geq h_{0}$. Note that $\mathcal{C}_{i}, i=0,1,2 \subset \mathcal{F}$, but $\mathcal{C}_{i} \not \subset \mathcal{F}_{h_{0}}$.

We are now ready to define the set of shapes of interest.

Definition 11 (Set of All Shapes). The set, noted $\mathcal{S}$, of all shapes (of interest) is the subset of $\mathcal{C}_{2}$ whose elements are also $h_{0}$-Federer's sets for a given and fixed $h_{0}>0$,

$$
\mathcal{S} \stackrel{\text { def }}{=} \mathcal{C}_{2} \cap \mathcal{F}_{h_{0}} .
$$

This set contains the two subsets $\mathcal{S}^{c}$ and $\mathcal{S}^{o}$ obtained by considering $\mathcal{C}_{2}^{c}$ and $\mathcal{C}_{2}^{o}$, respectively.

Note that $\mathcal{S} \subset \mathcal{D Z}$. Note also that the curvature of $\partial \Omega$ is well-defined and upper-bounded by $1 / h_{0}$, noted $\kappa_{0}$. Hence, $c_{0}$ in Definition 7 can be chosen in such a way that $\mathcal{S} \subset \mathcal{D} \mathcal{Z}_{0}$.

At this point, we can represent regular (i.e., $C^{2}$ ) simple curves with and without boundaries that do not curve or pinch too much (in the sense of $\kappa_{0}$ and $h_{0}$, see Figure 4). Polygons and other nonsmooth structures are not explicitly included in our theory which assumes smooth shapes. In practice, they are, thanks to the fact that we intersect $\mathcal{C}_{2}$ with $\mathcal{F}_{h_{0}}$. If $h_{0}$ is chosen to be smaller than the smallest

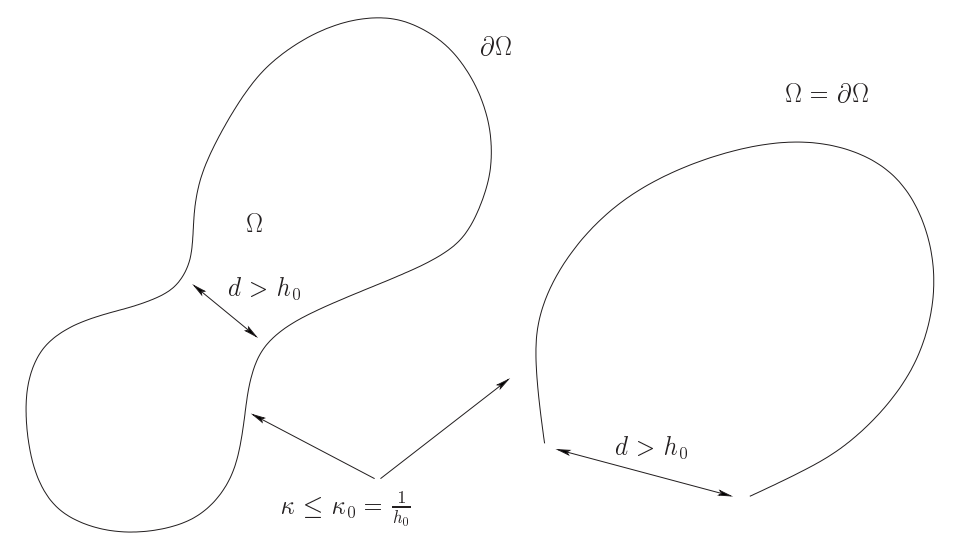

(a)

(b)

Fig. 4. Examples of admissible shapes: (a) a simple, closed, regular curve; (b) a simple, open regular curve. In both cases the curvature is upper-bounded by $\kappa_{0}$ and the pinch distance is larger than $h_{0}$. 
distance between pixels, we will not see the difference between a polygon or a nonsmooth shape and its approximation by an element of $\mathcal{S}$.

There are two reasons why we choose $\mathcal{S}$ as our frame of reference. The first one is because our implementations work with discrete objects defined on an underlying discrete square grid of pixels. As a result we are not able to describe details smaller than the distance between two pixels. This is our unit, our absolute yardstick, and $h_{0}$ is chosen to be smaller than or equal to it. The second reason is that $\mathcal{S}$ is included in $\mathcal{D} \mathcal{Z}_{0}$ which, as shown in Section 3.2, is compact. This will turn out to be important when minimizing shape functionals.

In the remainder of this paper, the distances $\rho\left(\rho_{H}\right)$ and $\rho_{D}$ use the distance functions of the boundaries of the sets we consider.

The question of the deformation of a shape by an element of a group of transformations could be raised at this point. What we have in mind here is the question of deciding whether a square and the same square rotated by 45 degrees are the same shape. There is no real answer to this question, more precisely the answer depends on the application. Note that the group in question can be finite-dimensional, as in the case of the Euclidean and affine groups which are the most common in applications, or infinite-dimensional. In this work we will, for the most part, not consider the action of groups of transformations on shapes.

\subsection{Compactness Properties}

Interestingly enough, the definition of the set $\mathcal{D} \mathcal{Z}_{0}$ (Definition 7) implies that it is compact for all three topologies. This is the result of the following theorem whose proof can be found in [11, Chap. 4, Theorems 8.2, 8.3]:

Theorem 12. Let $D$ be a nonempty bounded regular ${ }^{3}$ open subset of $\mathbb{R}^{2}$ and $\mathcal{D} \mathcal{Z}$ the set defined in Definition 6. The embedding

$$
B C(D)=\left\{d_{\Omega} \in C_{d}(D) \cap C_{d}^{c}(D): \nabla d_{\Omega}, \nabla d_{\mathrm{C} \Omega} \in B V(D)^{2}\right\} \rightarrow W^{1,2}(D),
$$

is compact.

This means that for any bounded sequence $\left\{\Omega_{n}\right\}, \emptyset \neq \Omega_{n}$ of elements of $\mathcal{D Z}$, i.e., for any sequence of $\mathcal{D} \mathcal{Z}_{0}$, there exists a set $\Omega \neq \emptyset$ of $\mathcal{D} \mathcal{Z}$ such that there exists a subsequence $\Omega_{n_{k}}$ with

$$
d_{\Omega_{n_{k}}} \rightarrow d_{\Omega} \quad \text { and } \quad d_{\mathrm{C} \Omega_{n_{k}}} \rightarrow d_{\mathrm{C} \Omega} \quad \text { in } W^{1,2}(D) .
$$

Since $b_{\Omega}=d_{\Omega}-d_{\mathrm{C} \Omega}$, we also have the convergence of $b_{\Omega_{n_{k}}}$ to $b_{\Omega}$, and since the mapping $b_{\Omega} \rightarrow\left|b_{\Omega}\right|=d_{\partial \Omega}$ is continuous in $W^{1,2}(D)$ (see [11, Chap. 5,

\footnotetext{
${ }^{3}$ Regular means uniformly Lipschitzian in the sense of [11, Chap. 2, Def. 5.1].
} 
Theorem 5.1(iv)]), we also have the convergence of $d_{\partial \Omega_{n_{k}}}$ to $d_{\partial \Omega}$. The convergence for the $\rho_{2}$ distance follows from equation (5):

$$
\chi_{\Omega_{n_{k}}} \rightarrow \chi_{\Omega} \text { in } L^{2}(D),
$$

and the convergence for the Hausdorff distance follows from Theorem 5, taking subsequences if necessary.

In other words, the set $\mathcal{D} \mathcal{Z}_{0}$ is compact for the topologies defined by the $\rho_{2}$, Hausdorff, and $W^{1,2}$ distances.

Note that, even though $\mathcal{S} \subset \mathcal{D Z}_{0}$, this does not imply that it is compact for either one of these three topologies. But it does imply that its closure $\overline{\mathcal{S}}$ for each of these topologies is compact in the compact set $\mathcal{D} \mathcal{Z}_{0}$.

\subsection{Comparison Between the Three Topologies on $\mathcal{S}$}

The three topologies we have considered turn out to be closely related on $\mathcal{S}$. This is summarized in the following:

Theorem 13. The three topologies defined by the three distances $\rho_{2}, \rho_{D}$, and $\rho_{H}$ are equivalent on $\mathcal{S}^{c}$. The two topologies defined by $\rho_{D}$ and $\rho_{H}$ are equivalent on $\mathcal{S}^{o}$.

This means that, for example, given a set $\Omega$ of $\mathcal{S}^{c}$, a sequence $\left\{\Omega_{n}\right\}$ of elements of $\mathcal{S}^{c}$ converging toward $\Omega \in \mathcal{S}^{c}$ for any of the three distances $\rho_{2}, \rho\left(\rho_{H}\right)$, and $\rho_{D}$ also converges toward the same $\Omega$ for the other two distances.

We now proceed with the proof of Theorem 13. Being a bit lengthy, we have split it into a series of lemmas and propositions.

We start with a lemma.

Lemma 14. Let $\left\{f_{n}\right\}$ be a sequence of uniformly Lipschitz functions $K \rightarrow \mathbb{R}^{m}$, $K$ a compact of $\mathbb{R}^{2}$, converging for the $L^{2}$ norm toward a Lipschitz continuous function $f$. Then the convergence is uniform.

Proof. The $L^{2}$ convergence of continuous functions implies the convergence a.e. Let us show that this implies the convergence everywhere. We note by $L$ the Lipschitz constant. Let $x_{0}$ be a point of $K$ such that $f_{n}\left(x_{0}\right)$ does not converge toward $f\left(x_{0}\right)$. There exists $\varepsilon_{0}>0$ such that for all $n_{0} \geq 0$, there exists $n>n_{0}$, $\left|f_{n}\left(x_{0}\right)-f\left(x_{0}\right)\right|>\varepsilon_{0}$.

$f$ being continuous at $x_{0}$, there exists $\eta>0$ such that for all $y$ in $K$ such that $d\left(x_{0}, y\right)<\eta,\left|f(y)-f\left(x_{0}\right)\right|<\varepsilon_{0} / 3$.

Consider now the $y$ 's of $K$ such that $d\left(x_{0}, y\right)<\inf \left(\varepsilon_{0} / 3 L, \eta\right)$. There exists at least one of them, noted $y_{0}$, such that $f_{n}\left(y_{0}\right)$ converges to $f\left(y_{0}\right)$ because the convergence is a.e. 
We write

$$
\left|f_{n}\left(x_{0}\right)-f\left(x_{0}\right)\right| \leq\left|f_{n}\left(x_{0}\right)-f_{n}\left(y_{0}\right)\right|+\left|f_{n}\left(y_{0}\right)-f\left(y_{0}\right)\right|+\left|f\left(y_{0}\right)-f\left(x_{0}\right)\right| .
$$

The first term on the right-hand side of this inequality is less than or equal to $\varepsilon_{0} / 3$ because of the uniform Lipschitz hypothesis. Because $f_{n}\left(y_{0}\right)$ converges to $f\left(y_{0}\right)$ there exists $N_{0}\left(\varepsilon_{0}, y\right)$ such that for all $n \geq N_{0},\left|f_{n}\left(y_{0}\right)-f\left(y_{0}\right)\right| \leq \varepsilon_{0} / 3$. The third term is also less than or equal to $\varepsilon_{0} / 3$ because of the hypothesis on $f$. Hence

$$
\left|f_{n}\left(x_{0}\right)-f\left(x_{0}\right)\right| \leq \varepsilon_{0}, \quad \text { for all } n \geq N_{0},
$$

a contradiction. The sequence $\left\{f_{n}\right\}$ converges toward $f$ everywhere in $K$ and since the $f_{n}$ 's are uniformly Lipschitz, the convergence is uniform (see, e.g., [12]).

This lemma is useful for proving the following:

Proposition 15. In $\mathcal{S}$, the $W^{1,2}$ convergence of sequences of distance functions implies their Hausdorff convergence.

Proof. The $W^{1,2}$ convergence implies the $L^{2}$ convergence of the distance functions. According to Lemma 14 this implies the uniform convergence of the distance functions and hence the Hausdorff convergence.

We also have the converse

Proposition 16. In $\mathcal{S}$, the Hausdorff convergence of sequences of distance functions implies their $W^{1,2}$ convergence.

Proof. We consider the boundary $\Gamma$ of a shape $\Omega$ of $\mathcal{S}$. The inequality

$$
\left\|d_{\Gamma_{1}}-d_{\Gamma_{2}}\right\|_{L^{2}} \leq \rho\left(\Gamma_{1}, \Gamma_{2}\right) m(D)^{1 / 2}
$$

shows that the Hausdorff convergence implies the $L^{2}$ convergence of the distance functions. For the $W^{1,2}$ topology we also need the convergence of the $L^{2}$ norm of the gradient.

Consider a sequence $\left\{\Omega_{n}\right\}$ of elements of $\mathcal{S}$ whose boundaries $\Gamma_{n}$ converge for the Hausdorff distance toward $\Gamma \in \mathcal{S}$. If we prove the convergence a.e. of $\nabla\left(d_{\Gamma_{n}}-d_{\Gamma}\right)$ to 0 , the Lebesgue dominated convergence theorem will give us the $L^{2}$ convergence toward 0 since

$$
\left|\nabla\left(d_{\Gamma_{n}}-d_{\Gamma}\right)\right| \leq 2 \text { a.e. }
$$

Because we are in $\mathcal{S}$, all skeletons are negligible (zero Lebesgue measure) [6]. Consider the union $S k=\Gamma \cup S k(\Gamma) \cup_{n} S k\left(\Gamma_{n}\right) \cup_{n} \Gamma_{n}$; as a denumerable union of negligible sets it is negligible. Let $x$ be a point of $D \backslash S k, y_{n}$ its projection on 
$\Gamma_{n}, y$ its projection on $\Gamma$. According to Definition 1 and Proposition 2, all distance functions of interest are differentiable at $x$. We prove that the angle between the vectors $x \vec{y}_{n}$ and $x \vec{y}_{n}$ goes to 0 by proving that $y_{n} \rightarrow y$. By compactness of $\bar{D}$ there exists a subsequence $\left\{y_{n_{k}}\right\}$ of $\left\{y_{n}\right\}$ converging toward $z \in \Gamma$. If $z=y$ we are done. If $z \neq y$ we prove a contradiction. Indeed, since the distance is continuous

$$
\lim _{k \rightarrow \infty} d\left(x, y_{n_{k}}\right)=d(x, y) .
$$

But we also have, by definition, $d\left(x, y_{n_{k}}\right)=d_{\Gamma_{n_{k}}}(x)$; since $\Gamma_{n_{k}} \rightarrow \Gamma$ for the Hausdorff distance, $d_{\Gamma_{n_{k}}} \rightarrow d_{\Gamma}$ everywhere in $D$ and therefore $\lim _{k \rightarrow \infty} d_{\Gamma_{n_{k}}}(x)=$ $d_{\Gamma}(x)$. Hence $d(x, y)=d(x, z)$ and $x \in S k(\Gamma)$, a contradiction.

We have shown that all converging subsequences of $\left\{y_{n}\right\}$ converged to $z=$ $\Pi_{\Gamma}(x)$. In order to conclude, we must show that the sequence $\left\{y_{n}\right\}$ converges to $z$. Indeed, let us assume that there exists a subsequence $\left\{y_{n_{k}}\right\}$ not converging. There exists an $\varepsilon_{0}>0$ such that there is an infinity of values of $k$ for which $y_{n_{k}}$ is outside the open disk $B\left(z, \varepsilon_{0}\right)$. Let us note by $\left\{y_{n_{l}}\right\}$ the corresponding subsequence. Because of compactness again there exists a converging subsequence of $\left\{y_{n_{l}}\right\}$ which has to converge toward $z$ but this is impossible since all $y_{n_{l}}$ are ouside $B\left(z, \varepsilon_{0}\right)$. Hence the sequence $\left\{y_{n}\right\}$ converges toward $z$ and we have proved that $\nabla\left(d_{\Gamma_{n}}-d_{\Gamma}\right) \rightarrow 0$ a.e.

We now compare the topologies induced by the $\rho_{2}$ and the Hausdorff distances. This only makes sense in $\mathcal{S}^{c}$. The first result is the following:

Proposition 17. In $\mathcal{S}^{c}$, the Hausdorff convergence of sequences of distance functions to the boundaries implies the $L^{2}$ convergence of the corresponding characteristic functions of the sets.

Proof. The proof is based on the proof of Proposition 23 below where we show that if $\rho_{H}\left(\Gamma_{1}, \Gamma_{2}\right)<\varepsilon<h_{0}$, given a $C^{2}$ parametrization $p \in[0,1] \rightarrow \Gamma_{1}(p)$ of $\Gamma_{1}$, we can build a $C^{2}$ parametrization $p \in[0,1] \rightarrow \Gamma_{2}(p)$ of $\Gamma_{2}$ such that the vector $\overrightarrow{\Gamma_{1}(p) \Gamma_{2}(p)}$ is normal to $\Gamma_{2}$ for all $p$ 's. Let $s_{2}$ be the arc length on $\Gamma_{2}$, $L_{2}$ its length. The integral $\int_{0}^{L_{2}}\left\|\overrightarrow{\Gamma_{1}\left(s_{2}\right) \Gamma_{2}\left(s_{2}\right)}\right\| d s_{2}$ is equal to $m\left(\Omega_{1} \Delta \Omega_{2}\right)$, hence to $\left(\rho_{2}\left(\Omega_{1}, \Omega_{2}\right)\right)^{2}$ (equation (2)). Since $\left\|\overrightarrow{\Gamma_{1}\left(s_{2}\right) \Gamma_{2}\left(s_{2}\right)}\right\| \leq \max _{p} d_{\Gamma_{2}}\left(\Gamma_{1}(p)\right) \leq$ $\rho_{H}\left(\Gamma_{1}, \Gamma_{2}\right)$, we have $\left(\rho_{2}\left(\Omega_{1}, \Omega_{2}\right)\right)^{2} \leq \varepsilon L_{2} \leq 2 \varepsilon c_{0}$, according to Proposition 8. $\square$

We also prove the converse in

Proposition 18. In $\mathcal{S}^{c}$, the $\rho_{2}$ convergence of sequences of characteristic functions implies the Hausdorff convergence of the distance functions of the boundaries of the corresponding sets.

In the proof we will need the following two lemmas and proposition: 
Lemma 19. Let $\Gamma$ be a $C^{2}$ curve whose curvature is upper-bounded by $\kappa_{0}$. Let $C_{1}$ and $C_{2}$ be two points of $\Gamma, \delta$ the length of the curve between $C_{1}$ and $C_{2}$ :

$$
0 \leq \delta-d\left(C_{1}, C_{2}\right) \leq \frac{\delta^{2} \kappa_{0}}{2}
$$

Proof. The first inequality in (6) follows from the fact that the straight line is the shortest path between two points in the plane.

We parameterize $\Gamma$ with its arc length $s$. We recall the Frenet formulas

$$
\frac{d \Gamma}{d s}=\mathbf{t}, \quad \frac{d \mathbf{t}}{d s}=\kappa \mathbf{n}, \quad \frac{d \mathbf{n}}{d s}=-\kappa \mathbf{t},
$$

where $\mathbf{t}$ and $\mathbf{n}$ are the unit tangent and normal vectors to $\Gamma$, respectively. We then write the second-order Taylor expansion without remainder of $\Gamma\left(s_{2}\right)=C_{2}$ at $\Gamma\left(s_{1}\right)=C_{1}$,

$$
\begin{aligned}
C_{2}= & C_{1}+\left(s_{2}-s_{1}\right) \mathbf{t}\left(s_{1}\right)+\left(s_{2}-s_{1}\right)^{2} \\
& \times \int_{0}^{1}(1-\zeta) \kappa\left(s_{1}+\zeta\left(s_{2}-s_{1}\right)\right) \mathbf{n}\left(s_{1}+\zeta\left(s_{2}-s_{1}\right)\right) d \zeta .
\end{aligned}
$$

The second inequality in (6) follows from the fact that $|\kappa| \leq \kappa_{0}$ and $\delta=\left|s_{2}-s_{1}\right|$.

An easy consequence of this lemma is

Proposition 20. The length of a closed curve in $\mathcal{S}$ is greater than or equal to $2 h_{0}$.

Proof. We use the second inequality in (6) with $d\left(C_{1}, C_{2}\right)=0$ from which the conclusion follows.

The second lemma tells us that in a disk of small enough radius we cannot have too large a piece of a boundary of an element of $\mathcal{S}$.

Lemma 21. Let $\varepsilon>0$ be such that $2 \varepsilon \kappa_{0} \ll 1$. Then any disk of radius $\varepsilon$ does not contain a connected piece of boundary of an element of $\mathcal{S}$ of length greater than $h_{0}$.

Proof. The proof follows from the previous lemma. Let us first assume that the piece in question has a boundary, hence two different endpoints $C_{1}$ and $C_{2}$. By definition, $d\left(C_{1}, C_{2}\right) \leq 2 \varepsilon$. Using (6) we conclude that the length $\delta$ of the curve between $C_{1}$ and $C_{2}$ must satisfy

$$
\frac{\delta^{2} \kappa_{0}}{2}-\delta+2 \varepsilon \geq 0
$$


The left-hand side is a second degree polynomial in the variable $\delta$, noted $P(\delta)$, which has two positive roots $\delta_{1} \leq \delta_{2}$ :

$$
\delta_{1}=h_{0}\left(1-\sqrt{1-2 \varepsilon \kappa_{0}}\right) .
$$

Since $P(0)>0, \delta$ can continuously vary from 0 to its maximal value, and $\delta_{1}<\delta_{2}$, we must have $\delta \leq \delta_{1}$. Moreover, since $2 \varepsilon \kappa_{0} \ll 1, \delta_{1}<h_{0} / 2$.

Let us now assume that the connected piece does not have a boundary, hence is a closed simple curve. We choose two distinct arbitrary points $C_{1}$ and $C_{2}$ on the curve, apply the previous analysis to the each of the two connected components, and conclude that the length of the curve is less than $h_{0}$. Because of Proposition 20, this is impossible.

We now prove Proposition 18.

Proof. Let $\Omega_{1}$ and $\Omega_{2}$ be two shapes of $\mathcal{S}^{c}$ with boundaries $\Gamma_{1}$ and $\Gamma_{2}, \varepsilon>0$, such that $\rho_{2}\left(\Omega_{1}, \Omega_{2}\right) \leq \varepsilon$ and $2 \kappa_{0} \varepsilon \ll 1$. We assume that there exists a point $A$ of $\Gamma_{1}$ such that $d_{\Gamma_{2}}(A)>\varepsilon$ and prove a contradiction.

Consider the open disk $B(A, \varepsilon)$ of center $A$ and radius $\varepsilon$. This disk does not contain any point of $\Gamma_{2}$ by hypothesis, since otherwise we would have $d_{\Gamma_{2}}(A) \leq \varepsilon$. Moreover, the curve $\Gamma_{1}$ is not included in $B(A, \varepsilon)$ because of the hypothesis $2 \kappa_{0} \varepsilon \ll 1$ and Lemma 21 , therefore there must be a strictly positive even number of points of intersection between $\Gamma_{1}$ and the border of $B(A, \varepsilon)$. If there are more than two, the same reasoning, as in the proof of Proposition 23 below, shows that there is a piece of skeleton of $\Gamma_{1}$ within $B(A, \varepsilon)$ and hence $\Omega_{1} \notin \mathcal{F}_{h_{0}}$.

Let $A_{1}$ and $A_{2}$ be the endpoints of the arc of $\Gamma_{1}$ going through $A$. This arc divides $B(A, \varepsilon)$ in two parts, one of them belongs to $\Omega_{1} \Delta \Omega_{2}$. The idea is that since $2 \varepsilon \kappa_{0} \ll$ 1 , the arc $A_{1} A A_{2}$ is equivalent to a line segment, and each area is approximately equal to $\pi \varepsilon^{2} / 2$, hence $\left\|\chi_{\Omega_{1}}-\chi_{\Omega_{2}}\right\|_{L^{2}} \geq \varepsilon \sqrt{\pi / 2}>\varepsilon$, a contradiction.

In order to prove this, we parameterize $\Gamma_{1}$ between $A_{1}$ and $A_{2}$ by its arc length $s$ and compute an upper-bound on the distance of $A(s)$ to the tangent line to $\partial \Omega_{1}$ at $A$. We choose $A$ as the origin of arc length on $\Gamma_{1}$ and use equation (7):

$$
A(s)=A+s \mathbf{t}(s)+s^{2} \int_{0}^{1}(1-\zeta) \kappa(\zeta s) \mathbf{n}(\zeta s) d \zeta
$$

The distance of $A(s)$ to the line $(A, \mathbf{t}(0))$ is given by

$$
(A(s)-A) \cdot \mathbf{n}(0)=s^{2} \int_{0}^{1}(1-\zeta) \kappa(\zeta s) \mathbf{n}(\zeta s) \cdot \mathbf{n}(0) d \zeta .
$$

We obtain an upper bound on its magnitude by

$$
|(A(s)-A) \cdot \mathbf{n}(0)| \leq \frac{s^{2}}{2} \kappa_{0} .
$$




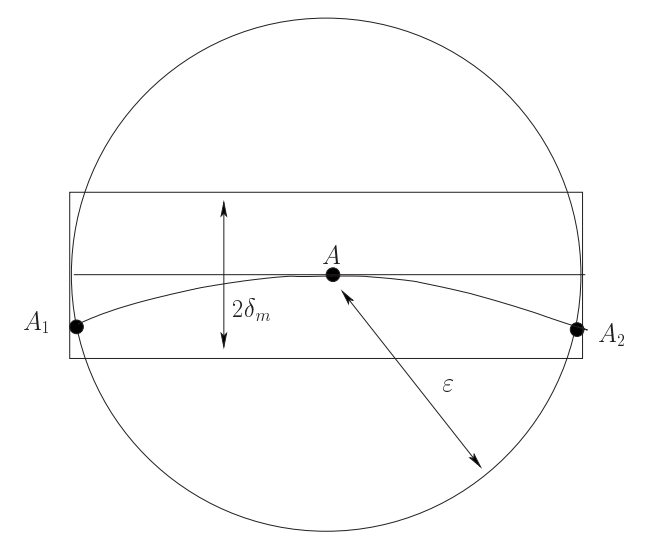

Fig. 5. A lower bound on the area of $\Omega_{1} \Delta \Omega_{2}$ (see text).

The upper bound is maximal for $s=s_{1}\left(A_{1}=A\left(s_{1}\right)\right.$ and $\left.\left|s_{1}\right| \stackrel{\text { def }}{=} \delta_{1}\right)$ or $s=s_{2}$ $\left(A_{2}=A\left(s_{2}\right)\right.$ and $s_{2} \stackrel{\text { def }}{=} \delta_{2}$ ). We obtain upper-bounds from (6); $\delta_{1}$ and $\delta_{2}$ must satisfy

$$
\delta^{2} \frac{\kappa_{0}}{2}-\delta+\varepsilon \geq 0
$$

In order for this to be true we must have

$$
0 \leq \delta \leq \frac{1}{\kappa_{0}}\left(1-\sqrt{1-2 \varepsilon \kappa_{0}}\right) \stackrel{\text { def }}{=} \delta_{m} \quad \text { or } \quad \delta \geq \frac{1}{\kappa_{0}}\left(1+\sqrt{1-2 \varepsilon \kappa_{0}}\right) .
$$

The second alternative is impossible since $B(A, \varepsilon)$ cannot contain an arc whose length is larger than $1 / \kappa_{0}$ (Lemma 21 ). There remains only the first alternative. Returning to (8), we find that $\delta_{m}^{2} \kappa_{0} / 2$ is an upper-bound on the distance of $A(s)$ to the tangent. Referring to Figure 5 we conclude that the area of interest is bounded below by

$$
\frac{\pi \varepsilon^{2}}{2}-\varepsilon \kappa_{0} \delta_{m}^{2}
$$

Since $2 \varepsilon \kappa_{0} \ll 1$, we have $\delta_{m}=\left(1 / \kappa_{0}\right)\left(\varepsilon \kappa_{0}+o\left(\varepsilon \kappa_{0}\right)\right)$ and, therefore,

$$
\varepsilon \kappa_{0} \delta_{m}^{2}=\frac{\varepsilon}{\kappa_{0}}\left(\left(\varepsilon \kappa_{0}\right)^{2}+o\left(\left(\varepsilon \kappa_{0}\right)^{2}\right)\right)=\varepsilon^{2}\left(\varepsilon \kappa_{0}+o\left(\varepsilon \kappa_{0}\right)\right) .
$$

The area of interest is lower, bounded by

$$
\varepsilon^{2}\left(\frac{\pi}{2}-\varepsilon \kappa_{0}+o\left(\varepsilon \kappa_{0}\right)\right)
$$

and, therefore, for $\varepsilon \kappa_{0}$ sufficiently small, its square root is strictly larger than $\varepsilon$.

This completes the proof of Theorem 13. 


\subsection{Minima of a Continuous Function Defined on $\mathcal{S}$}

An interesting and practically important consequence of this analysis of the space $\mathcal{S}$ is the following. We know that $\mathcal{S}$ is included in $\mathcal{D} \mathcal{Z}_{0}$, consider its closure $\mathcal{\mathcal { S }}$ for any one of the three topologies of interest. $\overline{\mathcal{S}}$ is a closed subset of the compact metric space $\mathcal{D} \mathcal{Z}_{0}$ and is therefore compact as well. Given a continuous function $f: \mathcal{S} \rightarrow \mathbb{R}$ we consider its lower semicontinuous (1.s.c.) envelope $f$ defined on $\overline{\mathcal{S}}$ as follows:

$$
\underline{f}(x)=\left\{\begin{array}{l}
f(x) \quad \text { if } \quad x \in \mathcal{S}, \\
\liminf _{y \rightarrow x, y \in \mathcal{S}} f(y) .
\end{array}\right.
$$

The useful result for us is summarized in

Proposition 22. $\quad$ f is l.s.c. in $\overline{\mathcal{S}}$ and therefore has at least a minimum in $\overline{\mathcal{S}}$.

Proof. In a metric space $E$, a real function $f$ is said to be l.s.c. if and only if

$$
f(x) \leq \liminf _{y \rightarrow x} f(y), \quad \text { for all } x \in E .
$$

Therefore $f$ is 1.s.c. by construction. The existence of a minimum of an 1.s.c. function defined on a compact metric space is well-known (see, e.g., [7], [15]) and will be needed later to prove that some of our minimization problems are well-posed.

\section{Deforming Shapes}

The problem of continuously deforming a shape so that it turns into another is central to this paper. The reasons for this will become clearer in the sequel. Let us just mention here that it can be seen as an instance of the warping problem: given two shapes $\Omega_{1}$ and $\Omega_{2}$, how do we deform $\Omega_{1}$ onto $\Omega_{2}$ ? The applications in the field of medical image processing and analysis are immense (see, e.g., [46], [45]). It can also be seen as an instance of the famous (in computer vision) correspondence problem: given two shapes $\Omega_{1}$ and $\Omega_{2}$, how do we find the corresponding point $P_{2}$ in $\Omega_{2}$ of a given point $P_{1}$ in $\Omega_{1}$ ? Note that a solution of the warping problem provides a solution of the correspondence problem if we can track the evolution of any given point during the smooth deformation of the first shape onto the second.

In order to make things more quantitative, we assume that we are given a function $E: \mathcal{C}_{0} \times \mathcal{C}_{0} \rightarrow \mathbb{R}^{+}$, called the energy, which is continuous on $\mathcal{S} \times \mathcal{S}$ for one of the shape topologies of interest. This energy can also be thought of as a measure of the dissimilarity between the two shapes. By smooth, we mean that it is continuous with respect to this topology and that its derivatives are well-defined in a sense we now make more precise.

We first need the notion of a normal deformation flow of a curve $\Gamma$ in $\mathcal{S}$. This is a smooth (i.e., $C^{0}$ ) function $\beta:[0,1] \rightarrow \mathbb{R}$ (when $\Gamma \in \mathcal{S}^{c}$, one further requires 
that $\beta(0)=\beta(1))$. Let $\Gamma:[0,1] \rightarrow \mathbb{R}^{2}$ be a parametrization of $\Gamma, \mathbf{n}(p)$ the unit normal at the point $\Gamma(p)$ of $\Gamma$; the normal deformation flow $\beta$ associates the point $\Gamma(p)+\beta(p) \mathbf{n}(p)$ to $\Gamma(p)$. The resulting shape is noted $\Gamma+\boldsymbol{\beta}$, where $\boldsymbol{\beta}=\beta \mathbf{n}$. There is no guarantee that $\Gamma+\boldsymbol{\beta}$ is still a shape in $\mathcal{S}$ in general but if $\beta$ is $C^{0}$ and $\varepsilon$ is small enough, $\Gamma+\boldsymbol{\beta}$ is in $\mathcal{C}_{0}$. Given two shapes $\Gamma$ and $\Gamma_{0}$, the corresponding energy $E\left(\Gamma, \Gamma_{0}\right)$, and a normal deformation flow $\beta$ of $\Gamma$, the energy $E\left(\Gamma+\varepsilon \boldsymbol{\beta}, \Gamma_{0}\right)$ is now well-defined for $\varepsilon$ sufficiently small. The derivative of $E\left(\Gamma, \Gamma_{0}\right)$ with respect to $\Gamma$ in the direction of the flow $\boldsymbol{\beta}$ is then defined, when it exists, as

$$
\mathcal{G}_{\Gamma}\left(E\left(\Gamma, \Gamma_{0}\right), \boldsymbol{\beta}\right)=\lim _{\varepsilon \rightarrow 0} \frac{E\left(\Gamma+\varepsilon \boldsymbol{\beta}, \Gamma_{0}\right)-E\left(\Gamma, \Gamma_{0}\right)}{\varepsilon} .
$$

This kind of derivative is also known as a Gâteaux semiderivative. In our case the function $\beta \rightarrow \mathcal{G}_{\Gamma}\left(E\left(\Gamma, \Gamma_{0}\right), \boldsymbol{\beta}\right)$ is linear and continuous (it is then called a Gâteaux derivative) and defines a continuous linear form on the vector space of normal deformation flows of $\Gamma$. This is a vector subspace of the Hilbert space $L^{2}(\Gamma)$ with the usual Hilbert product $\left\langle\beta_{1}, \beta_{2}\right\rangle=1 /|\Gamma| \int_{\Gamma} \beta_{1} \beta_{2}=1 /|\Gamma| \int_{\Gamma} \beta_{1}(x) \beta_{2}(x) d \Gamma(x)$, where $|\Gamma|$ is the length of $\Gamma$. Given such an inner product, we can apply Riesz's representation theorem [39] to the Gâteaux derivative $\mathcal{G}_{\Gamma}\left(E\left(\Gamma, \Gamma_{0}\right), \boldsymbol{\beta}\right)$ : There exists a deformation flow, noted $\nabla E\left(\Gamma, \Gamma_{0}\right)$, such that

$$
\mathcal{G}_{\Gamma}\left(E\left(\Gamma, \Gamma_{0}\right), \boldsymbol{\beta}\right)=\left\langle\nabla E\left(\Gamma, \Gamma_{0}\right), \beta\right\rangle .
$$

This flow is called the gradient of $E\left(\Gamma, \Gamma_{0}\right)$.

We now return to the initial problem of smoothly deforming a curve $\Gamma_{1}$ onto a curve $\Gamma_{2}$. We can state it as that of defining a family $\Gamma(t), t \geq 0$, of shapes such that $\Gamma(0)=\Gamma_{1}, \Gamma(T)=\Gamma_{2}$ for some $T>0$, and for each value of $t \geq 0$ the deformation flow of the current shape $\Gamma(t)$ is equal to minus the gradient $\nabla E\left(\Gamma, \Gamma_{2}\right)$ defined previously. This is equivalent to solving the following PDE:

$$
\Gamma_{t}=-\nabla E\left(\Gamma, \Gamma_{2}\right) \mathbf{n}, \quad \Gamma(0)=\Gamma_{1} .
$$

In this paper we do not address the question of the existence of solutions to (10).

Natural candidates for the energy function $E$ are the distances defined in Section 2.2. The problem we are faced with is that none of these distances are Gâteaux differentiable. This is why the next section is devoted to the definition of smooth approximations of some of them.

\section{How to Approximate Shape Distances}

The goal of this section is to provide smooth approximations of some of these distances, i.e., that admit Gâteaux derivatives. We start with some notations. 


\subsection{Averages}

Let $\Gamma$ be a given curve in $\mathcal{C}^{1}$ and consider an integrable function $f: \Gamma \rightarrow \mathbb{R}^{n}$. We denote by $\langle f\rangle_{\Gamma}$ the average of $f$ along the curve $\Gamma$ :

$$
\langle f\rangle_{\Gamma}=\frac{1}{|\Gamma|} \int_{\Gamma} f=\frac{1}{|\Gamma|} \int_{\Gamma} f(x) d \Gamma(x) .
$$

For real positive integrable functions $f$, and for any continuous strictly monotonous (hence one-to-one) function $\varphi$ from $\mathbb{R}^{+}$or $\mathbb{R}^{+*}$ to $\mathbb{R}^{+}$we will also need the $\varphi$ average of $f$ along $\Gamma$ which we define as

$$
\langle f\rangle_{\Gamma}^{\varphi}=\varphi^{-1}\left(\frac{1}{|\Gamma|} \int_{\Gamma} \varphi \circ f\right)=\varphi^{-1}\left(\frac{1}{|\Gamma|} \int_{\Gamma} \varphi(f(x)) d \Gamma(x)\right) .
$$

Note that $\varphi^{-1}$ is also strictly monotonous and continous from $\mathbb{R}^{+}$to $\mathbb{R}^{+}$or $\mathbb{R}^{+*}$. Also note that the unit of the $\varphi$-average of $f$ is the same as that of $f$, thanks to the normalization by $|\Gamma|$.

The discrete version of the $\varphi$-average is also useful: let $a_{i}, i=1, \ldots, n$, be $n$ positive numbers, we note

$$
\left\langle a_{1}, \ldots, a_{n}\right\rangle^{\varphi}=\varphi^{-1}\left(\frac{1}{n} \sum_{i=1}^{n} \varphi\left(a_{i}\right)\right)
$$

their $\varphi$-average.

\subsection{Approximations of the Hausdorff Distance}

We now build a series of smooth approximations of the Hausdorff distance $\rho_{H}$ $\left(\Gamma, \Gamma^{\prime}\right)$ of two shapes $\Gamma$ and $\Gamma^{\prime}$. According to (4) we have to consider the functions $d_{\Gamma^{\prime}}: \Gamma \rightarrow \mathbb{R}^{+}$and $d_{\Gamma}: \Gamma^{\prime} \rightarrow \mathbb{R}^{+}$. Let us focus on the second one. Since $d_{\Gamma}$ is Lipschitz continuous on the bounded hold-all set $D$ it is certainly integrable on the compact set $\Gamma^{\prime}$ and we have [39, Chap. 3, Prob. 4]

$$
\lim _{\beta \rightarrow+\infty}\left(\frac{1}{\left|\Gamma^{\prime}\right|} \int_{\Gamma^{\prime}} d_{\Gamma}^{\beta}\left(x^{\prime}\right) d \Gamma^{\prime}\left(x^{\prime}\right)\right)^{1 / \beta}=\sup _{x^{\prime} \in \Gamma^{\prime}} d_{\Gamma}\left(x^{\prime}\right) .
$$

Moreover, the function $\mathbb{R}^{+} \rightarrow \mathbb{R}^{+}$defined by $\beta \rightarrow\left(1 /\left|\Gamma^{\prime}\right| \int_{\Gamma^{\prime}} d_{\Gamma}^{\beta}\left(x^{\prime}\right) d \Gamma^{\prime}\left(x^{\prime}\right)\right)^{1 / \beta}$ is monotonously increasing [39, Chap. 3, Prob. 5].

Similar properties hold for $d_{\Gamma^{\prime}}$.

If we note by $p_{\beta}$ the function $\mathbb{R}^{+} \rightarrow \mathbb{R}^{+}$defined by $p_{\beta}(x)=x^{\beta}$ we can rewrite (14),

$$
\lim _{\beta \rightarrow+\infty}\left\langle d_{\Gamma}\right\rangle_{\Gamma^{\prime}}^{p_{\beta}}=\sup _{x^{\prime} \in \Gamma^{\prime}} d_{\Gamma}\left(x^{\prime}\right) .
$$


$\left\langle d_{\Gamma}\right\rangle_{\Gamma^{\prime}}^{p_{\beta}}$ is therefore a monotonically increasing approximation of $\sup _{x^{\prime} \in \Gamma^{\prime}} d_{\Gamma}\left(x^{\prime}\right)$. We go one step further and approximate $d_{\Gamma^{\prime}}(x)$.

Consider a continuous strictly monotonously decreasing function $\varphi: \mathbb{R}^{+} \rightarrow$ $\mathbb{R}^{+*}$. Because $\varphi$ is strictly monotonously decreasing

$$
\sup _{x^{\prime} \in \Gamma^{\prime}} \varphi\left(d\left(x, x^{\prime}\right)\right)=\varphi\left(\inf _{x^{\prime} \in \Gamma^{\prime}} d\left(x, x^{\prime}\right)\right)=\varphi\left(d_{\Gamma^{\prime}}(x)\right),
$$

and, moreover,

$$
\lim _{\alpha \rightarrow+\infty}\left(\frac{1}{\left|\Gamma^{\prime}\right|} \int_{\Gamma^{\prime}} \varphi^{\alpha}\left(d\left(x, x^{\prime}\right)\right) d \Gamma^{\prime}\left(x^{\prime}\right)\right)^{1 / \alpha}=\sup _{x^{\prime} \in \Gamma^{\prime}} \varphi\left(d\left(x, x^{\prime}\right)\right) .
$$

Because $\varphi$ is continuous and strictly monotonously decreasing, it is one-to-one and $\varphi^{-1}$ is strictly monotonously decreasing and continuous. Therefore

$$
d_{\Gamma^{\prime}}(x)=\lim _{\alpha \rightarrow+\infty} \varphi^{-1}\left(\left(\frac{1}{\left|\Gamma^{\prime}\right|} \int_{\Gamma^{\prime}} \varphi^{\alpha}\left(d\left(x, x^{\prime}\right)\right) d \Gamma^{\prime}\left(x^{\prime}\right)\right)^{1 / \alpha}\right) .
$$

We can simplify this equation by introducing the function $\varphi_{\alpha}=p_{\alpha} \circ \varphi$ :

$$
d_{\Gamma^{\prime}}(x)=\lim _{\alpha \rightarrow+\infty}\langle d(x, \cdot)\rangle_{\Gamma^{\prime}}^{\varphi_{\alpha}} .
$$

Any $\alpha>0$ provides us with an approximation, noted $\tilde{d}_{\Gamma^{\prime}}$, of $d_{\Gamma^{\prime}}$ :

$$
\tilde{d}_{\Gamma^{\prime}}(x)=\langle d(x, \cdot)\rangle_{\Gamma^{\prime}}^{\varphi_{\alpha}}
$$

We have a similar expression for $\tilde{d}_{\Gamma}$.

Note that because $\left(1 /\left|\Gamma^{\prime}\right| \int_{\Gamma^{\prime}} \varphi^{\alpha}\left(d\left(x, x^{\prime}\right)\right) d \Gamma^{\prime}\left(x^{\prime}\right)\right)^{1 / \alpha}$ increases with $\alpha$ toward its limit $\sup _{x^{\prime}} \varphi\left(d\left(x, x^{\prime}\right)\right)=\varphi\left(d_{\Gamma^{\prime}}(x)\right), \varphi^{-1}\left(\left(1 /\left|\Gamma^{\prime}\right| \int_{\Gamma^{\prime}} \varphi^{\alpha}\left(d\left(x, x^{\prime}\right)\right) d \Gamma^{\prime}\left(x^{\prime}\right)\right)^{1 / \alpha}\right)$ decreases with $\alpha$ toward its limit $d_{\Gamma^{\prime}}(x)$. Examples of functions $\varphi$ are

$$
\begin{aligned}
& \varphi_{1}(z)=\frac{1}{z+\varepsilon}, \quad \varepsilon>0, z \geq 0, \\
& \varphi_{2}(z)=\mu \exp (-\lambda z), \quad \mu, \lambda>0, z \geq 0, \\
& \varphi_{3}(z)=\frac{1}{\sqrt{2 \pi \sigma^{2}}} \exp \left(-\frac{z^{2}}{2 \sigma^{2}}\right), \quad \sigma>0, z \geq 0 .
\end{aligned}
$$

Putting all this together we have the following result:

$$
\begin{aligned}
& \sup _{x \in \Gamma} d_{\Gamma^{\prime}}(x)=\lim _{\alpha, \beta \rightarrow+\infty}\left\langle\langle d(\cdot, \cdot)\rangle_{\Gamma^{\prime}}^{\varphi_{\alpha}}\right\rangle_{\Gamma}^{p_{\beta}}, \\
& \sup _{x \in \Gamma^{\prime}} d_{\Gamma}(x)=\lim _{\alpha, \beta \rightarrow+\infty}\left\langle\langle d(\cdot, \cdot)\rangle_{\Gamma}^{\varphi_{\alpha}}\right\rangle_{\Gamma^{\prime}}^{p_{\beta}} .
\end{aligned}
$$

Any positive values of $\alpha$ and $\beta$ yield approximations of $\sup _{x \in \Gamma} d_{\Gamma^{\prime}}(x)$ and $\sup _{x \in \Gamma^{\prime}}$ $d_{\Gamma}(x)$. 
The last point to address is the max that appears in the definition of the Hausdorff distance. We use (13), choose $\varphi=p_{\gamma}$, and note that, for $a_{1}$ and $a_{2}$ positive,

$$
\lim _{\gamma \rightarrow+\infty}\left\langle a_{1}, a_{2}\right\rangle^{p_{\gamma}}=\max \left(a_{1}, a_{2}\right)
$$

This yields the following expression for the Hausdorff distance between two shapes $\Gamma$ and $\Gamma^{\prime}$ :

$$
\rho_{H}\left(\Gamma, \Gamma^{\prime}\right)=\lim _{\alpha, \beta, \gamma \rightarrow+\infty}\left\langle\left\langle\langle d(\cdot, \cdot)\rangle_{\Gamma^{\prime}}^{\varphi_{\alpha}}\right\rangle_{\Gamma}^{p_{\beta}},\left\langle\langle d(\cdot, \cdot)\rangle_{\Gamma}^{\varphi_{\alpha}}\right\rangle_{\Gamma^{\prime}}^{p_{\beta}}\right\rangle^{p_{\gamma}}
$$

This equation is symmetric and yields approximations $\tilde{\rho}_{H}$ of the Hausdorff distance for all positive values of $\alpha, \beta$, and $\gamma$ :

$$
\tilde{\rho}_{H}\left(\Gamma, \Gamma^{\prime}\right)=\left\langle\left\langle\langle d(\cdot, \cdot)\rangle_{\Gamma^{\prime}}^{\varphi_{\alpha}}\right\rangle_{\Gamma}^{p_{\beta}},\left\langle\langle d(\cdot, \cdot)\rangle_{\Gamma}^{\varphi_{\alpha}}\right\rangle_{\Gamma^{\prime}}^{p_{\beta}}\right\rangle^{p_{\gamma}}
$$

This approximation is "nice" in several ways, the first one being the obvious one, stated in the following:

Proposition 23. For each triplet $(\alpha, \beta, \gamma)$ in $\left(\mathbb{R}^{+*}\right)^{3}$ the function $\tilde{\rho}_{H}: \mathcal{S} \times \mathcal{S} \rightarrow$ $\mathbb{R}^{+}$, defined by equation (18), is continuous for the Hausdorff topology.

We first recall the following properties of the squared distance function $\eta_{\partial \Omega}$ of the boundary of an element $\Omega$ of $\mathcal{S}$ (see [2]):

Proposition 24. $\eta_{\partial \Omega}$ is smooth, i.e., $C^{2}$, in $U_{h_{0}}(\partial \Omega)$ and for all $x \in \partial \Omega$, the Hessian matrix $\nabla^{2} \eta_{\partial \Omega}(x)$ is the (matrix of) orthogonal projection onto the normal to $\partial \Omega$ at $x$.

We now prove Proposition 23.

Proof. For each shape $\Omega$ of $\mathcal{S}$, we consider the square of the distance function of $\partial \Omega$, denoted $\eta_{\partial \Omega}$. We next prove that the length is continuous for the Hausdorff topology on $\mathcal{S}$. Consider two shapes $\Omega_{1}$ and $\Omega_{2}$ of $\mathcal{S}$, their boundaries $\Gamma_{1}$ and $\Gamma_{2}$, and assume that $\rho_{H}\left(\Gamma_{1}, \Gamma_{2}\right)<\varepsilon$. Let $p \in[0,1] \rightarrow \Gamma_{1}(p)$ be a $C^{2}$ parametrization of $\Gamma_{1}$, we prove that the mapping

$$
p \in[0,1] \rightarrow \Gamma_{2}(p)=\Gamma_{1}(p)-\frac{1}{2} \nabla \eta_{\Gamma_{2}}\left(\Gamma_{1}(p)\right)
$$

is a one-to-one parametrization of $\Gamma_{2}$. If we choose $\varepsilon<h_{0}, \nabla \eta_{\Gamma_{2}}\left(\Gamma_{1}(p)\right)$ is well-defined and continuous for all $p$ 's (Proposition 24), hence $p \rightarrow \Gamma_{1}(p)-$ $\frac{1}{2} \nabla \eta_{\Gamma_{2}}\left(\Gamma_{1}(p)\right)$ is continuous.

It is injective: Assume that there exist $p_{1}$ and $p_{2}$ in $[0,1], p_{1} \neq p_{2}$, such that $\Gamma_{2}\left(p_{1}\right)=\Gamma_{2}\left(p_{2}\right)$, see Figure 6 (if $\Gamma_{1}$ and $\Gamma_{2}$ are closed, $p_{1}, p_{2} \notin\{0,1\}$ ). Since the curvature of $\Gamma_{1}$ and $\Gamma_{2}$ is bounded by $1 / h_{0}$, we choose $\varepsilon \ll h_{0}$. The two points $\Gamma_{1}\left(p_{1}\right)$ and $\Gamma_{1}\left(p_{2}\right)$ are in the disk of center $\Gamma_{2}\left(p_{1}\right)$ and radius $\varepsilon$ since their distances 


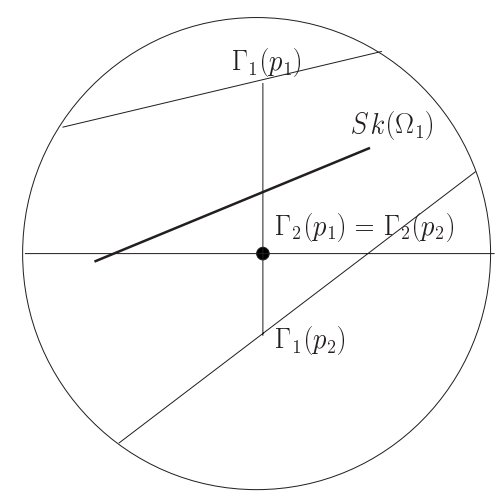

Fig. 6. The mapping is injective.

to $\Gamma_{2}$ are by construction equal to $d\left(\Gamma_{1}\left(p_{1}\right), \Gamma_{2}\left(p_{1}\right)\right)$ and $d\left(\Gamma_{1}\left(p_{2}\right), \Gamma_{2}\left(p_{2}\right)\right)$, respectively, and are less than $\varepsilon$. Because of our choice of $\varepsilon$, the curvatures of the two curves within the disk are negligible and we can consider they are straight lines, as shown in Figure 6. Therefore there must be a piece of the skeleton of $\Omega_{1}$ within the disk and this contradicts the hypothesis that $\Omega_{1} \in \mathcal{F}_{h_{0}}$.

It is surjective: We proceed by contradiction. Let us assume it is not surjective. Since the mapping (19) is continuous its image is connected and compact. Its complement $\hat{\Gamma}_{2}$ (assumed here to be nonempty) is thus an open interval of $\Gamma_{2}$ (possibly two, if $\Gamma_{2}$ is an open curve). Let $\Gamma_{2}^{0}$ be one of the endpoints of this interval. There exists a value $p_{0}$ of $p$ such that

$$
\Gamma_{2}^{0}=\Gamma_{1}\left(p_{0}\right)-\frac{1}{2} \nabla \eta_{\Gamma_{2}}\left(\Gamma_{1}\left(p_{0}\right)\right)
$$

Two cases can occur. Either $\Gamma_{2}^{0}=\Gamma_{1}\left(p_{0}\right)$ and this implies that $\Gamma_{2}$ is not simple (see Figure 7(a)), or $\Gamma_{2}^{0} \neq \Gamma_{1}\left(p_{0}\right)$ and this implies that $\Gamma_{1}\left(p_{0}\right)$ is on the skeleton of $\Gamma_{2}$, a contradiction if $\varepsilon$ is small with respect to $h_{0}$ (see Figure 7(b)).

Using this parametrization, we now prove that the length is continuous for the Hausdorff metric. Given a shape $\Omega$ and a sequence $\left\{\Omega_{n}\right\}$ of shapes of $\mathcal{S}$ such that the boundaries $\Gamma_{n}$ are converging to the boundary $\Gamma$ of $\Omega$ for the Hausdorff topology, we show that $\lim _{n \rightarrow \infty}|| \Gamma_{n}|-| \Gamma||=0$. If $n$ is large enough, we use the first part of the proof to parametrize $\Gamma_{n}$ :

$$
\Gamma_{n}(p)=\Gamma(p)-\frac{1}{2} \nabla \eta_{\Gamma_{n}}(\Gamma(p)),
$$

and proceed from there:

$$
\begin{aligned}
|| \partial \Omega_{n}|-| \partial \Omega|| & =\left|\int_{0}^{1}\right| \Gamma_{n}^{\prime}(p)\left|d p-\int_{0}^{1}\right| \Gamma^{\prime}(p)|d p| \\
& \leq \int_{0}^{1}|| \Gamma_{n}^{\prime}(p)|-| \Gamma^{\prime}(p)|| d p \leq \int_{0}^{1}\left|\Gamma_{n}^{\prime}(p)-\Gamma^{\prime}(p)\right| d p .
\end{aligned}
$$




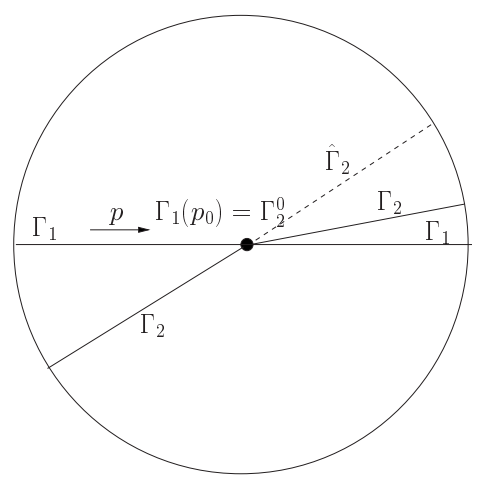

(a)

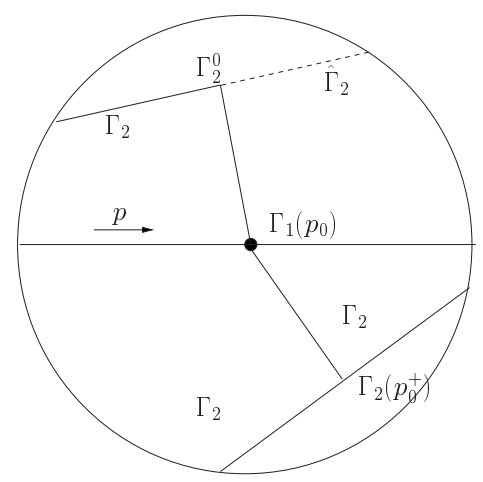

(b)

Fig. 7. The mapping is surjective: The dotted line represents a piece of $\hat{\Gamma}_{2}$, see text.

We take the derivative of (20) with respect to $p$ :

$$
\Gamma_{n}^{\prime}(p)=\Gamma^{\prime}(p)-\frac{1}{2} \nabla^{2} \eta_{\Gamma_{n}}(\Gamma(p)) \Gamma^{\prime}(p),
$$

where $\nabla^{2} \eta_{\Gamma_{n}}$ is the second-order derivative of $\eta_{\Gamma_{n}}$.

We are only interested in comparing the lengths of $\Gamma$ and $\Gamma_{n}$ where they differ. We can therefore exclude from the integral $\int\left|\Gamma_{n}^{\prime}(p)-\Gamma^{\prime}(p)\right| d p$ the values of $p$ for which $\Gamma_{n}(p)=\Gamma(p)$ and assume that $\Gamma_{n}(p) \neq \Gamma(p)$. At these points, the first- and second-order derivatives of the distance function $d_{\Gamma_{n}}$ are well-defined and (because $\Gamma_{n} \in \mathcal{S}$ and $\varepsilon \ll h_{0}$ ) there exists $M>0$, independent of $n$, such that

$$
\left|\nabla^{2} d_{\Gamma_{n}}(x)\right| \leq M, \quad \forall x \notin \Gamma_{n} .
$$

Using the chain rule we obtain

$$
\frac{1}{2} \nabla \eta_{\Gamma_{n}}=d_{\Gamma_{n}} \nabla d_{\Gamma_{n}}, \quad \frac{1}{2} \nabla^{2} \eta_{\Gamma_{n}}=d_{\Gamma_{n}} \nabla^{2} d_{\Gamma_{n}}+\nabla d_{\Gamma_{n}}\left(\nabla d_{\Gamma_{n}}\right)^{T},
$$

and, therefore,

$$
\begin{aligned}
\left|\Gamma_{n}^{\prime}(p)-\Gamma^{\prime}(p)\right| \leq \mid & \left|\nabla d_{\Gamma_{n}}(\Gamma(p)) \cdot \Gamma^{\prime}(p)\right|\left|\nabla d_{\Gamma_{n}}(\Gamma(p))\right| \\
& +d_{\Gamma_{n}}(\Gamma(p))\left\|\nabla^{2} d_{\Gamma_{n}}(\Gamma(p)) \Gamma^{\prime}(p)\right\| \\
\leq \mid & \left|\nabla d_{\Gamma_{n}}(\Gamma(p)) \cdot \Gamma^{\prime}(p)\right|+M d_{\Gamma_{n}}(\Gamma(p))\left|\Gamma^{\prime}(p)\right| .
\end{aligned}
$$

Consider the term $\nabla d_{\Gamma_{n}}(\Gamma(p)) \cdot \Gamma^{\prime}(p)$. We write the following first-order Taylor expansion without remainder:

$$
\begin{aligned}
0= & d_{\Gamma_{n}}\left(\Gamma_{n}(p)\right)=d_{\Gamma_{n}}(\Gamma(p)) \\
& +\left(\int_{0}^{1}(1-\zeta) \nabla d_{\Gamma_{n}}\left(\Gamma(p)+\zeta\left(\Gamma_{n}(p)-\Gamma(p)\right)\right) d \zeta\right) \cdot\left(\Gamma_{n}(p)-\Gamma(p)\right) .
\end{aligned}
$$


We take the derivative with respect to $p$ :

$$
\begin{aligned}
& \nabla d_{\Gamma_{n}}(\Gamma(p)) \cdot \Gamma^{\prime}(p) \\
& \quad+\left(\int_{0}^{1}(1-\zeta) \nabla d_{\Gamma_{n}}\left(\Gamma(p)+\zeta\left(\Gamma_{n}(p)-\Gamma(p)\right)\right) d \zeta\right) \cdot\left(\Gamma_{n}^{\prime}(p)-\Gamma^{\prime}(p)\right) \\
& \quad+\left(\int_{0}^{1}(1-\zeta) \nabla^{2} d_{\Gamma_{n}}\left(\Gamma(p)+\zeta\left(\Gamma_{n}(p)-\Gamma(p)\right)\right)\right. \\
& \left.\quad \times\left(\Gamma^{\prime}(p)+\zeta\left(\Gamma_{n}^{\prime}(p)-\Gamma^{\prime}(p)\right)\right) d \zeta\right) \cdot\left(\Gamma_{n}(p)-\Gamma(p)\right),
\end{aligned}
$$

and obtain the upper bound

$$
\left|\nabla d_{\Gamma_{n}}(\Gamma(p)) \cdot \Gamma^{\prime}(p)\right| \leq \frac{1}{2}\left|\Gamma_{n}^{\prime}(p)-\Gamma^{\prime}(p)\right|+\operatorname{Ad}\left(\Gamma_{n}(p), \Gamma(p)\right) .
$$

We use this in (21) to obtain

$$
\frac{1}{2}\left|\Gamma_{n}^{\prime}(p)-\Gamma^{\prime}(p)\right| \leq A d\left(\Gamma_{n}(p), \Gamma(p)\right)+M d_{\Gamma_{n}}(\Gamma(p))\left|\Gamma^{\prime}(p)\right|,
$$

from which follows that

$$
|| \Gamma_{n}|-| \Gamma|| \leq 2(A+M|\Gamma|) \varepsilon .
$$

We next prove that for all Lipschitz continuous functions $f$ on $D$, the integral $\int_{\Gamma} f(x) d \Gamma(x)$ is continuous for the Hausdorff topology. Consider a shape $\Omega$ and a sequence $\left\{\Omega_{n}\right\}$ of shapes of $\mathcal{S}$ whose boundaries $\Gamma_{n}$ are converging to the boundary $\Gamma$ of $\Omega$ for the Hausdorff topology; we show that $\lim _{n \rightarrow \infty} \mid \int_{\Gamma_{n}} f(x) d \Gamma_{n}(x)-$ $\int_{\Gamma} f(y) d \Gamma(y) \mid=0$. We use once more the parametrization (20) and write

$$
\begin{aligned}
&\left|\int_{\Gamma_{n}} f(x) d \Gamma_{n}(x)-\int_{\Gamma} f(y) d \Gamma(y)\right| \\
&=\left|\int_{0}^{1}\left(f\left(\Gamma_{n}(p)\right)\left|\Gamma_{n}^{\prime}(p)\right|-f(\Gamma(p))\left|\Gamma^{\prime}(p)\right|\right) d p\right| \\
& \leq \int_{0}^{1}\left|f\left(\Gamma_{n}(p)\right)\right| \Gamma_{n}^{\prime}(p)|-f(\Gamma(p))| \Gamma^{\prime}(p)|| d p \\
& \leq \int_{0}^{1}\left|f\left(\Gamma_{n}(p)\right)\right||| \Gamma_{n}^{\prime}(p)|-| \Gamma^{\prime}(p)|| d p \\
&+\int_{0}^{1}\left|f\left(\Gamma_{n}(p)\right)-f(\Gamma(p))\right|\left|\Gamma^{\prime}(p)\right| d p
\end{aligned}
$$

where $f$ is continuous on the compact set $\bar{D}$ and is therefore upper-bounded, $|f(x)| \leq K$, for all $x \in \bar{D}$. It is also Lipschitz continuous, hence $\mid f\left(\Gamma_{n}(p)\right)-$ $f(\Gamma(p)) \mid \leq L d\left(\Gamma_{n}(p), \Gamma(p)\right) \leq L \varepsilon$. We combine this with (22) and obtain

$$
\left|\int_{\Gamma_{n}} f(x) d \Gamma_{n}(x)-\int_{\Gamma} f(y) d \Gamma(y)\right| \leq \varepsilon((L+2 K M)|\Gamma|+2 K A) .
$$


We have used the Lipschitz hypothesis in the proof. It easy to verify that this hypothesis is satisfied since we are integrating along curves functions of the type $\varphi \circ d(\cdot, x)$. The functions $\varphi$ are defined and at least $C^{1}$, hence Lipschitz continuous on $[0, \operatorname{diam}(D)]$, where $\operatorname{diam}(D)$ is the diameter of $D$. Hence $\mid \varphi \circ d\left(x_{1}, x\right)-\varphi \circ$ $d\left(x_{2}, x\right)\left|\leq L_{\varphi}\right| d\left(x_{1}, x\right)-d\left(x_{2}, x\right) \mid \leq L_{\varphi} d\left(x_{1}, x_{2}\right)$.

\subsection{Computing the Gradient of the Approximation to the Hausdorff Distance}

We now proceed with showing that the approximation $\tilde{\rho}_{H}\left(\Gamma, \Gamma_{0}\right)$ of the Hausdorff distance $\rho_{H}\left(\Gamma, \Gamma_{0}\right)$ is differentiable with respect to $\Gamma$ and compute its gradient $\nabla \tilde{\rho}_{H}\left(\Gamma, \Gamma_{0}\right)$, in the sense of Section 4 . To simplify notations we rewrite (18) as

$$
\tilde{\rho}_{H}\left(\Gamma, \Gamma_{0}\right)=\left\langle\left\langle\langle d(\cdot, \cdot)\rangle_{\Gamma_{0}}^{\varphi}\right\rangle_{\Gamma}^{\psi},\left\langle\langle d(\cdot, \cdot)\rangle_{\Gamma}^{\varphi}\right\rangle_{\Gamma_{0}}^{\psi}\right\rangle^{\theta},
$$

and state the result, the reader interested in the proof being referred to Appendix A.

Proposition 25. The gradient of $\tilde{\rho}_{H}\left(\Gamma, \Gamma_{0}\right)$ at any point $y$ of $\Gamma$ is given by

$$
\nabla \tilde{\rho}_{H}\left(\Gamma, \Gamma_{0}\right)(y)=\frac{1}{\theta^{\prime}\left(\tilde{\rho}_{H}\left(\Gamma, \Gamma_{0}\right)\right)}(\alpha(y) \kappa(y)+\beta(y)),
$$

where $\kappa(y)$ is the curvature of $\Gamma$ at $y$, the functions $\alpha(y)$ and $\beta(y)$ are given by

$$
\begin{aligned}
\alpha(y)= & v \int_{\Gamma_{0}} \frac{\psi^{\prime}}{\varphi^{\prime}}\left(\langle d(x, \cdot)\rangle_{\Gamma}^{\varphi}\right)\left[\varphi \circ\langle d(x, \cdot)\rangle_{\Gamma}^{\varphi}-\varphi \circ d(x, y)\right] d \Gamma_{0}(x) \\
& +\left|\Gamma_{0}\right| \eta\left[\psi\left(\left\langle\langle d(\cdot, \cdot)\rangle_{\Gamma_{0}}^{\varphi}\right\rangle_{\Gamma}^{\psi}\right)-\psi\left(\langle d(\cdot, y)\rangle_{\Gamma_{0}}^{\varphi}\right)\right], \\
\beta(y)= & \int_{\Gamma_{0}} \varphi^{\prime} \circ d(x, y)\left[\nu \frac{\psi^{\prime}}{\varphi^{\prime}}\left(\langle d(x, \cdot)\rangle_{\Gamma}^{\varphi}\right)+\eta \frac{\psi^{\prime}}{\varphi^{\prime}}\left(\langle d(\cdot, y)\rangle_{\Gamma_{0}}^{\varphi}\right)\right] \\
& \frac{y-x}{d(x, y)} \cdot n(y) d \Gamma_{0}(x)
\end{aligned}
$$

where

$$
v=\frac{1}{|\Gamma|\left|\Gamma_{0}\right|} \frac{\theta^{\prime}}{\psi^{\prime}}\left(\left\langle\langle d(\cdot, \cdot)\rangle_{\Gamma}^{\varphi}\right\rangle_{\Gamma_{0}}^{\psi}\right) \quad \text { and } \quad \eta=\frac{1}{|\Gamma|\left|\Gamma_{0}\right|} \frac{\theta^{\prime}}{\psi^{\prime}}\left(\left\langle\langle d(\cdot, \cdot)\rangle_{\Gamma_{0}}^{\varphi}\right\rangle_{\Gamma}^{\psi}\right) \text {. }
$$

Note that the function $\beta(y)$ is well-defined even if $y$ belongs to $\Gamma_{0}$ since the term $(y-x) / d(x, y)$ is of unit norm.

The first two terms of the gradient show explicitly that minimizing the energy implies homogenizing the distance to $\Gamma_{0}$ along the curve $\Gamma$, that is to say, the algorithm will take care in priority of the points of $\Gamma$ which are the furthest from $\Gamma_{0}$.

We should also note that this result holds independently of the fact that the curves are open or closed (see Definition 9 and Appendix A). 


\subsection{Other Alternatives Related to the Hausdorff Distance}

There exist several alternatives to the method presented in the previous sections if we use $\rho$ (equation (3)) rather than $\rho_{H}$ (equation (4)) to define the Hausdorff distance. A first alternative is to use the following approximation:

$$
\tilde{\rho}\left(\Gamma, \Gamma^{\prime}\right)=\left\langle\left|d_{\Gamma}-d_{\Gamma^{\prime}}\right|\right\rangle_{D}^{p_{\alpha}},
$$

where the bracket $\langle f(\cdot)\rangle_{D}^{\varphi}$ is defined in the obvious way for any integrable function $f: D \rightarrow \mathbb{R}^{+}$,

$$
\langle f\rangle_{D}^{\varphi}=\varphi^{-1}\left(\frac{1}{m(D)} \int_{D} \varphi(f(x)) d x\right),
$$

and which can be minimized, as in Section 5.6, with respect to $d_{\Gamma}$. A second alternative is to approximate $\rho$ using

$$
\tilde{\rho}\left(\Gamma, \Gamma^{\prime}\right)=\left\langle\left|\langle d(\cdot, \cdot)\rangle_{\Gamma^{\prime}}^{\varphi_{\beta}}-\langle d(\cdot, \cdot)\rangle_{\Gamma}^{\varphi_{\beta}}\right|\right\rangle_{D}^{p_{\alpha}},
$$

and to compute its derivative with respect to $\Gamma$, as we did in the previous section for $\tilde{\rho}_{H}$.

\subsection{Approximations to the $W^{1,2}$ Norm and Computation of Their Gradient}

The previous results can be used to construct approximations $\tilde{\rho}_{D}$ to the distance $\rho_{D}$ defined in Section 2.2.3:

$$
\tilde{\rho}_{D}\left(\Gamma_{1}, \Gamma_{2}\right)=\left\|\tilde{d}_{\Gamma_{1}}-\tilde{d}_{\Gamma_{2}}\right\|_{W^{1,2}(D)},
$$

where $\tilde{d}_{\Gamma_{i}}, i=1,2$, is obtained from (16).

This approximation is also "nice" in the usual way and we have

Proposition 26. For each $\alpha$ in $\mathbb{R}^{+*}$ the function $\tilde{\rho}_{D}: \mathcal{S} \times \mathcal{S} \rightarrow \mathbb{R}^{+}$is continuous for the $W^{1,2}$ topology.

Its proof is left to the reader.

The gradient $\nabla \tilde{\rho}_{D}\left(\Gamma, \Gamma_{0}\right)$ of our approximation $\tilde{\rho}_{D}\left(\Gamma, \Gamma_{0}\right)$ of the distance $\rho_{D}\left(\Gamma, \Gamma_{0}\right)$, given by (28) in the sense of Section 4 , can be computed. The interested reader is referred to Appendix B. We simply state the result in

Proposition 27. The gradient of $\tilde{\rho}_{D}\left(\Gamma, \Gamma_{0}\right)$ at any point $y$ of $\Gamma$ is given by

$$
\begin{aligned}
\nabla \tilde{\rho}_{D}\left(\Gamma, \Gamma_{0}\right)(y) & =\int_{D}\left[B(x, y)\left(C_{1}(x)-\frac{\varphi^{\prime \prime}}{\varphi^{\prime}}\left(\tilde{d}_{\Gamma}(x)\right)\left(\mathbf{C}_{2}(x) \cdot \nabla \tilde{d}_{\Gamma}(x)\right)\right)\right. \\
& \left.+\mathbf{C}_{2}(x) \cdot \nabla B(x, y)\right] d x
\end{aligned}
$$


where

$$
B(x, y)=\kappa(y)\left(\langle\varphi \circ d(x, \cdot)\rangle_{\Gamma}-\varphi \circ d(x, y)\right)+\varphi^{\prime}(d(x, y)) \frac{y-x}{d(x, y)} \cdot \mathbf{n}(y),
$$

where $\kappa(y)$ is the curvature of $\Gamma$ at $y$,

$$
C_{1}(x)=\frac{1}{|\Gamma| \varphi^{\prime}\left(\tilde{d}_{\Gamma}(x)\right)}\left\|\tilde{d}_{\Gamma}-\tilde{d}_{\Gamma_{0}}\right\|_{L^{2}(D)}^{-1}\left(\tilde{d}_{\Gamma}(x)-\tilde{d}_{\Gamma_{0}}(x)\right),
$$

and

$$
\mathbf{C}_{2}(x)=\frac{1}{|\Gamma| \varphi^{\prime}\left(\tilde{d}_{\Gamma}(x)\right)}\left\|\nabla\left(\tilde{d}_{\Gamma}-\tilde{d}_{\Gamma_{0}}\right)\right\|_{\mathbf{L}^{2}(D)}^{-1} \nabla\left(\tilde{d}_{\Gamma}-\tilde{d}_{\Gamma_{0}}\right)(x) .
$$

\subsection{Direct Minimization of the $W^{1,2}$ Norm}

An alternative to the method presented in the previous section is to evolve not the curve $\Gamma$ but its distance function $d_{\Gamma}$. Minimizing $\rho_{D}\left(\Gamma, \Gamma_{0}\right)$ with respect to $d_{\Gamma}$ implies computing the corresponding Euler-Lagrange equation $E L$. The reader will verify that the result is

$$
E L=\frac{d_{\Gamma}-d_{\Gamma_{0}}}{\left\|d_{\Gamma}-d_{\Gamma_{0}}\right\|_{L^{2}(D)}}-\operatorname{div}\left(\frac{\nabla\left(d_{\Gamma}-d_{\Gamma_{0}}\right)}{\left.\left\|\nabla\left(d_{\Gamma}-d_{\Gamma_{0}}\right)\right\|_{\mathbf{L}^{2}(D)}\right)}\right) .
$$

To simplify notations we now use $d$ instead of $d_{\Gamma}$. The problem of warping $\Gamma_{1}$ onto $\Gamma_{0}$ is then transformed into the problem of solving the following PDE:

$$
\begin{aligned}
d_{t} & =-E L, \\
d(0, \cdot) & =d_{\Gamma_{1}}(\cdot) .
\end{aligned}
$$

The problem, that this PDE does not preserve the fact that $d$ is a distance function, is alleviated by "reprojecting" at each iteration the current function $d$ onto the set of distance functions by running a few iterations of the "standard" restoration PDE [44]

$$
\begin{aligned}
d_{t} & =(1-|\nabla d|) \operatorname{sign}\left(d_{0}\right), \\
d(0, \cdot) & =d_{0} .
\end{aligned}
$$

\section{Application to Curve Evolutions: Hausdorff Warping}

In this section we show a number of examples of solving equation (10) with the gradient given by equation (24). Our hope is that, starting from $\Gamma_{1}$, we will follow the gradient (24) and smoothly converge to the curve $\Gamma_{2}$ where the minimum of $\tilde{\rho}_{H}$ is attained. Let us examine more closely these assumptions. First, it is clear from 
expression (18) of $\tilde{\rho}_{H}$ that in general $\tilde{\rho}_{H}(\Gamma, \Gamma) \neq 0$, which implies in particular that $\tilde{\rho}_{H}$, unlike $\rho_{H}$, is not a distance. But worse things can happen: there may exist a shape $\Gamma^{\prime}$ such that $\tilde{\rho}_{H}\left(\Gamma, \Gamma^{\prime}\right)$ is strictly less than $\tilde{\rho}_{H}(\Gamma, \Gamma)$ or there may not exist any minima for the function $\Gamma \rightarrow \tilde{\rho}_{H}\left(\Gamma, \Gamma^{\prime}\right)$ ! This sounds like the end of our attempt to warp a shape onto another using an approximation of the Hausdorff distance. But things turn out not to be so bad. First, the existence of a minimum is guaranteed by Proposition 23 which says that $\tilde{\rho}_{H}$ is continuous on $\mathcal{S}$ for the Hausdorff topology, Theorem 12 which says that $\mathcal{D} \mathcal{Z}_{0}$ is compact for this topology, and Proposition 22 which tells us that the l.s.c. extension of $\tilde{\rho}_{H}(\cdot, \Gamma)$ has a minimum in the closure $\overline{\mathcal{S}}$ of $\mathcal{S}$ in $\mathcal{D} \mathcal{Z}_{0}$.

We show in the next section that phenomena like the one described above are for all practical matters "invisible" since confined to an arbitrarily small Hausdorff ball centered at $\Gamma$.

\subsection{Quality of the Approximation $\tilde{\rho}_{H}$ of $\rho_{H}$}

In this section we make more precise the idea that $\tilde{\rho}_{H}$ can be made arbitrarily close to $\rho_{H}$. Because of the form of (23) we seek upper and lower bounds of such quantities as $\langle f\rangle_{\Gamma}^{\psi}$, where $f$ is a continuous real function defined on $\Gamma$. We note by $f_{\max }$ and $f_{\min }$ the maximum and minimum values of $f$ on $\Gamma$.

The expression

$$
\langle f\rangle_{\Gamma}^{\psi}=\psi^{-1}\left(\frac{1}{|\Gamma|} \int_{\Gamma} \psi \circ f\right),
$$

yields, if $\psi$ is strictly increasing,

$$
\langle f\rangle_{\Gamma}^{\psi} \leq \psi^{-1}\left(\frac{1}{|\Gamma|} \int_{\Gamma} \psi \circ f_{\max }\right)=f_{\max } .
$$

and, similarly,

$$
\langle f\rangle_{\Gamma}^{\psi} \geq f_{\min }
$$

If $f \geq f_{\text {moy }}$ on a set $F$ of the curve $\Gamma$, of length $|F|(\leq|\Gamma|)$ :

$$
\begin{aligned}
\langle f\rangle_{\Gamma}^{\psi} & =\psi^{-1}\left(\frac{1}{|\Gamma|} \int_{F} \psi \circ f+\frac{1}{|\Gamma|} \int_{\Gamma \backslash F} \psi \circ f\right) \\
& \geq \psi^{-1}\left(\frac{|F|}{|\Gamma|} \psi \circ f_{\text {moy }}+\frac{|\Gamma|-|F|}{|\Gamma|} \psi \circ f_{\text {min }}\right) \\
& \geq \psi^{-1}\left(\frac{|F|}{|\Gamma|} \psi \circ f_{\text {moy }}\right) .
\end{aligned}
$$

To analyze this lower bound, we introduce the following notation. Given $\Delta, \alpha \geq$ 0 , we note by $\mathcal{P}(\Delta, \alpha)$ the following property:

$$
\mathcal{P}(\Delta, \alpha): \quad \text { for all } x \in \mathbb{R}^{+}, \quad \Delta \psi(x) \geq \psi(\alpha x) .
$$


This property is satisfied for $\psi(x)=x^{\beta}, \beta \geq 0$. The best pairs $(\Delta, \alpha)$ verifying $\mathcal{P}$ are such that $\Delta=\alpha^{\beta}$. In the sequel, we say that a function $\psi$ is admissible for $\mathcal{P}$ if

$$
\text { for all } \Delta \in] 0 ; 1[, \quad \text { there exists } \alpha \in] 0 ; 1[, \quad \mathcal{P}(\Delta, \alpha),
$$

and, conversely,

$$
\text { for all } \alpha \in] 0 ; 1[, \quad \text { there exists } \Delta \in] 0 ; 1[, \quad \mathcal{P}(\Delta, \alpha) .
$$

Let us assume that $\psi$ is admissible and note that we can rewrite $\mathcal{P}(\Delta, \alpha)$,

$$
\text { for all } x \in \mathbb{R}^{+}, \quad \psi^{-1}(\Delta \psi(x)) \geq \alpha x .
$$

For $\Delta=|F| /|\Gamma|$ and $x=f_{\text {moy }}$ we obtain for the largest $\alpha(\Delta)$ the following lower-bound:

$$
\langle f\rangle_{\Gamma}^{\psi} \geq \psi^{-1}\left(\Delta \psi\left(f_{\text {moy }}\right)\right) \geq \alpha f_{\text {moy }} .
$$

In other words, for each arbitrary percentage $\Delta$ there exists an $\alpha$ such that if $\left|\left\{f \geq f_{\text {moy }}\right\}\right| \geq \Delta|\Gamma|$, then $\langle f\rangle_{\Gamma}^{\psi} \geq \alpha f_{\text {moy }}$. Conversely, for a given value of $\alpha$, there exists a $\Delta$ such that it is sufficient that $\left|\left\{f \geq f_{\text {moy }}\right\}\right| \geq \Delta|\Gamma|$ to have $\langle f\rangle_{\Gamma}^{\psi} \geq \alpha f_{\text {moy }}$.

For each choice of $(\Delta, \alpha)$, the bracket $\langle f\rangle_{\Gamma}^{\psi}$ acts as a filter which only "looks" at the values of $f$ along $\Gamma$ such that the subset $F$ of $\Gamma$ where they are reached is of relative length $|F| /|\Gamma| \geq \Delta$, meaning that one neglects the "details of relative importance $\leq \Delta$," and that the accuracy of the filter is relative, since it depends upon the product of $\alpha(\leq 1)$ with $f_{\text {moy }}$.

One has even more: The above admissible family of functions $\psi$ allows one to select an arbitrary accuracy, i.e., to choose both $\Delta$ as close as possible to 0 , and $\alpha$ as close as possible to 1 , the best pairs $(\alpha, \Delta)$ for $\psi(x)=x^{\beta}$ satisfying $\Delta=\alpha^{\beta}$, it is sufficient to choose $\beta$ large enough.

Similar properties hold for such brackets as $\langle f\rangle_{\Gamma}^{\varphi}$ where $\varphi$ is strictly decreasing. We have, as in the previous case,

$$
f_{\min } \leq\langle f\rangle_{\Gamma}^{\varphi} \leq f_{\max }
$$

Proceeding as before, if $\left|\left\{f \leq f_{\text {moy }}\right\}\right| \geq \Delta|\Gamma|$ and the pair $(\Delta, \alpha)$ satisfies $\mathcal{P}$ for the function $\varphi$, we obtain

$$
\begin{aligned}
\frac{1}{|\Gamma|} \int_{\Gamma} \varphi \circ f & \geq \Delta \varphi\left(f_{\text {moy }}\right), \\
\langle f\rangle_{\Gamma}^{\varphi} & \leq \varphi^{-1}\left(\Delta \varphi\left(f_{\text {moy }}\right)\right), \\
\langle f\rangle_{\Gamma}^{\varphi} & \leq \alpha f_{\text {moy }} .
\end{aligned}
$$

Admissible functions are $\varphi(x)=x^{-\beta}, \beta>0$; the accuracy increases when $\alpha$ tends to $1^{-}$and $\Delta$ to $0^{+}$; this is always possible by choosing large values of $\beta$, and $\Delta=\alpha^{-\beta}$.

We now have all the ingredients for comparing $\tilde{\rho}_{H}$ and $\rho_{H}$. We start with two definitions. 


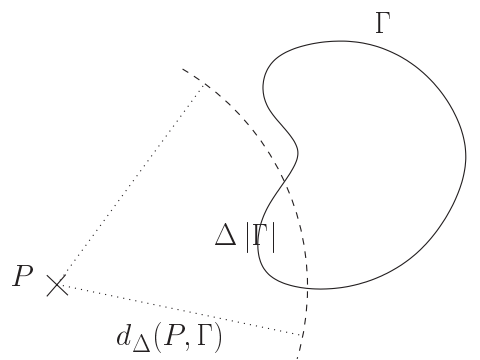

Fig. 8. Geometric interpretation of $d_{\Delta}(P, \Gamma): \Delta$ is the "percentage" of points of $\Gamma$ whose distance to $P$ is less than $d_{\Delta}(P, \Gamma)$.

Definition 28. Let $\Gamma$ be a shape. For each point $P$ of $D$ we note (see Figure 8):

$$
d_{\Delta}(P, \Gamma)=\inf \left\{x \in \mathbb{R}^{+} ;|\{Q \in \Gamma ; d(P, Q) \leq x\}| \geq \Delta|\Gamma|\right\} .
$$

Definition 29. Let $\Gamma$ and $\Gamma^{\prime}$ be two shapes, we define (see Figure 9):

$$
d^{\Delta}\left(\Gamma^{\prime}, \Gamma\right)=\sup \left\{x \in \mathbb{R}^{+} ;\left|\left\{Q \in \Gamma ; d\left(Q, \Gamma^{\prime}\right) \geq x\right\}\right| \geq \Delta|\Gamma|\right\} .
$$

If $\varphi($ resp., $\psi)$ is an admissible function, we note $\left(\Delta_{\varphi}, \alpha_{\varphi}\right)$ (resp., $\left.\left(\Delta_{\psi}, \alpha_{\psi}\right)\right)$ a pair $(\Delta, \alpha)$ for the bracket $\langle\cdot\rangle_{\Gamma}^{\varphi}\left(\operatorname{resp} .,\langle\cdot\rangle_{\Gamma}^{\psi}\right)$.

The following proposition relates $\tilde{\rho}_{H}$ to $d_{\Delta}$ and $d^{\Delta}$ :

Proposition 30. The following relation is satisfied by $\tilde{\rho}_{H}, d_{\Delta}$, and $d^{\Delta}$ :

$$
\begin{aligned}
& \alpha_{\psi} \alpha_{\psi} \max \left(d^{\Delta_{\psi}}\left(\Gamma, \Gamma^{\prime}\right), d^{\Delta_{\psi}}\left(\Gamma^{\prime}, \Gamma\right)\right) \\
& \quad \leq \tilde{\rho}_{H}\left(\Gamma, \Gamma^{\prime}\right) \leq \alpha_{\varphi} \max \left(\sup _{P \in \Gamma^{\prime}} d_{\Delta_{\varphi}}(P, \Gamma), \sup _{P \in \Gamma} d_{\Delta_{\varphi}}\left(P, \Gamma^{\prime}\right)\right) .
\end{aligned}
$$

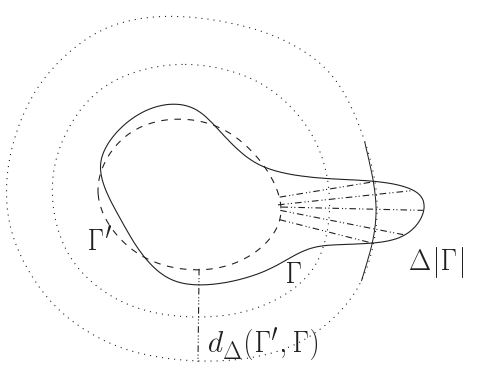

Fig. 9. Geometric interpretation of $d^{\Delta}\left(\Gamma^{\prime}, \Gamma\right): \Delta$ is the "percentage" of points of $\Gamma$ whose distance to $\Gamma^{\prime}$ is greater than $d^{\Delta}\left(\Gamma^{\prime}, \Gamma\right)$. 
Proof. We notice that

$$
\text { for all } P \in \mathbb{R}^{2}, \quad d(P, \Gamma) \leq\langle d(P, \cdot)\rangle_{\Gamma}^{\varphi} \leq \alpha_{\varphi} d_{\Delta_{\varphi}}(P, \Gamma)
$$

and, therefore,

$$
\alpha_{\psi} d^{\Delta_{\psi}}\left(\Gamma, \Gamma^{\prime}\right) \leq\left\langle\langle d(\cdot, \cdot)\rangle_{\Gamma}^{\varphi}\right\rangle_{\Gamma^{\prime}}^{\psi} \leq \alpha_{\varphi} \sup _{P \in \Gamma^{\prime}} d_{\Delta_{\varphi}}(P, \Gamma) .
$$

$\tilde{\rho}_{H}$ is a discrete bracket $\langle\cdot, \cdot\rangle_{\theta}$ of two such terms, $\theta$ an increasing function. We note by $\alpha_{\theta}$ an $\alpha$ associated to $\Delta=\frac{1}{2}$ through $\mathcal{P}$ for $\theta$. For all positive $a$ and $b$ we have

$$
\begin{aligned}
\theta^{-1}\left(\frac{1}{2} \theta(\max (a, b))\right) & \leq\langle a, b\rangle_{\theta} \leq \max (a, b), \\
\alpha_{\theta} \max (a, b) & \leq\langle a, b\rangle_{\theta} \leq \max (a, b)
\end{aligned}
$$

from where the conclusion follows.

We now relate $d_{\Delta}$ and $d^{\Delta}$ to the Hausdorff distance $\rho_{H}$.

Proposition 31. For all $P \in D$ and for all shapes $\Gamma$ and $\Gamma^{\prime}$ we have

$$
d(P, \Gamma) \leq d_{\Delta}(P, \Gamma) \leq d(P, \Gamma)+\frac{\Delta}{2}|\Gamma|,
$$

and

$$
d_{H}\left(\Gamma, \Gamma^{\prime}\right)-\Delta \frac{|\Gamma|+\left|\Gamma^{\prime}\right|}{2} \leq d^{\Delta}\left(\Gamma^{\prime}, \Gamma\right) \leq d_{H}\left(\Gamma, \Gamma^{\prime}\right)+\Delta \frac{|\Gamma|+\left|\Gamma^{\prime}\right|}{2} .
$$

Proof. The lower-bound on $d_{\Delta}(P, \Gamma)$ is easy to obtain, the upper-bound can be obtained by contradiction as follows: let us assume that there exists a point $P$ and a curve $\Gamma$ such that the upper-bound is not satisfied. Hence

$$
d_{\Delta}(P, \Gamma)>d(P, \Gamma)+\frac{\Delta}{2}|\Gamma|,
$$

$\Gamma$ being compact, there exists a point $Q$ of $\Gamma$ such that $d(P, Q)=d(P, \Gamma)$. Let us now consider $\Gamma$ as a $C^{2}$ function from $[0,1]$ to $\mathbb{R}^{2}$ such that $\left|\Gamma^{\prime}(p)\right|=c^{\text {ste }}=|\Gamma|$ for all $p$ 's in $[0,1]$. Let $q \in[0,1]$ be such that $\Gamma(q)=Q$, and consider the image by $\Gamma$ of $I=\{p|| p-q \mid \leq \Delta / 2\}$ (assuming $q \in] \Delta / 2,1-\Delta / 2[$, otherwise the proof can be easily modified). By construction

$$
|\Gamma(I)|=|I||\Gamma|=\Delta|\Gamma|,
$$

and for all points $R$ of $\Gamma(I)$ of parameter $r$,

$$
P R \leq P Q+Q R \leq d(P, A)+|r-q||\Gamma| \leq d(P, A)+\frac{1}{2} \Delta|A| .
$$


We have found a measurable subset of the curve $\Gamma$ of length larger than or equal to $\Delta|\Gamma|$ such that all its points are at a distance of $P$ less than $d(P, \Gamma)+\frac{1}{2} \Delta|\Gamma|$, a contradiction.

The proof of the second set of inequalities proceeds in a similar fashion by considering subsets of the curves $\Gamma$ and $\Gamma^{\prime}$ centered at points $P$ of $\Gamma$ and $Q$ of $\Gamma^{\prime}$ such that $\rho_{H}\left(\Gamma, \Gamma^{\prime}\right)=d(P, Q)$; this is always possible since $\Gamma$ and $\Gamma^{\prime}$ are compact.

By combining Propositions 30 and 31 we obtain

Proposition 32. $\tilde{\rho}_{H}\left(\Gamma, \Gamma^{\prime}\right)$ has the following upper and lower bounds:

$$
\begin{aligned}
& \alpha_{\theta} \alpha_{\psi}\left(\rho_{H}\left(\Gamma, \Gamma^{\prime}\right)-\Delta_{\psi} \frac{|\Gamma|+\left|\Gamma^{\prime}\right|}{2}\right) \\
& \quad \leq \tilde{\rho}_{H}\left(\Gamma, \Gamma^{\prime}\right) \leq \alpha_{\varphi}\left(\rho_{H}\left(\Gamma, \Gamma^{\prime}\right)+\Delta_{\varphi} \frac{|\Gamma|+\left|\Gamma^{\prime}\right|}{2}\right)
\end{aligned}
$$

We can now characterize the shapes $\Gamma$ and $\Gamma^{\prime}$ such that

$$
\tilde{\rho}_{H}\left(\Gamma, \Gamma^{\prime}\right)<\tilde{\rho}_{H}(\Gamma, \Gamma) .
$$

Theorem 33. Condition (32) is equivalent to

$$
\rho_{H}\left(\Gamma, \Gamma^{\prime}\right)<4 c_{0} \Delta
$$

where the constant $c_{0}$ is defined in Definition 7 and Proposition 8, and $\Delta$ in the proof.

Proof. We use the upper- and lower-bounds (31) derived in Proposition 32 and write

$$
\alpha_{\theta} \alpha_{\psi}\left(\rho_{H}\left(\Gamma, \Gamma^{\prime}\right)-\Delta_{\psi} \frac{|\Gamma|+\left|\Gamma^{\prime}\right|}{2}\right)<\alpha_{\varphi} \Delta_{\varphi}|\Gamma| .
$$

To simplify the analysis, let us assume that $\alpha_{\theta} \alpha_{\psi}=\alpha_{\varphi}$ and $\Delta_{\psi}=\Delta_{\varphi}=\Delta$, we obtain

$$
\rho_{H}\left(\Gamma, \Gamma^{\prime}\right)<\left(\frac{3}{2}|\Gamma|+\frac{1}{2}\left|\Gamma^{\prime}\right|\right) \Delta,
$$

and hence (Proposition 8)

$$
\rho_{H}\left(\Gamma, \Gamma^{\prime}\right)<4 c_{0} \Delta
$$

Conversely, if $\Gamma^{\prime}$ is not in the Hausdorff ball with center $\Gamma$ and radius $4 c_{0} \Delta$, we necessarily have $\tilde{\rho}_{H}\left(\Gamma, \Gamma^{\prime}\right)>\tilde{\rho}_{H}(\Gamma, \Gamma)$.

From this we conclude that, since $\Delta$ can be made arbitrarily close to 0 , and the length of shapes is bounded, strange phenomena such as a shape $\Gamma^{\prime}$ closer to a 
shape $\Gamma$ than $\Gamma$ itself (in the sense of $\tilde{\rho}_{H}$ ) cannot occur or rather will be "invisible" to our algorithms.

For completeness we now present an example of such a phenomenon. In detail, we construct a pair $\left(\Gamma_{1}, \Gamma_{2}\right)$ of curves of $\mathcal{S}$ such that $\tilde{\rho}_{H}\left(\Gamma_{1}, \Gamma_{2}\right)<\tilde{\rho}_{H}\left(\Gamma_{1}, \Gamma_{1}\right)$. We assume for simplicity that the function $\theta$ in (23) is the identity. Let $O$ be a point in the plane and consider the family $\left(C_{r}\right), r>0$, of circles of center $O$ and radius $r$. We note $T(r)$ the distance $\tilde{\rho}_{H}\left(C_{r}, C_{r}\right)$ and, for all points $P$, $D(P, r)=\langle d(P, \cdot)\rangle_{C_{r}}^{\varphi}$. Notice that $T(r)=\langle D(\cdot, r)\rangle_{C_{r}}^{\psi}$. For symmetry reasons (rotation invariance) $D(P, r)$ is constant on $C_{r}$, we note by $D(r)$ this value. Hence we have $T(r)=D(r)$. Let us compute $D(r)$ (see Figure 10):

$$
\begin{aligned}
D(r) & =\varphi^{-1}\left(\frac{1}{2 \pi r} \int_{0}^{2 \pi} \varphi\left(2 r \sin \frac{\theta}{2}\right) r d \theta\right) \\
& =\varphi^{-1}\left(\frac{1}{2 \pi} \int_{0}^{2 \pi} \varphi\left(2 r \sin \frac{\theta}{2}\right) d \theta\right) .
\end{aligned}
$$

The function $r \rightarrow r \sin (\theta / 2)$ is strictly increasing for each $0<\theta<2 \pi$, the functions $\varphi$ and $\varphi^{-1}$ are strictly decreasing, hence $r \rightarrow T(r)$ is strictly increasing. In particular, $C_{r}$ is not a local minimum of $\Gamma \rightarrow \tilde{\rho}_{H}(\Gamma, \Gamma)$ and hence not a local minimum of $\Gamma \rightarrow \tilde{\rho}_{H}\left(C_{r}, \Gamma\right)$. Therefore, there exists $\varepsilon>0$ such that $\tilde{\rho}_{H}\left(C_{r}, C_{r-\varepsilon}\right)<\tilde{\rho}_{H}\left(C_{r}, C_{r}\right)$, see Figure 10.

\subsection{Applying the Theory}

In practice, the energy that we minimize is not $\tilde{\rho}_{H}$ but is in fact a "regularized" version obtained by combining $\tilde{\rho}_{H}$ with a term $E_{L}$ which depends upon the lengths of the two curves. A natural candidate for $E_{L}$ is $\max \left(|\Gamma|,\left|\Gamma^{\prime}\right|\right)$ since it acts only if $|\Gamma|$ becomes larger than $\left|\Gamma^{\prime}\right|$, thereby avoiding undesirable oscillations. To obtain smoothness, we approximate the max with a $\Psi$-average:

$$
E_{L}\left(|\Gamma|,\left|\Gamma^{\prime}\right|\right)=\left\langle|\Gamma|,\left|\Gamma^{\prime}\right|\right\rangle^{\Psi} .
$$

We know that the function $\Gamma \rightarrow|\Gamma|$ is in general l.s.c. It is in fact continuous on $\mathcal{S}$ (see the proof of Proposition 23) and takes its values in the interval [0,2 $\left.2 c_{0}\right]$, hence:
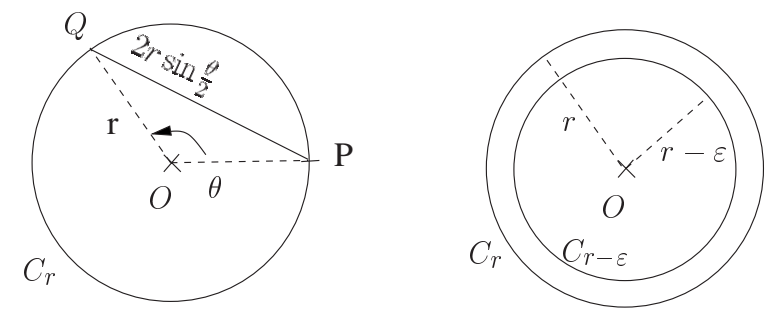

Fig. 10. The curves $C_{r}$ and $C_{r-\varepsilon}$ satisfy $\tilde{\rho}_{H}\left(C_{r}, C_{r-\varepsilon}\right)<\tilde{\rho}_{H}\left(C_{r}, C_{r}\right)$. 
Proposition 34. The function $\mathcal{S} \rightarrow \mathbb{R}^{+}$given by $\Gamma \rightarrow E_{L}\left(\Gamma, \Gamma^{\prime}\right)$ is continuous for the Hausdorff topology.

Proof. This is clear since $E_{L}$ is a combination of continuous functions.

We combine $E_{L}$ with $\tilde{\rho}_{H}$ the expected way, i.e., by computing their $\tilde{\Psi}$ average so that the final energy is

$$
E\left(\Gamma, \Gamma^{\prime}\right)=\left\langle\tilde{\rho}_{H}\left(\Gamma, \Gamma^{\prime}\right), E_{L}\left(|\Gamma|,\left|\Gamma^{\prime}\right|\right)\right\rangle^{\tilde{\Psi}} .
$$

The function $E: \mathcal{S} \times \mathcal{S} \rightarrow \mathbb{R}^{+}$is continuous for the Hausdorff metric because of Propositions 23 and 34 and therefore

Proposition 35. The function $\Gamma \rightarrow E\left(\Gamma, \Gamma^{\prime}\right)$ defined on the set of shapes $\mathcal{S}$ has at least a minimum in the closure $\overline{\mathcal{S}}$ of $\mathcal{S}$ in $\mathcal{L}_{0}$.

Proof. This is a direct application of Proposition 22 applied to the function $E$

We call the resulting warping technique the Hausdorff warping. A first example, the Hausdorff warping of two circles, is shown in Figure 11. A second example, the Hausdorff warping of two hand silhouettes, is shown in Figure 12

We have borrowed the example in Figure 13 from the database (www.ee. surrey.ac.uk/Research/VSSP/imagedb/demo.html) of fish silhouettes collected by the researchers of the University of Surrey at the center for Vision, Speech, and Signal Processing (www. ee. surrey.ac.uk/Research/VSSP). This database contains 1100 silhouettes. A few steps of the result of Hausdorff warping one of these silhouettes onto another are shown in Figure 13. Another similar example is shown in Figure 14. Note that, prior to warping, the two shapes

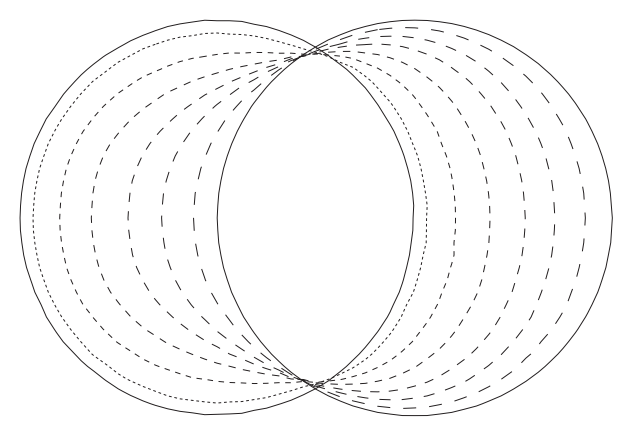

Fig. 11. The result of the Hausdorff warping of two circles. The two circles are represented in continuous line while the intermediate shapes are represented in dotted lines. 


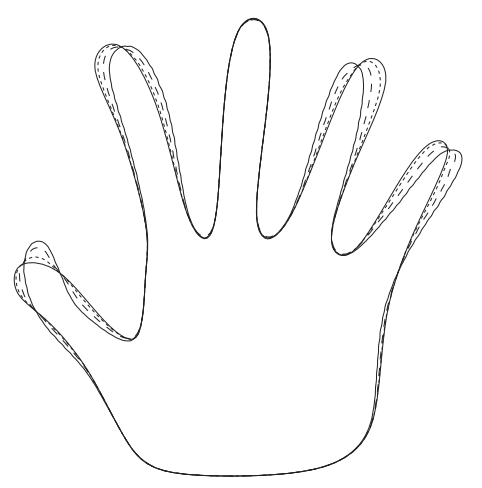

Fig. 12. The result of the Hausdorff warping of two hand silhouettes. The two hands are represented in continuous line while the intermediate shapes are represented in dotted lines.

have been normalized in such a way as to align their centers of gravity and their principal axes.

Figures 15 and 16 give a better understanding of the behavior of Hausdorff warping. A slightly displaced detail "warps back" to its original place (Figure 15). Displaced further, the same detail is considered as another one and disappears during the warping process while the original one reappears (Figure 16).

Finally, Figure 17 shows the Hausdorff warping of an open curve to another.

Note also that other warpings are given by the minimization of other approximations of the Hausdorff distance. Figure 18 shows the warping of a rough curve to the silhouette of a fish and bubbles given by the minimization of the $W^{1,2}$ norm as explained in Section 5.6. Our "level sets" implementation (see Section 8) can deal with the splitting of the source curve while warping onto the target one.
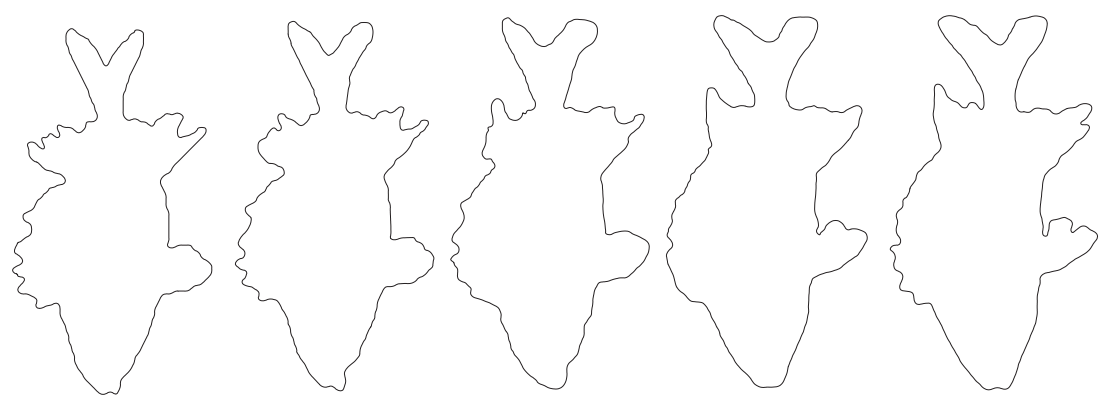

Fig. 13. Hausdorff warping a fish onto another. 


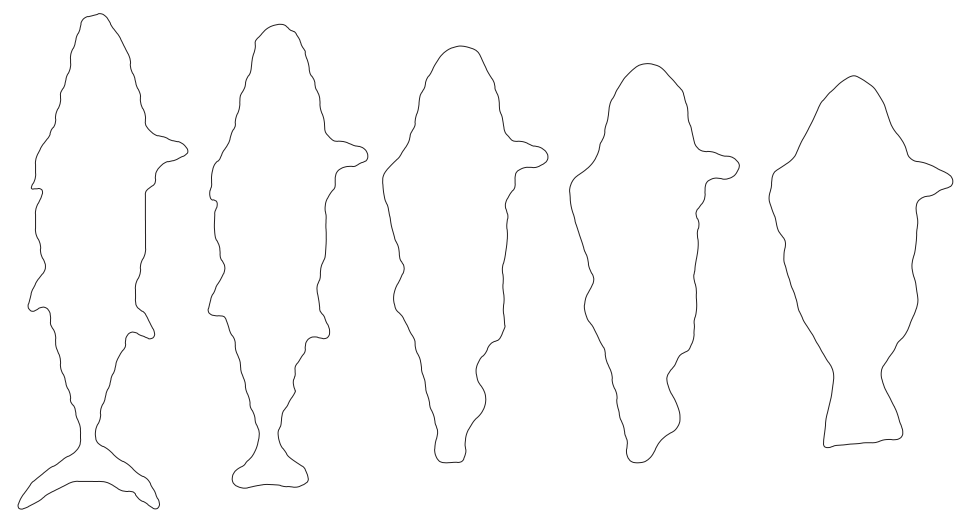

Fig. 14. Another example of fish Hausdorff warping.
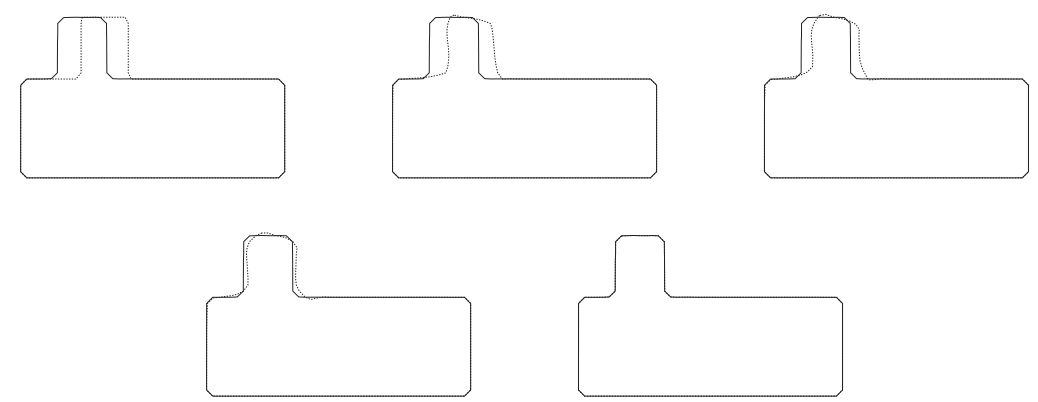

Fig. 15. Hausdorff warping boxes (i). A translation-like behavior.
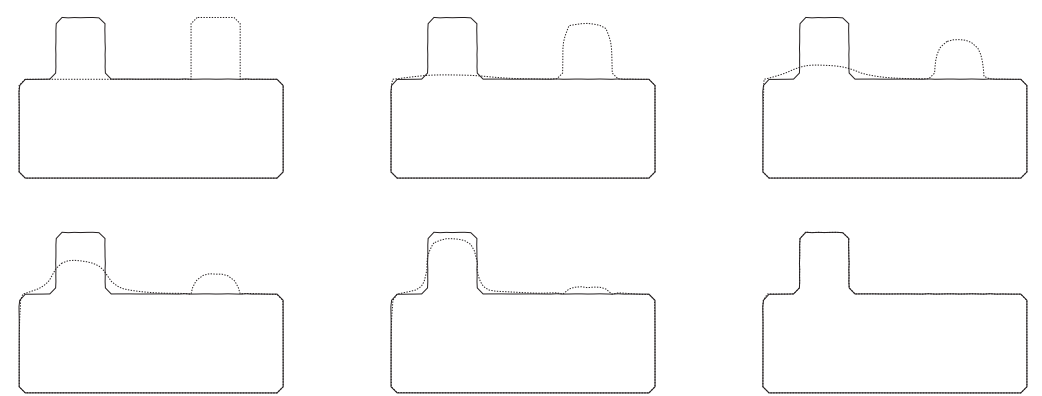

Fig. 16. Hausdorff warping boxes (ii). A different behavior: a detail disappears while another one appears. 


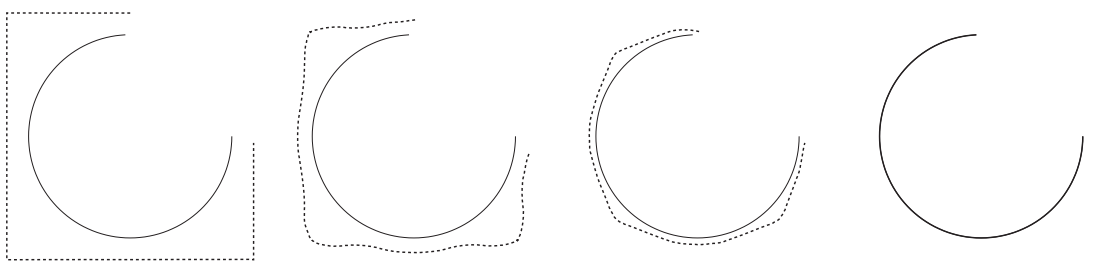

Fig. 17. Hausdorff warping an open curve to another one.

\section{Application to the Computation of the Empirical Mean and Covariance of a Set of Shape Examples}

We have now developed the tools for defining several concepts relevant to a theory of stochastic shapes as well as providing the means for their effective computation. They are based on the use of the function $E$ defined by (34).

\subsection{Empirical Mean}

The first one is that of the mean of a set of shapes. Inspired by the work of Fréchet [17], [18], Karcher [25], W. Kendall [28], and Pennec [38], we provide the following (classical):

Definition 36. Given $\Gamma_{1}, \ldots, \Gamma_{N}, N$ shapes, we define their empirical mean as any shape $\hat{\Gamma}$ that achieves a local minimimum of the function $\mu: \mathcal{S} \rightarrow \mathbb{R}^{+}$defined by

$$
\Gamma \rightarrow \mu\left(\Gamma, \Gamma_{1}, \ldots, \Gamma_{N}\right)=\frac{1}{N} \sum_{i=1, \ldots, N} E^{2}\left(\Gamma, \Gamma_{i}\right) .
$$

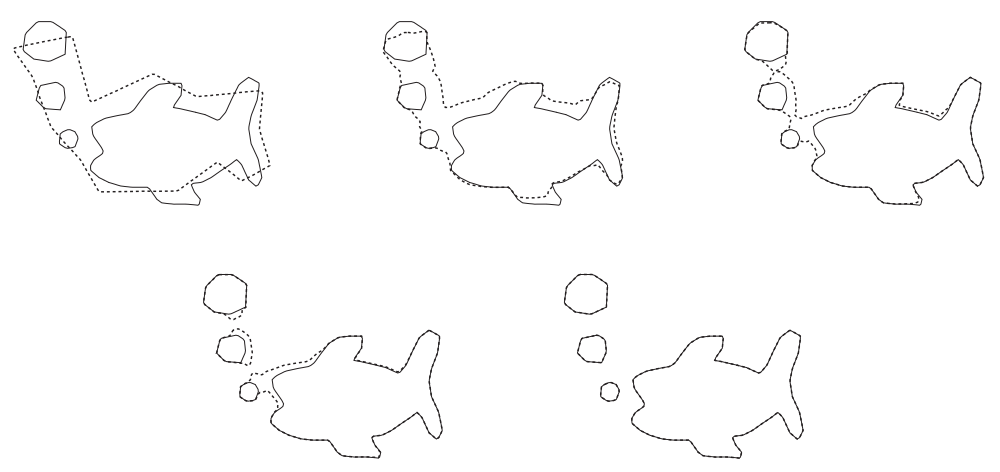

Fig. 18. Splitting while warping. 


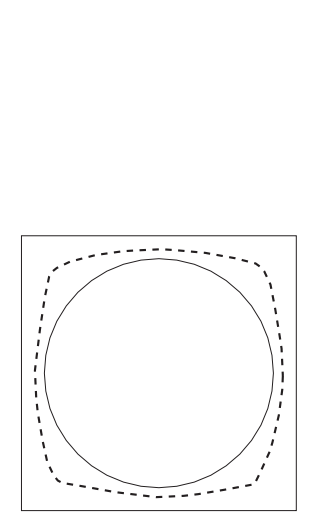

(a)

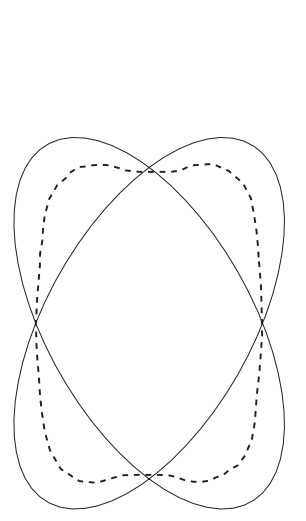

(b)

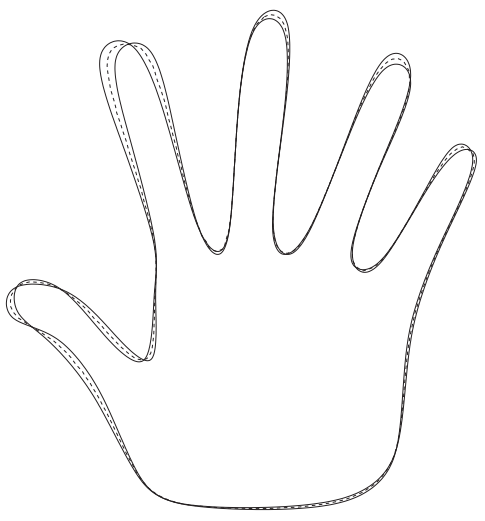

(c)

Fig. 19. Examples of means of several curves: (a) a square and a circle, (b) two ellipses, and (c) two hands.

Note that there may exist several means. We know from Proposition 35 that there exists at least one. An algorithm for computing approximations to an empirical mean of $N$ shapes readily follows from the previous section: start from an initial shape $\Gamma_{0}$ and solve the PDE,

$$
\begin{aligned}
\Gamma_{t} & =-\nabla \mu\left(\Gamma, \Gamma_{1}, \ldots, \Gamma_{N}\right) \mathbf{n}, \\
\Gamma(0, \cdot) & =\Gamma_{0}(\cdot)
\end{aligned}
$$

We show some examples of means computed by this algorithm in Figure 19.

When the number of shapes grows larger, the question of the local minima of $\mu$ becomes a problem (see Figure 20) and the choice of $\Gamma_{0}$ in (35) is an important issue. We have not explored these questions in great detail but observed that the following heuristics led to "visually satisfying" results.

Suppose that the example shapes are given in some order, according to the way they are indexed from 1 to $N$. Initialize $\hat{\Gamma}^{(1)}$ to $\Gamma_{1}$, solve

$$
\begin{aligned}
\Gamma_{t}^{(i+1)} & =-\nabla \mu\left(\Gamma, i \hat{\Gamma}^{(i)}, \Gamma_{i}\right) \mathbf{n}, \\
\Gamma^{(i+1)}(0, \cdot) & =\hat{\Gamma}^{i}(\cdot),
\end{aligned}
$$

and choose $\hat{\Gamma}^{(i+1)}=\Gamma^{(i+1)}$ at convergence, for $i=1, \ldots, N-1$. Of course, there is no guarantee that either the result will be independent of the order of presentation (this may or may not be important, depending on the application) or that it will indeed be a local minimum of $\mu\left(\Gamma, \Gamma_{1}, \ldots, \Gamma_{N}\right)$. Another alternative is to solve (35) by choosing $\Gamma_{0}$ to be one of the given shapes.

We show the result of computing the mean of nine hands with this method in Figure 21. 

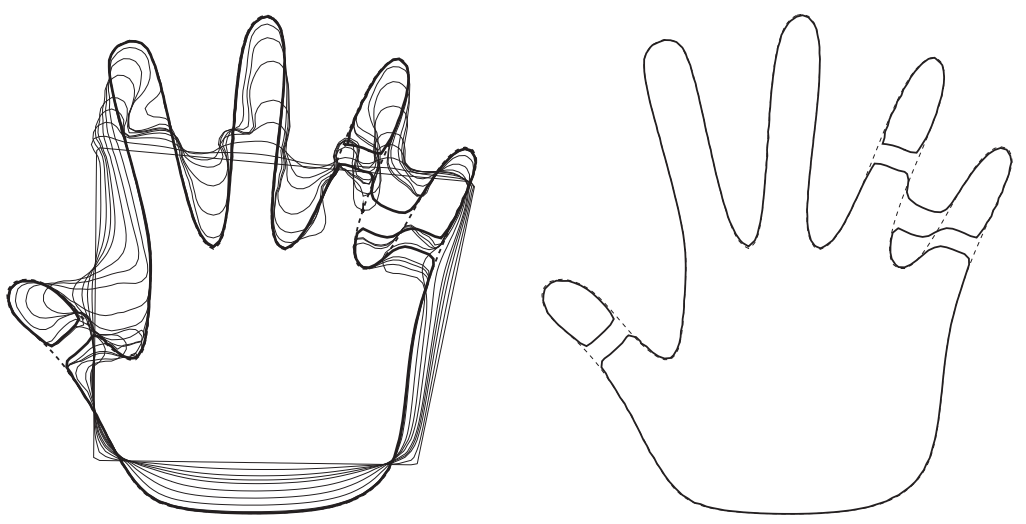

Fig. 20. Example of an evolution which leads to a local minimum: mean of the same nine hands obtained by initializing with another curve (the bold quadrilateral, the expected mean being the dashed curve).

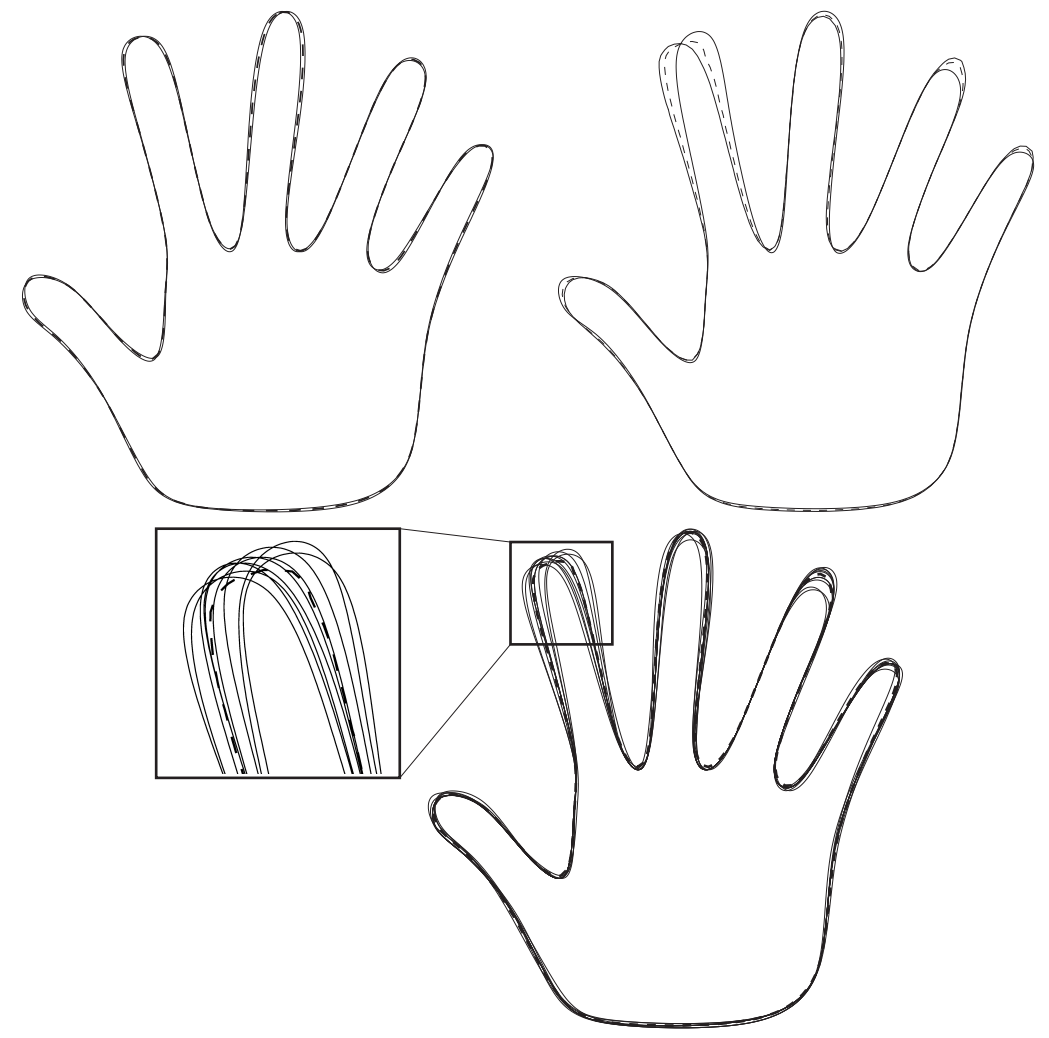

Fig. 21. The mean (the dashed curve) of nine hand silhouettes (the continuous curves) obtained by the sequential suboptimal method described in the text: first step (mean of the two first curves), fifth step (weighted mean of the sixth curve and of the mean of the five first curves from the previous step), and final result. 


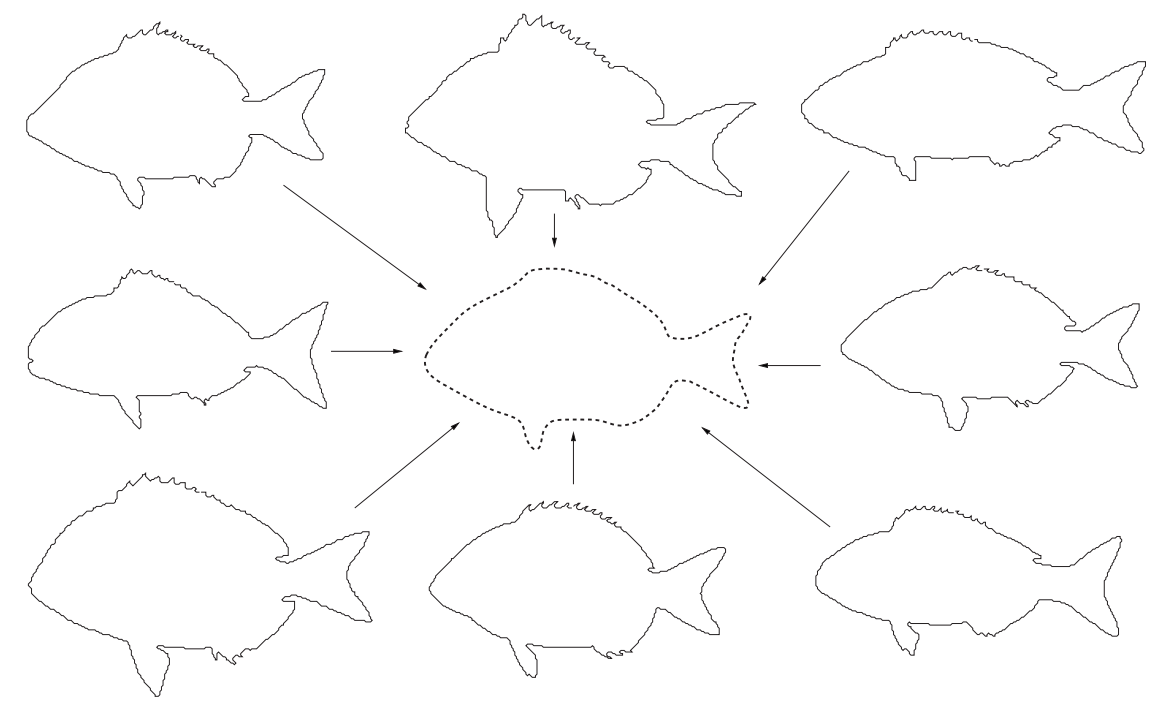

Fig. 22. The mean of eight fish.

Another example of mean is obtained from the previous fish silhouettes database: we have used eight silhouettes, normalized them so that their centers of gravity and principle axes were aligned, and computed their mean, as shown in Figure 22. The initial curve, $\Gamma_{0}$ was chosen to be an enclosing circle.

\subsection{Empirical Covariance}

We can go beyond the definition of the mean and in effect define something similar to the covariance matrix of a set of $N$ shapes.

The function $\mathcal{S} \rightarrow \mathbb{R}^{+}$defined by $\Gamma \rightarrow E^{2}\left(\Gamma, \Gamma_{i}\right)$ has a gradient which defines a normal velocity field, noted $\beta_{i}$, defined on $\Gamma$, such that if we consider the infinitesimal deformation $\Gamma-\beta_{i} \mathbf{n} d \tau$ of $\Gamma$, it decreases the value of $E^{2}\left(\Gamma, \Gamma_{i}\right)$. Each such $\beta_{i}$ belongs to $L^{2}(\Gamma)$, the set of square integrable real functions defined on $\Gamma$. Each $\Gamma_{i}$ defines such a normal velocity field $\beta_{i}$. We consider the mean velocity $\hat{\beta}=(1 / N) \sum_{i=1}^{N} \beta_{i}$ and define the linear operator $\Lambda: L^{2}(\Gamma) \rightarrow L^{2}(\Gamma)$ such that $\beta \rightarrow \sum_{i=1, N}\left\langle\beta, \beta_{i}-\hat{\beta}\right\rangle\left(\beta_{i}-\hat{\beta}\right)$. We have the following:

Definition 37. Given $N$ shapes of $\mathcal{S}$, the covariance operator of these $N$ shapes relative to any shape $\Gamma$ of $\mathcal{S}$ is the linear operator of $L^{2}(\Gamma)$ defined by

$$
\Lambda(\beta)=\sum_{i=1, N}\left\langle\beta, \beta_{i}-\hat{\beta}\right\rangle\left(\beta_{i}-\hat{\beta}\right),
$$

where the $\beta_{i}$ are defined as above, relative to the shape $\Gamma$. 
This operator has some interesting properties which we study next.

Proposition 38. The operator $\Lambda$ is a continuous mapping of $L^{2}(\Gamma)$ into $L^{2}(\Gamma)$.

Proof. We have $\left\|\sum_{i=1, N}\left\langle\beta, \beta_{i}-\hat{\beta}\right\rangle\left(\beta_{i}-\hat{\beta}\right)\right\|_{2} \leq \sum_{i=1, N}\left|\left\langle\beta, \beta_{i}-\hat{\beta}\right\rangle\right| \| \beta_{i}-$ $\hat{\beta} \|_{2}$ and, because of the Schwarz inequality, $\left|\left\langle\beta, \beta_{i}-\hat{\beta}\right\rangle\right| \leq\|\beta\|_{2}\left\|\beta_{i}-\hat{\beta}\right\|_{2}$. This implies that $\left\|\sum_{i=1, N}\left\langle\beta, \beta_{i}-\hat{\beta}\right\rangle\left(\beta_{i}-\hat{\beta}\right)\right\|_{2} \leq K\|\beta\|_{2}$ with $K=\sum_{i=1, N} \| \beta_{i}-$ $\hat{\beta} \|_{2}^{2}$.

$\Lambda$ is in effect a mapping from $L^{2}(\Gamma)$ into its Hilbert subspace $A(\Gamma)$ generated by the $N$ functions $\beta_{i}-\hat{\beta}$. Note that if $\Gamma$ is one of the empirical means of the shapes $\Gamma_{i}$, by definition we have $\hat{\beta}=0$.

This operator acts on what can be thought of as the tangent space to the manifold of all shapes at the point $\Gamma$. We then have

Proposition 39. The covariance operator is symmetric positive definite.

Proof. This follows from the fact that $\langle\Lambda(\beta), \beta\rangle=\langle\beta, \Lambda(\beta)\rangle=\sum_{i=1, N}\langle\beta$, $\left.\beta_{i}-\hat{\beta}\right\rangle^{2}$.

It is also instructive to look at the eigenvalues and eigenvectors of $\Lambda$. For this purpose we introduce the $N \times N$ matrix $\hat{\Lambda}$ defined by $\hat{\Lambda}_{i j}=\left\langle\beta_{i}-\hat{\beta}, \beta_{j}-\hat{\beta}\right\rangle$. We have

Proposition 40. The $N \times N$ matrix $\hat{\Lambda}$ is symmetric semipositive definite. Let $p \leq N$ be its rank, $\sigma_{1}^{2} \geq \sigma_{2}^{2} \geq \cdots \geq \sigma_{p}^{2}>0$ its positive eigenvalues, $\mathbf{u}_{1}, \ldots, \mathbf{u}_{N}$ the corresponding eigenvectors. They satisfy

$$
\begin{aligned}
& \mathbf{u}_{i} \cdot \mathbf{u}_{j}=\delta_{i j}, \quad i, j=1, \ldots, N \text {, } \\
& \hat{\Lambda} \mathbf{u}_{i}=\sigma_{i}^{2} \mathbf{u}_{i}, \quad i=1, \ldots, p, \\
& \hat{\Lambda} \mathbf{u}_{i}=0, \quad p+1 \leq i \leq N \text {. }
\end{aligned}
$$

Proof. The matrix $\hat{\Lambda}$ is clearly symmetric. Now let $\alpha=\left[\alpha_{1}, \ldots, \alpha_{N}\right]^{T}$ be a vector of $\mathbb{R}^{N}, \boldsymbol{\alpha}^{T} \hat{\Lambda} \boldsymbol{\alpha}=\|\beta\|_{2}^{2}$, where $\beta=\sum_{i=1}^{N} \alpha_{i}\left(\beta_{i}-\hat{\beta}\right)$. The remainder of the proposition is simply a statement of the existence of an orthonormal basis of eigenvectors for a symmetric matrix of $\mathbb{R}^{N}$.

The $N$-dimensional vectors $\mathbf{u}_{j}, j=1, \ldots, p$, and the $p$ eigenvalues $\sigma_{k}^{2}, k=$ $1, \ldots, p$, define $p$ modes of variation of the shape $\Gamma$. These modes of variation are normal deformation flows which are defined as follows. We note by $u_{i j}, i, j=$ 
$1, \ldots, N$, the $i$ th coordinate of the vector $\mathbf{u}_{j}$ and $v_{j}$ the element of $A(\Gamma)$ defined by

$$
v_{j}=\frac{1}{\sigma_{j}} \sum_{i=1}^{N} u_{i j}\left(\beta_{i}-\hat{\beta}\right) .
$$

In this case $\Gamma=\hat{\Gamma}, \hat{\beta}=0$. We have the proposition:

Proposition 41. The functions $v_{j}, j=1, \ldots, p$, are an orthonormal set of eigenvectors of the operator $\Lambda$ and form a basis of $A(\Gamma)$.

Proof. Let us form the product $\left\langle v_{j}, v_{k}\right\rangle$ :

$$
\begin{aligned}
\left\langle v_{j}, v_{k}\right\rangle & =\frac{1}{\sigma_{j} \sigma_{k}}\left\langle\sum_{l=1}^{N} u_{l j}\left(\beta_{l}-\hat{\beta}\right), \sum_{m=1}^{N} u_{m k}\left(\beta_{m}-\hat{\beta}\right)\right\rangle \\
& =\frac{1}{\sigma_{j} \sigma_{k}} \sum_{l=1}^{N} u_{l j} \sum_{m=1}^{N}\left\langle\beta_{l}-\hat{\beta}, \beta_{m}-\hat{\beta}\right\rangle u_{m k}=\frac{1}{\sigma_{j} \sigma_{k}} \sum_{l=1}^{N} u_{l j}\left(\hat{\Lambda} \mathbf{u}_{k}\right)_{l} \\
& =\frac{1}{\sigma_{j} \sigma_{k}} \mathbf{u}_{j} \cdot\left(\hat{\Lambda} \mathbf{u}_{k}\right) .
\end{aligned}
$$

According to Proposition 40, $\hat{\Lambda} \mathbf{u}_{k}=\sigma_{k}^{2} \mathbf{u}_{k}$ and $\mathbf{u}_{j} \cdot \mathbf{u}_{k}=\delta_{j k}$, which proves the orthonormality and therefore the linear independence. There remains to show that they generate the whole of $A(\Gamma)$. In order to see this, we consider the element $\beta=\sum_{i=1}^{N} \alpha_{i}\left(\beta_{i}-\hat{\beta}\right)$ of $A(\Gamma)$ and look for the coefficients $\mu_{k}, k=1, \ldots, p$, such that

$$
\sum_{i=1}^{N} \alpha_{i}\left(\beta_{i}-\hat{\beta}\right)=\sum_{k=1}^{p} \mu_{k} v_{k}
$$

We take the Hilbert product of both sides of this equation with $\beta_{j}-\hat{\beta}$ to obtain

$$
(\hat{\Lambda} \boldsymbol{\alpha})_{j}=\sum_{k=1}^{p} \mu_{k}\left\langle v_{k}, \beta_{j}-\hat{\beta}\right\rangle
$$

We then use (36), replace $v_{k}$ with

$$
v_{k}=\frac{1}{\sigma_{k}} \sum_{i=1}^{N} u_{i k}\left(\beta_{i}-\hat{\beta}\right),
$$

and obtain

$$
\left\langle v_{k}, \beta_{j}-\hat{\beta}\right\rangle=\frac{1}{\sigma_{k}} \sum_{i=1}^{N} u_{i k}\left\langle\beta_{i}-\hat{\beta}, \beta_{j}-\hat{\beta}\right\rangle=\frac{1}{\sigma_{k}}\left(\hat{\Lambda} \mathbf{u}_{k}\right)_{j}=\sigma_{k} u_{k j} .
$$


Replacing this value in (38) yields

$$
(\hat{\Lambda} \boldsymbol{\alpha})_{j}=\sum_{k=1}^{p} \mu_{k} \sigma_{k} u_{k j}
$$

or, in matrix form,

$$
\hat{\Lambda} \boldsymbol{\alpha}=\mathbf{U} \operatorname{diag}\left(\sigma_{1}, \ldots, \sigma_{p}\right) \boldsymbol{\mu},
$$

where the $N \times p$ matrix $\mathbf{U}$ is equal to $\left[\mathbf{u}_{1}, \ldots, \mathbf{u}_{p}\right]$. Because the matrix $\mathbf{U}$ satisfies $\mathbf{U}^{T} \mathbf{U}=I_{p}$, the $p \times p$ identity matrix, and $\hat{\Lambda} \mathbf{U}=\mathbf{U} \operatorname{diag}\left(\sigma_{1}^{2}, \ldots, \sigma_{p}^{2}\right)$, we obtain the values of the $\mu_{k}$ :

$$
\operatorname{diag}\left(\sigma_{1}, \ldots, \sigma_{p}\right) \boldsymbol{\mu}=\mathbf{U}^{T} \hat{\Lambda} \boldsymbol{\alpha}=(\hat{\Lambda} \mathbf{U})^{T} \boldsymbol{\alpha}=\left(\mathbf{U} \operatorname{diag}\left(\sigma_{1}^{2}, \ldots, \sigma_{p}^{2}\right)\right)^{T} \boldsymbol{\alpha},
$$

hence

$$
\boldsymbol{\mu}=\operatorname{diag}\left(\sigma_{1}, \ldots, \sigma_{p}\right) \mathbf{U}^{T} \boldsymbol{\alpha} .
$$

Conversely, if we replace the $\mu_{k}$ by these values in the right-hand side of (37), we verify that we obtain the left-hand side.

It remains to verify that $\Lambda\left(v_{j}\right)=\sigma_{j}^{2} v_{j}, j=1, \ldots, p$. By definition

$$
\Lambda\left(v_{j}\right)=\sum_{i=1}^{N}\left\langle v_{j}, \beta_{i}-\hat{\beta}\right\rangle\left(\beta_{i}-\hat{\beta}\right) .
$$

We replace, in the right-hand side of this equation, $v_{j}$ by its expression (36), use Proposition 40, and obtain the desired result.

The velocities $v_{k}, k=1, \ldots, p$, can be interpreted as modes of variation of the shape and the $\sigma_{k}^{2}$,s as variances for these modes. Looking at how the mean shape varies with respect to the $k$ th mode is equivalent to solving the following PDEs:

$$
\Gamma_{t}= \pm v_{k} \mathbf{n}
$$

with initial conditions $\Gamma(0, \cdot)=\hat{\Gamma}(\cdot)$. Note that $v_{k}$ is a function of $\Gamma$ through $\Lambda$ which has to be reevaluated at each time $t$. One usually solves these PDEs until the distance to $\hat{\Gamma}$ becomes equal to $\sigma_{k}$.

An example of this evolution for the case of the fingers is shown in Figure 23. Another interesting case, drawn from the example of the eight fish of Figure 22, is shown in Figure 24 where the first four principal modes of the covariance operator corresponding to those eight sample shapes are displayed.

\section{Some Remarks About Our Implementation}

There are several choices for the definition of $\tilde{\rho}_{H}$ and the implementation of the motion of a curve $\Gamma$ under a velocity field $v: \Gamma_{t}=v$. When $\Gamma$ is composed of one or more closed connected components, we use the level set method introduced by Osher and Sethian in 1987, [36], [41], [35]. 

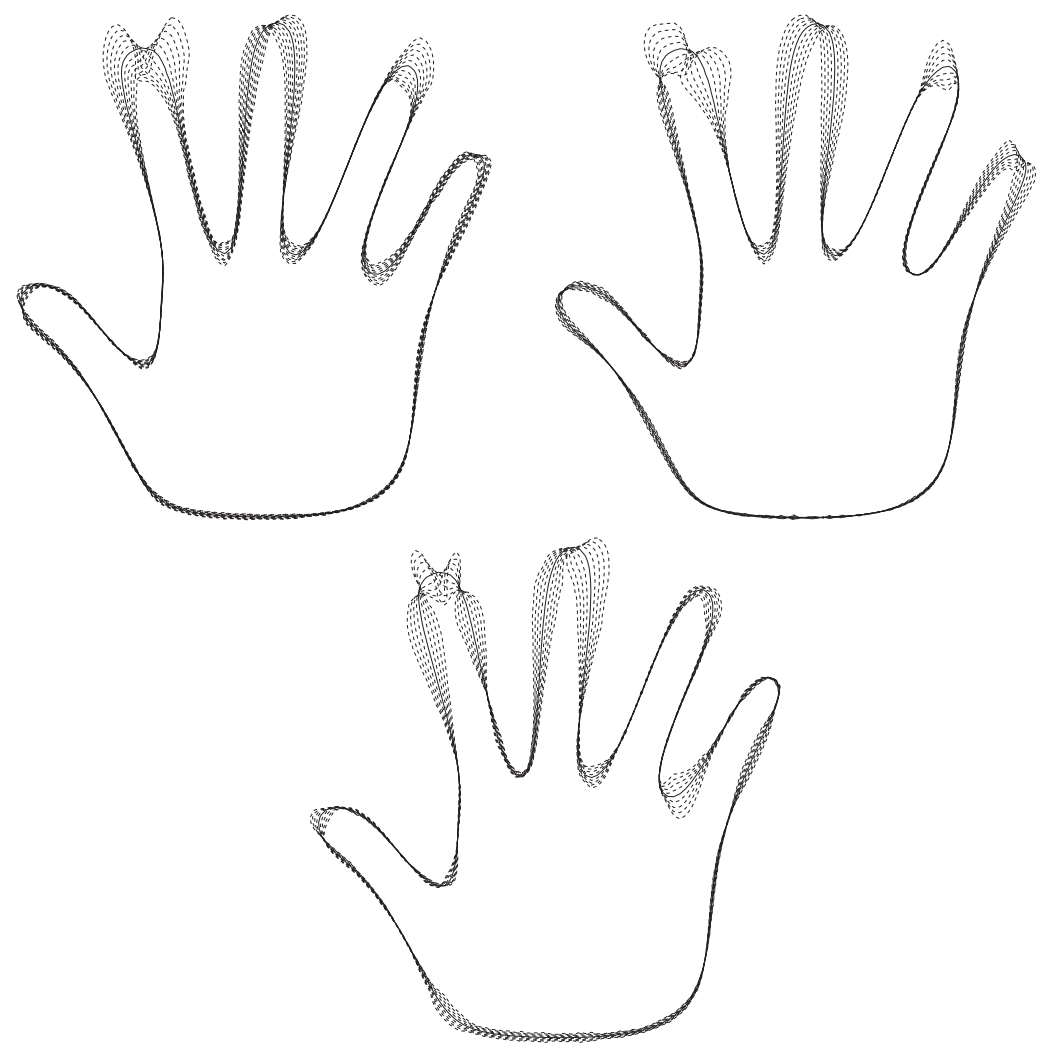

Fig. 23. The first three modes of variation for the nine sample shapes and their mean shown in Figure 21. The mean is shown in thick continuous lines, the solutions of equation (39) for $k=1,2,3$ are represented in dotted lines.

\subsection{The Approximation of the Hausdorff Distance}

We need to decide on the parameters appearing in the definition (18) of $\tilde{\rho}_{H}$, i.e., the values of $\alpha, \beta$, and $\gamma$, and on the function $\varphi \cdot \gamma$ controls how well we approximate the max operator that occurs in the definition of the Hausdorff distance (4). In our implementation we have used the value of 2. The parameters $\alpha$ and $\beta$ control the accuracy with which we approximate the sup and inf operators that appear in equation (4). The higher they are, the better the approximation, but the better the approximation, the higher the numerical difficulties due to the "stiffness" of the function which is of course due the nondifferentiability of the Hausdorff distance. As explained in Section 6.1, the values of $\alpha$ and $\beta$, together with the function $\varphi$, can be ultimately related to the coarseness with which one analyzes the values of the distance function of one curve at points on the other curve, see Figures 8 and 9. In our implementation we use the values $\alpha=\beta=4$ which we found to be a 

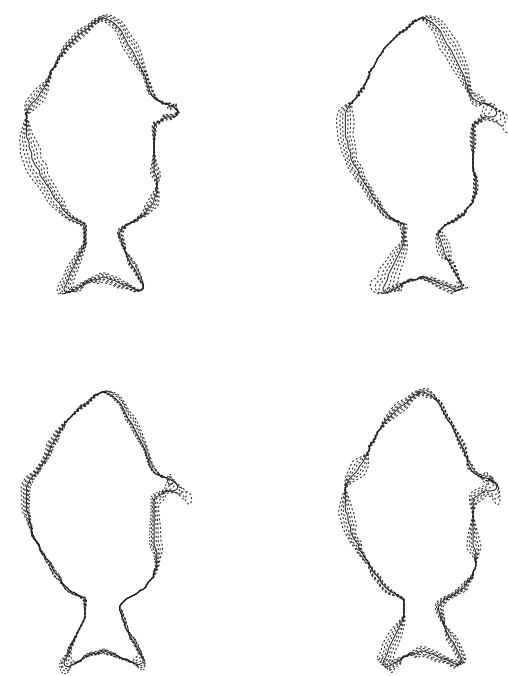

Fig. 24. The first four modes of variation for the eight sample shapes and their mean shown in Figure 22. The mean is shown in thick continuous lines, the solutions of equation (39) for $k=1, \ldots, 4$ are represented in dotted lines.

good compromise between the amount of smoothness of $\tilde{\rho}_{H}$ and the quality of the approximation of $\rho_{H}$. The function $\varphi$ is equal to $\varphi_{1}$ given by equation (17).

\subsection{Closed Curves: The Level Set Method}

The key idea of the level set method is to represent the curve $\Gamma(t)$ implicitly, i.e., as the zero level of some function $u(x, t)$ defined for $x \in D$. Usually, $u$ is negative inside $\Gamma$ and positive outside. It can be easily proved that, if $u$ evolves according to

$$
\frac{\partial u(x, y)}{\partial t}+v \nabla u=0,
$$

then its zero level $\{x \mid \Gamma(x, t)=0\}$ evolves according to the required equation $\Gamma_{t}=v$. Here, $v$ is the desired velocity on $\Gamma$ and is arbitrary elsewhere (see below).

Often, only the normal velocity field $\beta \mathbf{n}$ is important. As $\mathbf{n}=\nabla u /|\nabla u|$, the evolution of $u$ becomes

$$
\frac{\partial u(x, y)}{\partial t}+\beta|\nabla u|=0 .
$$

The advantages of the level set method are well-known: stability, accuracy, convergence to the correct solution, easy extension to higher dimensions, correct handling of topological changes such as breaking and merging. 
An important issue is that $\beta$ is only defined on curve $\Gamma$ in the PDE $\Gamma_{t}=\beta \mathbf{n}$. In many cases, $\beta$ has a natural extension everywhere in domain $D$, so that equation $u_{t}+\beta|\nabla u|=0$ is defined. For instance, when $\beta(x)$ is the curvature of $\Gamma$ at point $x \in \Gamma$, one could choose, at each point $x \in D, \beta(x)$ equal to the curvature of the level set of $u$ going through $x$. In some other cases, like ours, $\beta$ can only be computed on $\Gamma$ and some extension procedure has to be used to get $\beta$ everywhere in $D$. This is now classical [37], [1], [20].

It should be noted that the zero level set of function $u(\cdot, t)$ is only extracted to visualize $\Gamma(t)$, usually with the Marching Cubes algorithm [32] which interpolates its position and gives a nice polygonal approximation of it (a triangulated mesh in three dimensions). For obvious speed and accuracy reasons, it is important not to rely on this approximation to compute the velocity $\beta$. Useful quantities can generally be computed directly from $u$. So it is for the normal and the curvature:

$$
\begin{aligned}
& \mathbf{n}=\nabla u /|\nabla u| \\
& \kappa=-\nabla \cdot(\nabla u /|\nabla u|),
\end{aligned}
$$

and for the integral of some quantity $f(x)$ over $\Gamma$ :

$$
\int_{\Gamma} f(x) d \Gamma(x)=\int_{D} f(x) \delta(u(x))|\nabla u| d x
$$

where $\delta(\cdot)$ is a one-dimensional Dirac function. Our $\beta$ also involves the distance function to the curve $\Gamma$ which has to be known without extracting the zero level set of function $u$. Usually, one takes the signed distance to the initial curve $\Gamma(0)$ as an initial value of $u$. Thus, $u(\cdot, 0)$ can be used to compute $\beta$ at time $t=0$. Yet, $u(\cdot, t)$ has no reason to maintain the distance to $\Gamma(t)$. . except in some implementations where $\beta$ is extended in such a way that the distance function is preserved during the evolution of $u$ (see [20]): this is exactly what we need. And what we use!

In conclusion, in the case of closed curves, the Hausdorff warping (Section 6) and the shape statistics (Section 7) are implemented with a level set method with: (i) velocity extension; (ii) distance function preserving; and (iii) no need to extract the zero level set (except for visualization).

The minimization of the $W^{1,2}$ norm (Section 5.6 and Figure 18) is also implemented with the level set method. As already mentioned, the reprojection on the set of distance functions is a "standard" level set technique [44].

\subsection{Open Curves}

For open curves (Figure 17), the level set method cannot be used. A straight Lagrangian approach and polygonal approximations of the curves were used as a first step toward more refined methods like the ones described in [3]. 


\section{Further Comparison with Other Approaches and Conclusion}

We have presented in Section 1 the similarities and dissimilarities of our work with that of others. We would like to add to this presentation the fact that ours is an attempt to generalize to a nonlinear setting the work that has been done in a linear setting by such scientists as Cootes et al. [8] and by Leventon et al. [30] who, like us, proposed to use distance functions to represent shapes in a statistical framework but used a first-order approximation by assuming that the set of distance functions was a linear manifold [31], [30] which, of course, it is not. Our work shows that dropping the incorrect linearity assumption is possible at reasonable costs, both theoretical and computational. Comparison of results obtained in the two frameworks is a matter of future work.

In this respect we would also like to emphasize that in our framework the process of linear averaging shape representations has been more or less replaced by the linear averaging of the normal deformation fields which are tangent vectors to the manifold of all shapes (see the definition of the covariance operator in Section 7.2) and by solving a PDE based on these normal deformation fields (see the definition of a mean in Section 7.1 and of the deformation modes in Section 7.2).

It is also interesting to recall the fact that our approach can be seen as the opposite of that consisting of first building a Riemannian structure on the set of shapes, i.e., going from an infinitesimal metric structure to a global one. The infinitesimal structure is defined by an inner product in the tangent space (the set of normal deformation fields) and has to vary continuously from point to point, i.e., from shape to shape. As mentioned before, this is mostly dealt with in the work of Miller et al. [34], [49], [52]. The problem with these approaches, beside that of having to deal with parametrizations of the shapes, is that there exist global metric structures on the set of shapes (see Section 2.2) which are useful and relevant to the problem of the comparison of shapes but that do not arise from an infinitesimal structure.

Our approach can be seen as taking the problem from exactly the opposite viewpoint from the previous one: we start with a global metric on the set of shapes $\left(\rho_{H}\right.$ or the $W^{1,2}$ metric) and build smooth functions (in effect smooth approximations of these metrics) that we use as dissimilarity measures or energy functions and minimize using techniques of the calculus of variations by computing their gradient and performing infinitesimal gradient descent. We have seen that in order to compute the gradients we need to define an inner-product of normal deformation flows and the choice of this inner-product may influence the way our algorithms evolve from one shape to another. This last point is related to, but different from, the choice of the Riemaniann metric in the first approach. Its investigation is also a topic of future work.

The question of local minima is, as always, important. We have mentioned that we have found, when computing the mean of a number of shapes, that our warping was sometimes prone to local minima. This is of course not in contradiction with the fact that our energy is defined globally rather than locally. Given $n$ shapes there 
are in general many shapes that minimize the function defined in Definition 36 and the larger $n$ the larger the number of such shapes, in general. This is not counterintuitive. In fact one may argue, as we do above, that the result of the process of learning an average shape by being shown samples of their distribution should depend upon the order of presentation of the examples. The problem of comparing the behavior of our approach with others, using locally defined metrics, is interesting and important, and another topic of future work.

Another advantage of our viewpoint is that it apparently extends graciously to higher dimensions thanks to the fact that we do not rely on parametrizations of the shapes and work intrinsically with their distance functions (or approximations thereof). This is clearly also worth pursuing in future work.

Appendix A. Computation of $\nabla \tilde{\rho}_{H}\left(\Gamma, \Gamma_{0}\right)$

We prove Proposition 25.

Proof. We make a few definitions to simplify notations:

$$
\begin{aligned}
& m_{\Gamma_{0}, \Gamma}^{\varphi, \psi}=\left\langle\langle d(\cdot, \cdot)\rangle_{\Gamma_{0}}^{\varphi}\right\rangle_{\Gamma}^{\psi}, \\
& m_{\Gamma, \Gamma_{0}}^{\varphi, \psi}=\left\langle\langle d(\cdot, \cdot)\rangle_{\Gamma}^{\varphi}\right\rangle_{\Gamma_{0}}^{\psi} .
\end{aligned}
$$

We also define the corresponding functions

$$
\begin{aligned}
m_{\Gamma}^{\varphi}(x)=\langle d(x, \cdot)\rangle_{\Gamma}^{\varphi}, & & m_{\Gamma}^{\psi}(x)=\langle d(x, \cdot)\rangle_{\Gamma}^{\psi}, \\
m_{\Gamma_{0}}^{\varphi}(x)=\langle d(x, \cdot)\rangle_{\Gamma_{0}}^{\varphi}, & & m_{\Gamma_{0}}^{\psi}(x)=\langle d(x, \cdot)\rangle_{\Gamma_{0}}^{\psi} .
\end{aligned}
$$

We then proceed with

$$
\begin{aligned}
& \mathcal{G}\left(\tilde{\rho}_{H}\left(\Gamma, \Gamma_{0}\right), \boldsymbol{\beta}\right)=\frac{1}{2 \theta^{\prime}\left(\tilde{\rho}_{H}\left(\Gamma, \Gamma_{0}\right)\right)}\left[\theta^{\prime}\left(m_{\Gamma_{0}, \Gamma}^{\varphi, \psi}\right) \quad \mathcal{G}\left(m_{\Gamma_{0}, \Gamma}^{\varphi, \psi}, \boldsymbol{\beta}\right)\right. \\
& \left.+\theta^{\prime}\left(m_{\Gamma, \Gamma_{0}}^{\varphi, \psi}\right) \mathcal{G}\left(m_{\Gamma, \Gamma_{0}}^{\varphi, \psi}, \boldsymbol{\beta}\right)\right],
\end{aligned}
$$

because of (13).

Computation of the First Term $\mathcal{G}\left(m_{\Gamma_{0}, \Gamma}^{\varphi, \psi}, \boldsymbol{\beta}\right)$

We apply the chain rule and (12) to obtain

$$
\begin{aligned}
\mathcal{G}\left(m_{\Gamma_{0}, \Gamma}^{\varphi, \psi}, \boldsymbol{\beta}\right)=\frac{1}{\psi^{\prime}\left(m_{\Gamma_{0}, \Gamma}^{\varphi, \psi}\right)} & \frac{1}{|\Gamma|} \mathcal{G}\left(\int_{\Gamma} \psi\left(\langle d(\cdot, \cdot)\rangle_{\Gamma_{0}}^{\varphi}\right), \boldsymbol{\beta}\right) \\
& \left.+\left(\int_{\Gamma} \psi\left(\langle d(\cdot, \cdot)\rangle_{\Gamma_{0}}^{\varphi}\right)\right) \mathcal{G}\left(\frac{1}{|\Gamma|}, \boldsymbol{\beta}\right)\right] .
\end{aligned}
$$


We now compute $\mathcal{G}(1 /|\Gamma|, \boldsymbol{\beta})$ :

$$
\begin{aligned}
\mathcal{G}\left(\frac{1}{|\Gamma|}, \boldsymbol{\beta}\right) & =-\frac{1}{|\Gamma|^{2}} \mathcal{G}(|\Gamma|, \boldsymbol{\beta}) \\
& =-\frac{1}{|\Gamma|^{2}} \mathcal{G}\left(\int_{0}^{1}\left|\Gamma^{\prime}(p)\right| d p, \boldsymbol{\beta}\right) \\
& =-\frac{1}{|\Gamma|^{2}} \int_{0}^{1} \frac{\Gamma^{\prime}(p)}{\left|\Gamma^{\prime}(p)\right|} \cdot \boldsymbol{\beta}^{\prime}(p) d p \\
& =\frac{1}{|\Gamma|^{2}} \int_{0}^{1} \kappa(p) \mathbf{n}(p) \cdot \boldsymbol{\beta}(p)\left|\Gamma^{\prime}(p)\right| d p .
\end{aligned}
$$

The last line is obtained by integrating by parts and using the hypothesis that $\boldsymbol{\beta}$ is parallel to $\mathbf{n} . \kappa(p)$ is the curvature of $\Gamma$ at the point $\Gamma(p)$. This yields

$$
\left(\int_{\Gamma} \psi\left(\langle d(\cdot, \cdot)\rangle_{\Gamma_{0}}^{\varphi}\right)\right) \mathcal{G}\left(\frac{1}{|\Gamma|}, \boldsymbol{\beta}\right)=\frac{1}{|\Gamma|} \psi\left(m_{\Gamma_{0}, \Gamma}^{\varphi, \psi}\right) \int_{\Gamma} \kappa(y) \mathbf{n}(y) \cdot \boldsymbol{\beta}(y) d \Gamma(y) .
$$

We continue with

$$
\begin{aligned}
&\left.\mathcal{G}\left(\int_{\Gamma} \psi(\langle d(\cdot, \cdot))\rangle_{\Gamma_{0}}^{\varphi}\right), \boldsymbol{\beta}\right) \\
&= \mathcal{G}\left(\int_{0}^{1} \psi\left(\langle d(\Gamma(p), \cdot)\rangle_{\Gamma_{0}}^{\varphi}\right)\left|\Gamma^{\prime}(p)\right| d p, \boldsymbol{\beta}\right) \\
&= \int_{0}^{1} \frac{\psi^{\prime}}{\varphi^{\prime}}\left\langle(d(\Gamma(p), \cdot)\rangle_{\Gamma_{0}}^{\varphi}\right) \frac{1}{\left|\Gamma_{0}\right|} \\
& \times \lim _{\tau \rightarrow 0} \frac{\int_{\Gamma_{0}}(\varphi(d(\Gamma(p)+\tau \boldsymbol{\beta}(p), \cdot)-\varphi(d(\Gamma(p), \cdot)))}{\tau}\left|\Gamma^{\prime}(p)\right| d p \\
&+\int_{0}^{1} \psi\left(\langle d(\Gamma(p), \cdot)\rangle_{\Gamma_{0}}^{\varphi}\right) \lim _{\tau \rightarrow 0} \frac{\left|\Gamma^{\prime}(p)+\tau \boldsymbol{\beta}^{\prime}(p)\right|-\left|\Gamma^{\prime}(p)\right|}{\tau} d p .
\end{aligned}
$$

The last term is equal to (using the hypothesis that $\boldsymbol{\beta}(p)$ is parallel to $\mathbf{n}(p)$ for all $p$ 's):

$$
-\int_{0}^{1} \psi\left(\langle d(\Gamma(p), \cdot)\rangle_{\Gamma_{0}}^{\varphi}\right) \kappa(p) \mathbf{n}(p) \cdot \boldsymbol{\beta}(p)\left|\Gamma^{\prime}(p)\right| d p .
$$

The first term can be written

$$
\begin{aligned}
& \int_{0}^{1} \frac{\psi^{\prime}}{\varphi^{\prime}}\left(\langle d(\Gamma(p), \cdot)\rangle_{\Gamma_{0}}^{\varphi}\right) \frac{1}{\left|\Gamma_{0}\right|} \\
& \quad \times\left(\int_{\Gamma_{0}} \varphi^{\prime}(d(\Gamma(p), x)) \frac{\Gamma(p)-x}{d(\Gamma(p), x)} \cdot \boldsymbol{\beta}(p) d \Gamma_{0}(x)\right)\left|\Gamma^{\prime}(p)\right| d p .
\end{aligned}
$$


Combining them we obtain

$$
\int_{\Gamma}\left(\frac{\psi^{\prime}}{\varphi^{\prime}}\left(m_{\Gamma_{0}}^{\varphi}(y)\right)\left\langle\varphi^{\prime}(d(y, \cdot)) \frac{y-\cdot}{d(y, \cdot)}\right\rangle_{\Gamma_{0}}-\psi\left(m_{\Gamma_{0}}^{\varphi}(y)\right) \kappa(y) \mathbf{n}(y)\right) \cdot \boldsymbol{\beta}(y) d \Gamma(y) .
$$

We finally combine (40) and (41),

$$
\begin{aligned}
\mathcal{G}\left(m_{\Gamma_{0}, \Gamma}^{\varphi, \psi}, \boldsymbol{\beta}\right)= & \frac{1}{\psi^{\prime}\left(m_{\Gamma_{0}, \Gamma}^{\varphi, \psi}\right)|\Gamma|} \int_{\Gamma}\left(\frac{\psi^{\prime}}{\varphi^{\prime}}\left(m_{\Gamma_{0}}^{\varphi}(y)\right)\left\langle\varphi^{\prime}(d(y, \cdot)) \frac{y-\cdot}{d(y, \cdot)}\right)_{\Gamma_{0}}\right. \\
& \left.+\left(\psi\left(m_{\Gamma_{0}, \Gamma}^{\varphi, \psi}\right)-\psi\left(m_{\Gamma_{0}}^{\varphi}(y)\right)\right) \kappa(y) \mathbf{n}(y)\right) \cdot \boldsymbol{\beta}(y) d \Gamma(y) .
\end{aligned}
$$

Computation of the Second Term $\mathcal{G}\left(m_{\Gamma, \Gamma_{0}}^{\varphi, \psi}, \boldsymbol{\beta}\right)$

Because of (12) we can write

$$
\begin{aligned}
\mathcal{G}\left(m_{\Gamma, \Gamma_{0}}^{\varphi, \psi}, \boldsymbol{\beta}\right)= & \frac{1}{\psi^{\prime}\left(m_{\Gamma, \Gamma_{0}}^{\varphi, \psi}\right)} \frac{1}{\left|\Gamma_{0}\right|} \int_{\Gamma_{0}} \frac{\psi^{\prime}}{\varphi^{\prime}}\left(\langle d(x, \cdot)\rangle_{\Gamma}^{\varphi}\right) \mathcal{G} \\
& \times\left(\frac{1}{|\Gamma|} \int_{0}^{1} \varphi(d(x, \Gamma(p)))\left|\Gamma^{\prime}(p)\right| d p, \boldsymbol{\beta}\right) d \Gamma_{0}(x)
\end{aligned}
$$

Using the chain rule

$$
\begin{aligned}
\mathcal{G}\left(\frac{1}{|\Gamma|} \int_{0}^{1} \varphi(\right. & \left.d(x, \Gamma(p)))\left|\Gamma^{\prime}(p)\right| d p, \boldsymbol{\beta}\right) \\
= & \left(\int_{\Gamma} \varphi \circ d(x, \cdot)\right) \frac{1}{|\Gamma|^{2}} \int_{0}^{1} \kappa(p) \mathbf{n}(p) \cdot \boldsymbol{\beta}(p)\left|\Gamma^{\prime}(p)\right| d p \\
& +\frac{1}{|\Gamma|} \int_{0}^{1} \varphi^{\prime}(d(\Gamma(p), x)) \frac{\Gamma(p)-x}{d(\Gamma(p), x)} \cdot \boldsymbol{\beta}(p)\left|\Gamma^{\prime}(p)\right| d p \\
& +\frac{1}{|\Gamma|} \int_{0}^{1} \varphi(d(x, \Gamma(p))) \frac{\Gamma^{\prime}(p)}{\left|\Gamma^{\prime}(p)\right|} \cdot \boldsymbol{\beta}^{\prime}(p) d p
\end{aligned}
$$

Under the same hypothesis for $\boldsymbol{\beta}$, the last term is equal to

$$
-\frac{1}{|\Gamma|} \int_{0}^{1} \varphi(d(\Gamma(p), x)) \kappa(p) \mathbf{n}(p) \cdot \boldsymbol{\beta}(p)\left|\Gamma^{\prime}(p)\right| d p
$$


The expression of $\mathcal{G}\left(\tilde{\rho}_{H}\left(\Gamma, \Gamma_{0}\right), \boldsymbol{\beta}\right)$ is obtained by reordering these terms. This yields

$$
\begin{aligned}
\mathcal{G}\left(\tilde{\rho}_{H}\right. & \left.\left(\Gamma, \Gamma_{0}\right), \boldsymbol{\beta}\right) \\
= & \frac{1}{2 \theta^{\prime}\left(\tilde{\rho}_{H}\left(\Gamma, \Gamma_{0}\right)\right)} \int_{\Gamma}\left[\left[\nu \kappa(p) \int_{\Gamma_{0}} \frac{\psi^{\prime}}{\varphi^{\prime}}\left(\langle d(x, \cdot)\rangle_{\Gamma}^{\varphi}\right)\right.\right. \\
& \times\left[\varphi \circ\langle d(x, \cdot)\rangle_{\Gamma}^{\varphi}-\varphi \circ d(x, y)\right] d \Gamma_{0}(x)+\left|\Gamma_{0}\right| \eta \kappa(p) \\
& \left.\times\left[\psi\left(\left\langle\langle d(\cdot, \cdot)\rangle_{\Gamma_{0}}^{\varphi}\right\rangle_{\Gamma}^{\psi}\right)-\psi\left(\langle d(\cdot, y)\rangle_{\Gamma_{0}}^{\varphi}\right)\right]\right] \mathbf{n}(p)+\int_{\Gamma_{0}} \frac{\varphi^{\prime} \circ d(x, y)}{d(x, y)} \\
& \left.\times\left[v \frac{\psi^{\prime}}{\varphi^{\prime}}\left(\langle d(x, \cdot)\rangle_{\Gamma}^{\varphi}\right)+\eta \frac{\psi^{\prime}}{\varphi^{\prime}}\left(\langle d(\cdot, y)\rangle_{\Gamma_{0}}^{\varphi}\right)\right](y-x) d \Gamma_{0}(x)\right] \cdot \boldsymbol{\beta}(p) d \Gamma(y),
\end{aligned}
$$

where

$$
v=\frac{1}{|\Gamma|\left|\Gamma_{0}\right|} \frac{\theta^{\prime}}{\psi^{\prime}}\left(\left\langle\langle d(\cdot, \cdot)\rangle_{\Gamma}^{\varphi}\right\rangle_{\Gamma_{0}}^{\psi}\right) \quad \text { and } \quad \eta=\frac{1}{|\Gamma|\left|\Gamma_{0}\right|} \frac{\theta^{\prime}}{\psi^{\prime}}\left(\left\langle\langle d(\cdot, \cdot)\rangle_{\Gamma_{0}}^{\varphi}\right\rangle_{\Gamma}^{\psi}\right) .
$$

The gradient $\nabla \tilde{\rho}_{H}\left(\Gamma, \Gamma_{0}\right)$ is obtained by identifying the previous expression as an inner product of normal deformation flows $\int_{\Gamma} \nabla \tilde{\rho}_{H}\left(\Gamma, \Gamma_{0}\right)(y) \beta(y) d \Gamma(y)$,

$$
\begin{aligned}
\nabla \tilde{\rho}_{H} & \left(\Gamma, \Gamma_{0}\right)(y) \\
= & \frac{1}{\theta^{\prime}\left(\tilde{\rho}_{H}\left(\Gamma, \Gamma_{0}\right)\right)} \\
& \times\left[v \kappa(p) \int_{\Gamma_{0}} \frac{\psi^{\prime}}{\varphi^{\prime}}\left(\langle d(x, \cdot)\rangle_{\Gamma}^{\varphi}\right)\left[\varphi \circ\langle d(x, \cdot)\rangle_{\Gamma}^{\varphi}-\varphi \circ d(x, y)\right] d \Gamma_{0}(x)\right. \\
& +\left|\Gamma_{0}\right| \eta \kappa(p)\left[\psi\left(\left\langle\langle d(\cdot, \cdot)\rangle_{\Gamma_{0}}^{\varphi}\right\rangle_{\Gamma}^{\psi}\right)-\psi\left(\langle d(\cdot, y)\rangle_{\Gamma_{0}}^{\varphi}\right)\right]+\int_{\Gamma_{0}} \frac{\varphi^{\prime} \circ d(x, y)}{d(x, y)} \\
& \left.\times\left[v \frac{\psi^{\prime}}{\varphi^{\prime}}\left(\langle d(x, \cdot)\rangle_{\Gamma}^{\varphi}\right)+\eta \frac{\psi^{\prime}}{\varphi^{\prime}}\left(\langle d(\cdot, y)\rangle_{\Gamma_{0}}^{\varphi}\right)\right](y-x) \cdot \mathbf{n}(p) d \Gamma_{0}(x)\right] .
\end{aligned}
$$

We should note that all these results hold independently of the fact that the curves are open or closed since we only used in the integration by parts the fact that the field $\boldsymbol{\beta}$ was parallel to the normal field $\mathbf{n}$.

\section{Appendix B. Computation of $\nabla \tilde{\rho}_{D}\left(\Gamma, \Gamma_{0}\right)$}

We prove Proposition 27.

Proof. From the definitions

$$
\mathcal{G}\left(\tilde{\rho}_{D}\left(\Gamma, \Gamma_{0}\right), \boldsymbol{\beta}\right)=\mathcal{G}\left(\left\|\tilde{d}_{\Gamma}-\tilde{d}_{\Gamma_{0}}\right\|_{L^{2}(D)}, \boldsymbol{\beta}\right)+\mathcal{G}\left(\left\|\nabla\left(\tilde{d}_{\Gamma}-\tilde{d}_{\Gamma_{0}}\right)\right\|_{\mathbf{L}^{2}(D)}, \boldsymbol{\beta}\right),
$$


and

$\mathcal{G}\left(\left\|\tilde{d}_{\Gamma}-\tilde{d}_{\Gamma_{0}}\right\|_{L^{2}(D)}, \boldsymbol{\beta}\right)=\frac{1}{\left\|\tilde{d}_{\Gamma}-\tilde{d}_{\Gamma_{0}}\right\|_{L^{2}(D)}} \int_{D}\left|\tilde{d}_{\Gamma}(x)-\tilde{d}_{\Gamma_{0}}(x)\right| \mathcal{G}\left(\tilde{d}_{\Gamma}(x), \boldsymbol{\beta}\right) d x$,

and

$$
\begin{aligned}
\mathcal{G}\left(\left\|\nabla\left(\tilde{d}_{\Gamma}-\tilde{d}_{\Gamma_{0}}\right)\right\|_{\mathbf{L}^{2}(D)}, \boldsymbol{\beta}\right)= & \frac{1}{\left\|\nabla\left(\tilde{d}_{\Gamma}-\tilde{d}_{\Gamma_{0}}\right)\right\|_{\mathbf{L}^{2}(D)}} \\
& \times \int_{D} \nabla\left(\tilde{d}_{\Gamma}(x)-\tilde{d}_{\Gamma_{0}}(x)\right) \cdot \nabla\left(\mathcal{G}\left(\tilde{d}_{\Gamma}(x), \boldsymbol{\beta}\right)\right) d x .
\end{aligned}
$$

We now compute $\mathcal{G}\left(\tilde{d}_{\Gamma}(x), \boldsymbol{\beta}\right)$ and its gradient. Starting with (16), we readily obtain

$$
\begin{aligned}
\mathcal{G}\left(\tilde{d}_{\Gamma}(x), \boldsymbol{\beta}\right)=\frac{1}{|\Gamma| \varphi^{\prime}\left(\tilde{d}_{\Gamma}(x)\right)}[ & \left(\int_{\Gamma} \kappa(y) \mathbf{n}(y) \cdot \boldsymbol{\beta}(y) d \Gamma(y)\right)\langle\varphi \circ d(x, \cdot)\rangle_{\Gamma} \\
& +\int_{\Gamma} \varphi^{\prime}(d(x, y)) \frac{y-x}{d(x, y)} \cdot \boldsymbol{\beta}(y) d \Gamma(y) \\
& \left.-\int_{\Gamma} \varphi(d(x, y)) \kappa(y) \mathbf{n}(y) \cdot \boldsymbol{\beta}(y) d \Gamma(y)\right] .
\end{aligned}
$$

According to our initial hypothesis, $\boldsymbol{\beta}(y)=\beta(y) \mathbf{n}(y)$. We define

$$
B(x, y)=\kappa(y)\left(\langle\varphi \circ d(x, \cdot)\rangle_{\Gamma}-\varphi \circ d(x, y)\right)+\varphi^{\prime}(d(x, y)) \frac{y-x}{d(x, y)} \cdot \mathbf{n}(y),
$$

so that

$$
\mathcal{G}\left(\tilde{d}_{\Gamma}(x), \boldsymbol{\beta}\right)=\frac{1}{|\Gamma| \varphi^{\prime}\left(\tilde{d}_{\Gamma}(x)\right)} \int_{\Gamma} B(x, y) \beta(y) d \Gamma(y) .
$$

Let us compute the gradient of this expression with respect to the $x$ variable:

$$
\begin{aligned}
\nabla \mathcal{G}\left(\tilde{d}_{\Gamma}(x), \boldsymbol{\beta}\right)= & -\frac{\varphi^{\prime \prime}\left(\tilde{d}_{\Gamma}(x)\right)}{|\Gamma| \varphi^{\prime 2}\left(\tilde{d}_{\Gamma}(x)\right)}\left(\int_{\Gamma} B(x, y) \beta(y) d \Gamma(y)\right) \nabla \tilde{d}_{\Gamma}(x) \\
& +\frac{1}{|\Gamma| \varphi^{\prime}\left(\tilde{d}_{\Gamma}(x)\right)} \int_{\Gamma} \nabla B(x, y) \beta(y) d \Gamma(y) .
\end{aligned}
$$

After some manipulation, we find that

$$
\begin{aligned}
\nabla B(x, y)= & \kappa(y)\left(\left\langle\varphi^{\prime} \circ d(x, \cdot) \frac{x-\cdot}{d(x, \cdot)}\right\rangle_{\Gamma}-\varphi^{\prime} \circ d(x, y) \frac{x-y}{d(x, y)}\right) \\
& +\left(\varphi^{\prime \prime}(d(x, y))-\frac{\varphi^{\prime}(d(x, y)}{d(x, y)}\right) \mathbf{n}(y),
\end{aligned}
$$


where we have used, in particular, the fact that

$$
\nabla \tilde{d}_{\Gamma}(x)=\frac{1}{\varphi^{\prime}\left(\tilde{d}_{\Gamma}(x)\right)}\left\langle\varphi^{\prime} \circ d(x, \cdot) \frac{x-\cdot}{d(x, \cdot)}\right\rangle_{\Gamma} .
$$

We also define

$$
C_{1}(x)=\frac{1}{|\Gamma| \varphi^{\prime}\left(\tilde{d}_{\Gamma}(x)\right)}\left\|\tilde{d}_{\Gamma}-\tilde{d}_{\Gamma_{0}}\right\|_{L^{2}(D)}^{-1}\left(\tilde{d}_{\Gamma}(x)-\tilde{d}_{\Gamma_{0}}(x)\right),
$$

so that

$$
\begin{aligned}
\mathcal{G}\left(\left\|\tilde{d}_{\Gamma}-\tilde{d}_{\Gamma_{0}}\right\|_{L^{2}(D)}, \boldsymbol{\beta}\right) & =\int_{D} \int_{\Gamma} B(x, y) C_{1}(x) \beta(y) d \Gamma(y) d x \\
& =\int_{\Gamma}\left(\int_{D} B(x, y) C_{1}(x) d x\right) \beta(y) d \Gamma(y) .
\end{aligned}
$$

We then define the vector quantity

$$
\mathbf{C}_{2}(x)=\frac{1}{|\Gamma| \varphi^{\prime}\left(\tilde{d}_{\Gamma}(x)\right)}\left\|\nabla\left(\tilde{d}_{\Gamma}-\tilde{d}_{\Gamma_{0}}\right)\right\|_{\mathbf{L}^{2}(D)}^{-1} \nabla\left(\tilde{d}_{\Gamma}-\tilde{d}_{\Gamma_{0}}\right)(x),
$$

so that

$$
\begin{aligned}
\mathcal{G}\left(\| \nabla\left(\tilde{d}_{\Gamma}-\right.\right. & \left.\left.\tilde{d}_{\Gamma_{0}}\right) \|_{\mathbf{L}^{2}(D)}, \boldsymbol{\beta}\right) \\
= & -\int_{D} \int_{\Gamma} \frac{\varphi^{\prime \prime}}{\varphi^{\prime}}\left(\tilde{d}_{\Gamma}(x)\right)\left(\mathbf{C}_{2}(x) \cdot \nabla \tilde{d}_{\Gamma}(x)\right) B(x, y) \beta(y) d \Gamma(y) d x \\
& +\int_{D} \int_{\Gamma}\left(\mathbf{C}_{2}(x) \cdot \nabla B(x, y)\right) \beta(y) d \Gamma(y) d x \\
= & \int_{\Gamma}\left(\int _ { D } \left(\mathbf{C}_{2}(x) \cdot \nabla B(x, y)-\frac{\varphi^{\prime \prime}}{\varphi^{\prime}}\left(\tilde{d}_{\Gamma}(x)\right)\left(\mathbf{C}_{2}(x) \cdot \nabla d(x, \Gamma)\right)\right.\right. \\
& \times B(x, y)) d x) \beta(y) d \Gamma(y) .
\end{aligned}
$$

Combining equations (43) and (44) we obtain the corresponding gradient $\nabla \tilde{\rho}_{D}$ $\left(\Gamma, \Gamma_{0}\right)$ :

$$
\begin{aligned}
& \nabla \tilde{\rho}_{D}\left(\Gamma, \Gamma_{0}\right)(y) \\
= & \int_{D}\left[B(x, y)\left(C_{1}(x)-\frac{\varphi^{\prime \prime}}{\varphi^{\prime}}\left(\tilde{d}_{\Gamma}(x)\right)\left(\mathbf{C}_{2}(x) \cdot \nabla \tilde{d}_{\Gamma}(x)\right)\right)+\mathbf{C}_{2}(x) \cdot \nabla B(x, y)\right] d x,
\end{aligned}
$$

and this completes the proof.

\section{References}

[1] D. Adalsteinsson and J. A. Sethian, The fast construction of extension velocities in level set methods, J. Comput. Phys. 148(1) (1999), 2-22. 
[2] L. Ambrosio and H. M. Soner, Level set approach to mean curvature flow in arbitrary codimension, J. Differential Geom. 43 (1996), 693-737.

[3] A. Blake and M. Isard, Active Contours, Springer-Verlag, New York, 1998.

[4] F. L. Bookstein, Size and shape spaces for landmark data in two dimensions, Statist. Sci. 1 (1986), $181-242$.

[5] T. K. Carne, The geometry of shape spaces, Proc. London Math. Soc. 3(61) (1990), 407-432.

[6] Hyeong In Choi, Sung Woo Choi, and Hwan Pyo Moon, Mathematical theory of the medial axis transform, Pacific J. Math. 181 (1997), 57-88.

[7] G. Choquet, Cours d'Analyse, Vol. II, Masson, Paris, 1969.

[8] T. Cootes, C. Taylor, D. Cooper, and J. Graham, Active shape models-Their training and application, Comput. Vision Image Understanding 61(1) (1995), 38-59.

[9] M. C. Delfour and J.-P. Zolésio, Shape analysis via oriented distance functions, J. Funct. Anal. 123(1) (1994), 129-201.

[10] M. C. Delfour and J.-P. Zolésio, Shape analysis via distance functions: Local theory, in Boundaries, Interfaces and Transitions, CRM Proc. Lecture Notes, Vol. 13, pp. 91-123, American Mathematical Society, Providence, RI, 1998.

[11] M. C. Delfour and J.-P. Zolésio, Shapes and Geometries, Advances in Design and Control, SIAM, Philadelphia, PA, 2001.

[12] J. Dieudonné, Éléments d'Analyse, Vol. I, Gauthier-Villars, Paris, 1969.

[13] I. L. Dryden and K. V. Mardia, Statistical Shape Analysis, Wiley, New York, 1998.

[14] P. Dupuis, U. Grenander, and M. Miller, Variational problems on flows of diffeomorphisms for image matching, Quart. Appl. Math. 56 (1998), 567-600.

[15] L. C. Evans, Partial Differential Equations, Graduate Studies in Mathematics, Vol. 19, Proc. Amer. Math. Soc. (1998).

[16] H. Federer, Hausdorff measure and Lebesgue area, Proc. Nat. Acad. Sci. USA 37 (1951), 90-94.

[17] M. Fréchet, L'intégrale abstraite d'une fonction abstraite d'une variable abstraite et son application à la moyenne d'un Élément aléatoire de nature quelconque, Rev. Scientifique 82 (1944), 483-512.

[18] M. Fréchet, Les éléments aléatoires de nature quelconque dans un espace distancié, Ann. Inst. H. Poincaré 10 (1948), 215-310.

[19] M. Fréchet, Les courbes aléatoires, Bull. Inst. Internat. Statist. 38 (1961), 499-504.

[20] J. Gomes and O. Faugeras, Reconciling distance functions and level sets, J. Visual Commun. Image Representation 11 (2000), 209-223.

[21] U. Grenander, General Pattern Theory, Oxford University Press, Oxford, UK, 1993.

[22] U. Grenander, Y. Chow, and D. Keenan, HANDS: A Pattern Theoretic Study of Biological Shapes, Springer-Verlag, New York, 1990.

[23] U. Grenander and M. Miller, Computational anatomy: An emerging discipline, Quart. Appl. Math. 56(4) (1998), 617-694.

[24] E. G. Harding and D. G. Kendall (eds.), Foundation of a theory of random sets, in Stochastic Geometry, pp. 322-376, Wiley, New York, 1973.

[25] H. Karcher, Riemannian centre of mass and mollifier smoothing, Comm. Pure Appl. Math. 30 (1977), 509-541.

[26] D. G. Kendall, Shape manifolds, procrustean metrics and complex projective spaces, Bull. London Math. Soc. 16 (1984), 81-121.

[27] D. G. Kendall, A survey of the statistical theory of shape, Statist. Sci. 4(2) (1989), 87-120.

[28] W. Kendall, Probability, convexity, and harmonic maps with small image $i$ : Uniqueness and fine existence, Proc. London Math. Soc. 61(2) (1990), 371-406.

[29] E. Klassen, A. Srivastava, W. Mio, and S. H. Joshi, Analysis of planar shapes using geodesic paths on shape spaces, IEEE Trans. Pattern Anal. Machine Intell. 26(3) (2004), 372-383.

[30] M. Leventon, E. Grimson, and O. Faugeras, Statistical shape influence in geodesic active contours, in Proceedings of the International Conference on Computer Vision and Pattern Recognition, pp. 316-323, Hilton Head Island, South Carolina, June 2000, IEEE Computer Society, New York.

[31] M. Leventon, Anatomical Shape Models for Medical Image Analysis, PhD thesis, MIT, 2000.

[32] W. E. Lorensen and H. E. Cline, Marching cubes: A high resolution 3d surface construction 
algorithm, in Proceedings of the SIGGRAPH, M. C. Stone (ed.), pp. 163-169, Anaheim, CA, July 1987, in Computer Graphics, Vol. 21, No. 4.

[33] G. Matheron, Random Sets and Integral Geometry, Wiley, New York, 1975.

[34] M. Miller and L. Younes, Group actions, homeomorphisms, and matching: A general framework, Internat. J. Comput. Vision 41(1/2) (2001), 61-84.

[35] S. Osher and N. Paragios (eds.), Geometric Level Set Methods in Imaging, Vision and Graphics, Springer-Verlag, New York, 2003.

[36] S. Osher and J. Sethian, Fronts propagating with curvature dependent speed: Algorithms based on the Hamilton-Jacobi formulation, J. Comput. Phys. 79 (1988), 12-49.

[37] D. Peng, B. Merriman, S. Osher, H. Zhao, and M. Kang, A PDE-based fast local level set method, J. Comput. Phys. 155(2) (1999), 410-438.

[38] X. Pennec, L'Incertitude dans les Problèmes de Reconnaissance et de Recalage-Applications en Imagerie Médicale et Biologie Moléculaire, $\mathrm{PhD}$ thesis, Ecole Polytechnique, Palaiseau (France), December 1996.

[39] W. Rudin, Real and Complex Analysis, McGraw-Hill, New York, 1966.

[40] J. Serra, Image Analysis and Mathematical Morphology, Academic Press, London, 1982.

[41] J. A. Sethian, Level Set Methods and Fast Marching Methods: Evolving Interfaces in Computational Geometry, Fluid Mechanics, Computer Vision, and Materials Sciences. Cambridge Monograph on Applied and Computational Mathematics, Cambridge University Press, New York, 1999.

[42] C. G. Small, The Statistical Theory of Shapes, Springer-Verlag, New York, 1996.

[43] S. Soatto and A. J. Yezzi, DEFORMOTION, deforming motion, shape average and the joint registration and segmentation of images, in Proceedings of the 7th European Conference on Computer Vision, A. Heyden, G. Sparr, M. Nielsen, and P. Johansen (eds.), pp. 32-47, Copenhagen, Denmark, May 2002, Springer-Verlag, New York.

[44] M. Sussman, P. Smereka, and S. Osher, A level set approach for computing solutions to incompressible two-phase flow, J. Comput. Phys. 114 (1994), 146-159.

[45] A. Toga and P. Thompson, The role of image registration in brain mapping, Image and Vision Comput. 19(1-2) (2001), 3-24.

[46] A. Toga (ed.), Brain Warping, Academic Press, New York, 1998.

[47] A. Trouvé and L. Younes, Diffeomorphic matching problems in one dimension: Designing and minimizing matching functionals, in Proceedings of the 6th European Conference on Computer Vision, pp. 573-587, Dublin, Ireland, June 2000.

[48] A. Trouvé and L. Younes, Mise en correspondance par difféomorphismes en une dimension: Définition et maximisation de fonctionnelles, in 12ème Congrès RFIA '00, Paris, February 2000.

[49] A. Trouvé, Diffeomorphism groups and pattern matching in image analysis, Internat. J. Comput. Vision 28(3) (1998), 213-221.

[50] L. Younes, Computable elastic distances between shapes, SIAM J. Appl. Math. 58(2) (1998), $565-586$.

[51] L. Younes, Optimal matching between shapes via elastic deformations, Image and Vision Comput. 17(5/6) (1999), 381-389.

[52] L. Younes, Invariance, déformations et reconnaissance de formes, Mathematiques and Applications, Springer-Verlag, New York, 2003. 